\title{
Molecular ultrasound imaging
}

Citation for published version (APA):

Baleanu-Curaj, A. (2017). Molecular ultrasound imaging: a powerful tool for the diagnosis of endothelial dysfunction and arterial inflammation. [Doctoral Thesis, Maastricht University]. Maastricht University. https://doi.org/10.26481/dis.20171102abc

Document status and date:

Published: 01/01/2017

DOI:

10.26481/dis.20171102abc

Document Version:

Publisher's PDF, also known as Version of record

\section{Please check the document version of this publication:}

- A submitted manuscript is the version of the article upon submission and before peer-review. There can be important differences between the submitted version and the official published version of record.

People interested in the research are advised to contact the author for the final version of the publication, or visit the DOI to the publisher's website.

- The final author version and the galley proof are versions of the publication after peer review.

- The final published version features the final layout of the paper including the volume, issue and page numbers.

Link to publication

\footnotetext{
General rights rights.

- You may freely distribute the URL identifying the publication in the public portal. please follow below link for the End User Agreement:

www.umlib.nl/taverne-license

Take down policy

If you believe that this document breaches copyright please contact us at:

repository@maastrichtuniversity.nl

providing details and we will investigate your claim.
}

Copyright and moral rights for the publications made accessible in the public portal are retained by the authors and/or other copyright owners and it is a condition of accessing publications that users recognise and abide by the legal requirements associated with these

- Users may download and print one copy of any publication from the public portal for the purpose of private study or research.

- You may not further distribute the material or use it for any profit-making activity or commercial gain

If the publication is distributed under the terms of Article $25 \mathrm{fa}$ of the Dutch Copyright Act, indicated by the "Taverne" license above, 
Molecular ultrasound imaging - a powerful tool for the diagnosis of endothelial dysfunction and arterial inflammation

\section{Adelina Băleanu-Curaj}


(C) 2017 Adelina Băleanu-Curaj

ISBN: 978-3-00-056572-4

Printed by: TOP SERV R-98 SRL

Cover design: Dr. Cosmin Holeab

(Cover illustration was purchased from Shutterstock and depicts liquid droplets and waves resembling the microbubbles and the ultrasound waves, respectively.) 
"We do not remember days, we remember moments."

- Caesar Pavese 



\section{Molecular ultrasound imaging - a powerful tool for the diagnosis of endothelial dysfunction and arterial inflammation}

\section{DISSERTATION}

To obtain the degree of Doctor at Maastricht University, on the authority of the Rector Magnificus, Prof. Dr. Rianne M. Letschert, in accordance with the decision of the Board of Deans, to be defended in public on

Thursday $2^{\text {nd }}$ November 2017 , at 16:00 hours

by

Adelina Băleanu-Curaj 
Supervisors:

Prof. Dr. F.C.S. Ramaekers

Prof. Dr. M.A.M.J. van Zandvoort

Prof. Dr. F. Kießling (University of Aachen)

Assessment Committee:

Prof. Dr. W.H. Backes (Chairman)

Dr. K. Reesink

Prof. Dr. J.K. Willmann (University of Stanford)

Prof. Dr. C.T.W. Moonen (University of Utrecht) 


\section{Contents}

Chapter 1 General introduction

Chapter 2 Rhodamine-loaded intercellular adhesion

molecule-1-targeted microbubbles for dualmodality imaging under controlled shear stresses.

Chapter 3 Induction of accelerated atherosclerosis in mice: the "wire-injury" model.

Chapter 4 Noninvasive molecular ultrasound monitoring of vessel healing after intravascular surgical procedures in a preclinical setup.

Chapter 5 Molecular ultrasound imaging of JAM-A depicts acute alterations in blood flow and early endothelial dysregulation

Chapter 6 General discussion

Summary

Valorization addendum

List of abbreviations

Biography

List of publications

Acknowledgements 



\section{Chapter 1}

General introduction 


\section{The anatomy and physiology of carotid arteries}

The two common carotid arteries, right and left carotid artery, are major blood vessels that supply blood to head and neck. They differ in origin, length, and anatomical relationship. The right carotid originates from the brachiocephalic trunk and is a little longer than the contralateral artery, while the left carotid springs directly from the aortic arch. At the neck region, both left and right carotid artery divide into two branches:

- $\quad$ The internal carotid artery, which supplies blood to the brain;

- The external carotid artery, which supplies blood to face and neck;

Between the two common carotid arteries there is free communication, allowing collateral circulation development in case of slow-progressive obstruction of one common carotid artery.

The carotid arteries are made of three layers of tissue (see Figure $1 \mathrm{~A}$ ):

- Tunica intima: the innermost layer in direct contact with blood flow, made up of:

- One layer of endothelial cells (EC), covered on the luminal side with a continuous layer of proteoglycans, the endothelial glycocalyx ${ }^{1}$;

- A thin subendothelial layer of connective tissue containing collagen type I and II and proteoglycans;

- Internal elastic lamina: consists of a membrane containing a network of elastic fibers, arranged longitudinally.

- Tunica media: between the tunica intima, on the inside, and the tunica externa, on the outside, consisting of:

- Elastic fibers;

- Smooth muscle cells (SMC).

Depending on the size of the artery, the SMC are arranged in one layer (in small arteries) or multiple layers (up to 7, in large arteries). Also, the amount of elastic fibers is increasing with the size of the artery. 
- Tunica externa (adventitia): the outermost layer of a blood vessel, providing a limiting barrier that protects the vessel from stretching beyond itsphysiological limit during systole. It consists of:

- Fibroblasts;

- External elastic lamina;

- Collagen fibers.

Also, characteristic of this layer is the presence of small blood vessels called the vasa vasorum that supply the walls of larger arteries, and nerve fibers.

As the arteries are further away from the heart, the arterial constitution changes, getting adapted to their local functions. The walls of arteries close to the heart (as the carotid artery) are thick and very elastic in order to withstand pulsatile flow and blood pressure. As arteries become smaller their wall thickness gradually decreases and their composition changes. Indeed, they become less elastic and contain more SMC.

\section{Endothelial dysfunction, inflammation and atherosclerosis}

Atherosclerosis, which is a chronic inflammatory disease developing in the arterial vessels, is the most common cardiovascular disease. It is a multifactorial and multistep disease that involves chronic inflammation at every stage ${ }^{2}$.

The endothelium is the inner layer of blood vessel wall in contact with the blood flow, through on organized glycocalyx layer. The glycocalyx is an important determinant of vascular permeability, being able to limit the access of certain molecules to the endothelial cell membrane ${ }^{1}$. Although only one cell layer thick, the endothelium serves important homeostatic functions. It can detect and react to changes in hemodynamic forces or, to chemical and mechanical stimuli by synthesis or release of a wide range of factors regulating vascular tone, cellular recruitment, thromboresistance, SMC proliferation, and local inflammation. 
Endothelial activation/dysfunction represents a switch from a dormant towards proinflammatory and procoagulant phenotype ${ }^{3}$. On activation, the endothelium expresses chemokines, cytokines, integrins, and adhesion molecules, harbored in the disorganized endothelial glycocalyx ${ }^{1}$ (Figure 1C). These molecules are designed to interact with leukocytes and platelets. Thus, activated/dysfunctional endothelium plays a dual role in hemostasis and inflammation.

Endothelial dysfunction accompanied by the upregulation of selectins (P-, E- and LSelectin), adhesion molecules (intercellular (ICAM-1) and vascular cell (VCAM-1) adhesion molecules), and integrins ${ }^{4}$ is triggered by multiple factors, such as hypercholesterolemia, hyperglycemia, insulin resistance, hypertension, and ageing. Particularly, endothelial dysfunction with glycocalyx disorganization and hypercholesterolemia are the cause of atherosclerotic plaque development.

Atherosclerosis usually develops in areas of low $\left(<6 \mathrm{din} / \mathrm{cm}^{2}\right)$, oscillating, or retrograde shear stress. Indeed, at these sites, NO release is diminished and adhesion molecules, chemokines, and growth factors are increased, together creating a pro-inflammatory environment. Herein, selectins and their ligands are involved in trapping and rolling of circulating inflammatory cells which accumulate in the early atherosclerotic plaque, the monocytes and T lymphocytes ${ }^{5,6}$, and promote the onset of atherosclerosis ${ }^{7}$. The interaction of leukocytes with the EC is mediated by the glycocalyx, which seems to have a dual role. On the one hand, the glycocalyx hosts the EC adhesion molecules and on the other hand, it attenuates adhesion of leukocytes to these molecules ${ }^{1}$. Adhesion molecules, such as ICAM-1 and VCAM1 , and integrins induce firm adhesion of inflammatory cells at the vascular surface, while platelet endothelial cellular adhesion molecules (PECAM-1) and junctional adhesion molecule (JAM) -A are involved in transmigration of inflammatory cells from the blood compartment into the vessel wall ${ }^{8}$, across the EC. Once resident in the arterial intima, monocytes mature into unpolarized macrophages (M0) under the presence of macrophage-colony stimulating factor (M-CSF) ${ }^{9}$. Further, the $\mathrm{M} 0$ under the stimulation of IFN- $\gamma$, LPS, immune complexes, M-CSF, interleukins, TGF- $\beta$, glucocorticoids, or platelet factor 4 polarize into M1, M2, M4 or Mox ${ }^{9,10}$.

Prior to a mature atherosclerotic plaque, a so-called "nonatherosclerotic intimal 
lesion" develops, consisting of intimal thickening due to SMC migration to the subendothelial layer ${ }^{11}$ and their proteoglycans ${ }^{12}$ production.

A

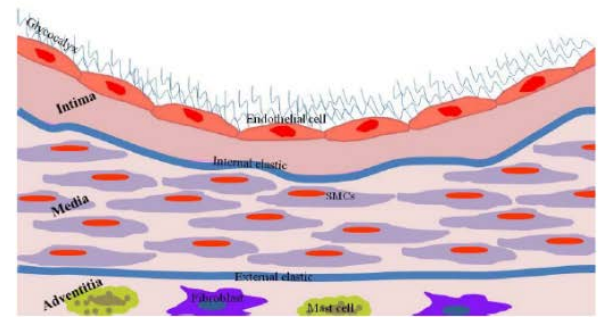

B

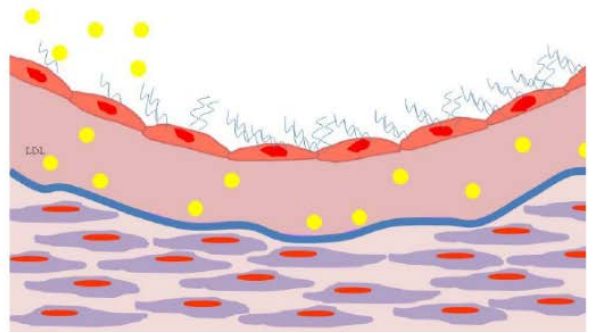

C

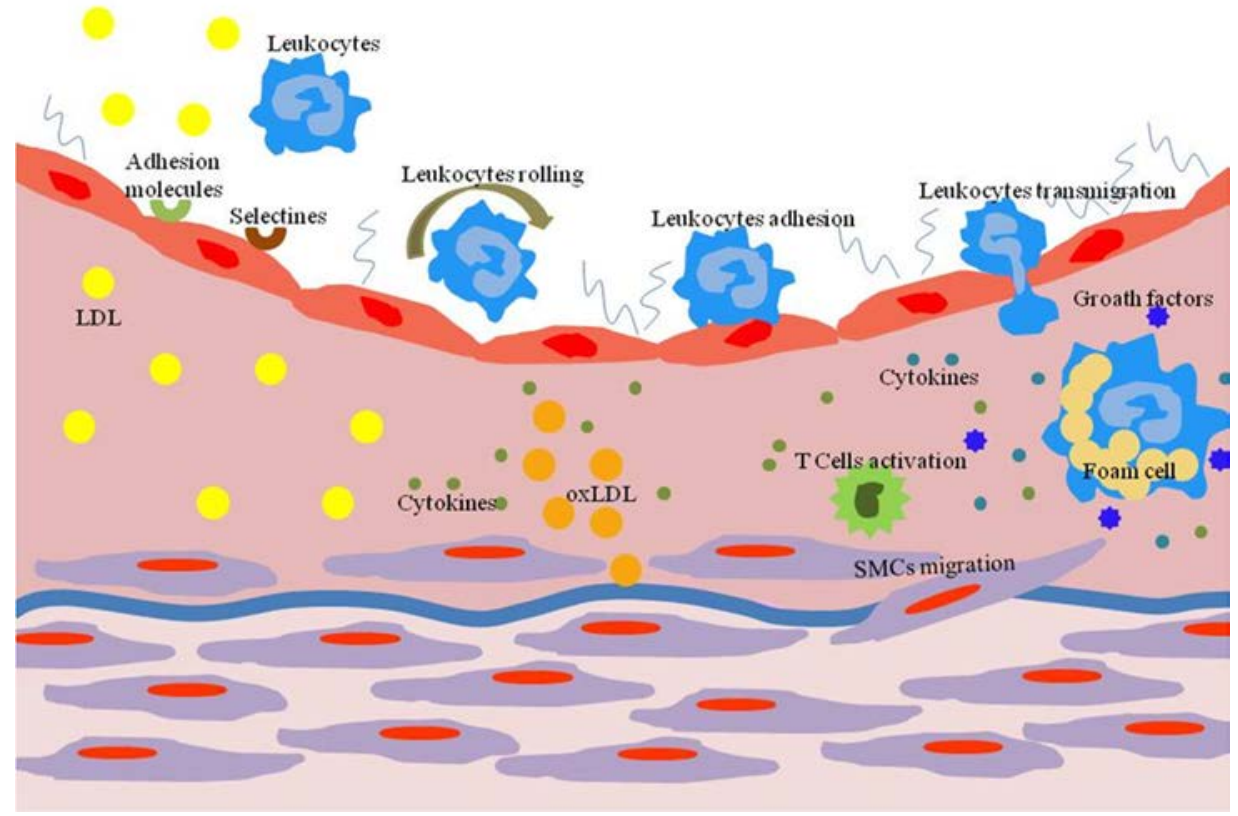

Figure 1: Atherosclerotic plaque formation. (A) A healthy artery. (B). The Endothelial glycocalyx is reduced and disorganized. Hyperlipidemia determines the subendothelial retention of LDL with intimal layer expansion. (C) Accumulated LDL within the intima undergoes oxidative modification and further supports local inflammation. Various adhesion molecules (selectins, adhesion molecules) are expressed on the luminal side of the activated EC. Therefore, circulating leukocytes are recruited and transmigrate to the subendothelial space. Here, monocytes transform into macrophages to remove the accumulated LDL. Lipoprotein uptake by macrophages results in foam cell formation. Reprinted from ${ }^{13}$. 
Concomitantly, low density lipoproteins (LDL) are passing through the dysfunctional endothelial layer and accumulate in the subendothelial space (Figure 1B). Here, the proteoglycans facilitate LDL particles oxidative modification ${ }^{14}$ and thus promote an inflammatory response at sites of lesion formation ${ }^{11,14,15}$. In the plaque, the mononuclear phagocytes type $\mathrm{M} 2$ express scavenger receptors necessary for the uptake of modified lipoproteins and, hence, form foam cells ${ }^{16}$ (Figure 1).

In advanced plaques, lipid loaded macrophages accumulate and finally undergo apoptosis, contributing centrally to the formation of plaque necrosis. The further expansion of the necrotic core along with the thinning of the fibrous cap underlies the instability that promotes plaque rupture.

Therefore, at later stages, high shear stresses $\left(>70 \mathrm{din} / \mathrm{cm}^{2}\right)$ can cause endothelial erosion, plaque rupture, and platelet aggregation ${ }^{17-19}$ with vessel obstruction. Thus, shear stress explains the importance of the haemodynamic factor in both plaque formation and rupture.

\section{Atherosclerosis treatment}

The therapy of atherosclerosis can be classified into chemical and interventional.

1) The chemical therapy consists in lipid lowering and anti-inflammatory compounds administrated as long term treatment of moderate atherosclerosis, with the therapeutical target of plaque regression.

2) The interventional therapy is applied in case of symptomatic or complicated atherosclerotic plaques and consists of balloon angioplasty with/without stent implantation, endarterectomy or bypass surgery. Except for the bypass surgery, the other interventional therapies involve endothelial damage naturally followed by neointima formation. To avoid acute thrombosis due to endothelial damage, dual antiplatelet therapy is prescribed for at least one year after the intervention. Meanwhile, EC proliferate until the monolayer is fully rebuilt and regain its role as a barrier. 


\section{Ultrasound imaging}

\section{Principles}

Medical ultrasound (US) is a diagnostic imaging technique that uses high frequency sound waves and their echoes to see internal body structures. US is currently one of the most frequently used and versatile imaging modality in medicine ${ }^{20}$. It has certain advantages by being cheap, broadly available, easy to handle, non-invasive, bed-side available and radiation free real-time imaging modality.

Ultrasonic images or sonograms are made by sending high-frequency (1 to 40 $\mathrm{MHz}^{21}$ ) sound pulses into tissue using a probe (or transducer). A signal generated by an ultrasonic transducer typically consists of a pulse of a few $\mu$ s with a certain center frequency. As the US waves penetrate body tissues, some get scattered, some get absorbed (or attenuated) by the tissue, and some are reflected to the transducer which works as both emitter and receptor. By means of different acoustic impedances along the path of transmission, some US waves are reflected to the transducer (echo signals) when they hit boundaries between tissues, and some continue to penetrate deeper until they reach another boundary and get reflected. The reflected sound waves are captured by the probe and further analyzed by the computer incorporated into the US device. The computer calculates the distance from the probe to the tissue or organ (boundaries) using the speed of sound in tissue and displays the distances and intensities of the echoes on the screen. As US transverses tissue, its energy decreases. This is called attenuation and is more pronounced in tissue with less density, such as the lungs.

Modern US transducers contain multiple piezoelectric crystals which are interconnected and vibrate in response to an applied electric current, converting electrical energy into US waves, and vice-versa, applied mechanical vibrations induce electrical signals ${ }^{22,23}$.

Sound waves can be described in terms of their frequency, wavelength, and amplitude: 
- Frequency is defined by the number of cycles that occur per unit time. The units of frequency are cycles/sec or Hertz $(\mathrm{Hz})$ (Figure 2).

- Wavelength is defined as the length of a single cycle. Typical values of wavelength are $0.1-0.8 \mathrm{~mm}$. It influences the longitudinal image resolution.

- Amplitude is defined as the difference between the peak value and the average value of the waveform. It is expressed in decibels $(\mathrm{dB})$. It is a parameter than can be changed by the sonographer. Amplitude decreases by $1 \mathrm{~dB} / 1 \mathrm{MHz} / 1$ centimeter traveled, as the US penetrates through tissue.

An important aspect is that higher frequencies of US, which have shorter wavelengths, are absorbed/attenuated more easily. Consequently, higher frequencies are not as penetrating and therefore used for the superficial body structures, and low frequencies are used for those that are deeper.

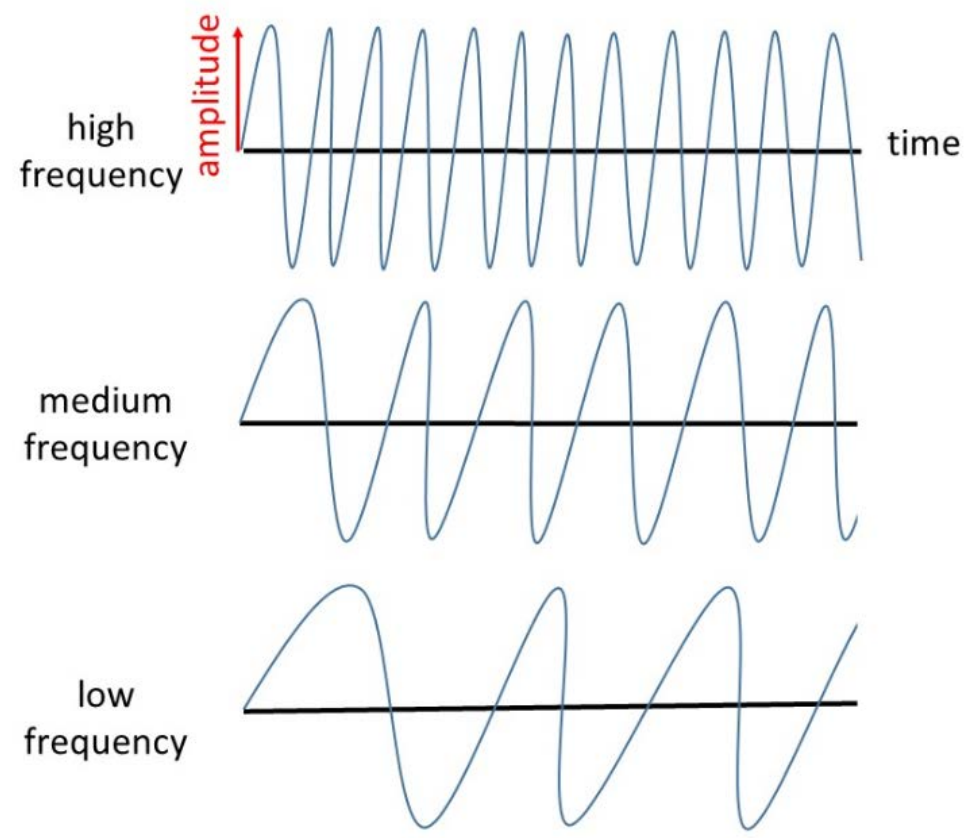

Figure 2. Schematic representation of US pulse frequencies. High frequencies are used for superficial body structures imaging, and low frequencies are suitable for deep tissue imaging. 


\section{Functional and morphological ultrasound}

US imaging has a broad spectrum of application in both medical research field and clinical practice, from functional brain imaging ${ }^{24}$ and fetal sonography, to blood vessels and parenchymal internal structures as liver, kidney, ovary and heart imaging.

Sonography is not only performed by radiologists, but as well by anesthetists to perform US guided anesthetic injections close to nerves. Gastroenterologists use it for imaging the liver, pancreas, spleen and when inflamed as well the appendix. Gynecologists use US to check on the development of the fetus, or to image the ovary and the uterus. Urologists image the testicles, prostate, kidney and bladder. In the cardiovascular field, diagnostic US is applied for heart ${ }^{25}$ and vessels imaging $^{26}$, providing significant insight into the physiology and pathology. In clinical practice, the left heart, the carotid and femoral arteries imaging are included in the routine diagnostic protocols. Lately, the right heart imaging has gained attention as well ${ }^{27}$.

There are several basic modalities to display the data:

- B-mode: B stands for brightness. The amplitude signal is converted into brightness. The brightness of each point is determined by the amplitude of the returned signal. The higher the amplitude, the brighter the dot is. It is the most used modality for orientation in the body, and for localizing internal structures.

- M-mode: $M$ stands for motion. It provides a 2D representation of anatomical structures along a single US beam in motion. It is generally used in cardiovascular research to study the movement of the vessel walls, myocardium, and to quantify cavity dimensions.

- Doppler mode: it makes use of the frequency shift due to relative motion between two object. Therefore, information regarding the blood velocity and cardiac valves can be obtained.

- Pulse Wave Doppler (PWD): it is used to depict velocity and direction of 
flow. The PWD also gives the user information about the nature of flow, -laminar or turbulent. In laminar flows, because most of the red blood cells (RBC) are traveling at the same velocity, the Doppler waveform has a thick white edge and is black within. In contrast, turbulent flow determines a wide distribution of RBC velocities making the Doppler wave appear filled-in.

- Color Doppler: in this mode, the velocity and direction of blood flows are depicted in a color map superimposed on the 2-D image. It uses pulse wave Doppler signals to create specific images. By convention, blood flowing away from the transducer is color coded in blue and that flowing toward the transducer in red.

In atherosclerosis, US imaging is generally applied to evaluate the stenosis level of an artery and arterial stiffness, or to evaluate, the successful reopening of an obstructed artery.

\section{Molecular ultrasound}

Molecular US is an imaging approach that combines classical US technology with molecularly-targeted probes ${ }^{28}$. US contrast agents act as an intravascular tracer, being preferentially detected over surrounding tissue when excited with US (insonicated). This allows the visualization of perfusion in living tissues. Molecular US contrast agents comprise microbubbles $(\mathrm{MB})^{29-31}$, echogenic liposomes ${ }^{32}$, perfluorocarbon droplets ${ }^{33}$, and other materials like gold particles ${ }^{34}$, decorated with binding ligands such as antibodies or small peptides targeted to specific molecular markers involved in various disease processes ${ }^{28,35}$. Ligand-decorated US contrast agents can be produced either by incorporating the specific ligands during their synthesis, or by attaching the ligands to the preformed contrast agent. Following intravenous administration, these targeted contrast agents accumulate at tissue sites expressing specific molecular markers, and due to their density and compressibility substantially different from that of blood and tissue, the US imaging signal is enhanced. 
The most broadly used US contrast agents comprise gas-filled MB suspensions. MB have a diameter of 1-5 $\mu \mathrm{m}$ and thus are capable of going through small blood capillaries in the body, but stay strictly intravascular. MB consist in a shell and a gas core (Figure 3).

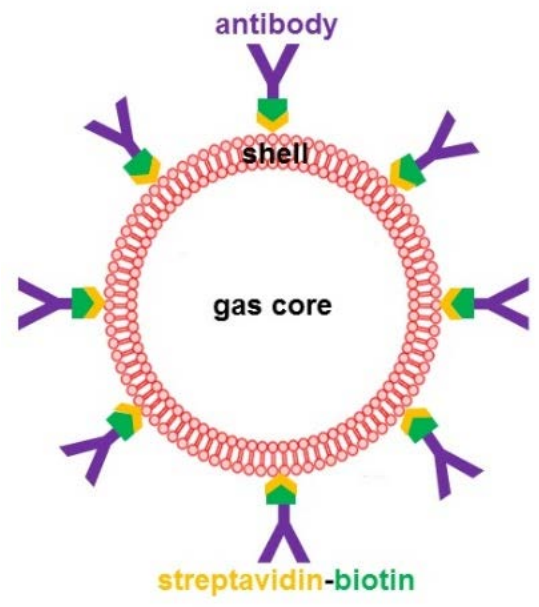

Figure 3. Microbubble composition. A microbubble consists in a shell, and a gas core. For functionalization, we attached antibodies to the MB shell using the streptavidin-biotin system.

The gas core comprises most of the particle volume and provides the mechanism for echogenicity. The gas core oscillates under the ultrasonic field making the MB expand during the rarefaction phase of the pressure wave and contract during the compression phase $^{36}$ (Figure 4). Gas cores can be composed of air, or high molecular weight gases such as perfluorocarbon, sulfur hexafluoride or nitrogen. MB with heavy gas cores have a longer lifetime because heavy gases are less watersoluble and- therefore it is less probable to leak out leading to MB dissolution.The MB shell prevents aggregation of the microbubbles and the gas leakage. The shell material determines as well the lifetime in circulation and the mechanical elasticity. The MB with a more hydrophilic shell tend to be taken up by the immune system more easily, and therefore the time available for contrast imaging is being shorten up. The shell may be composed of surfactants, lipids, proteins, polymers, or a mixture of these. The more elastic the material, the more acoustic energy it can withstand before bursting. Therefore, MB can be characterized as soft-shelled MB, more suitable for harmonic (nonlinear) imaging, and hard-shelled MB, more suitable 
for destructive US imaging procedures, such as Power Doppler US ${ }^{37}$. In the studies included in this thesis, air filled polymer-shell (poly(n-butyl cyanoacrylate)) MB were used.

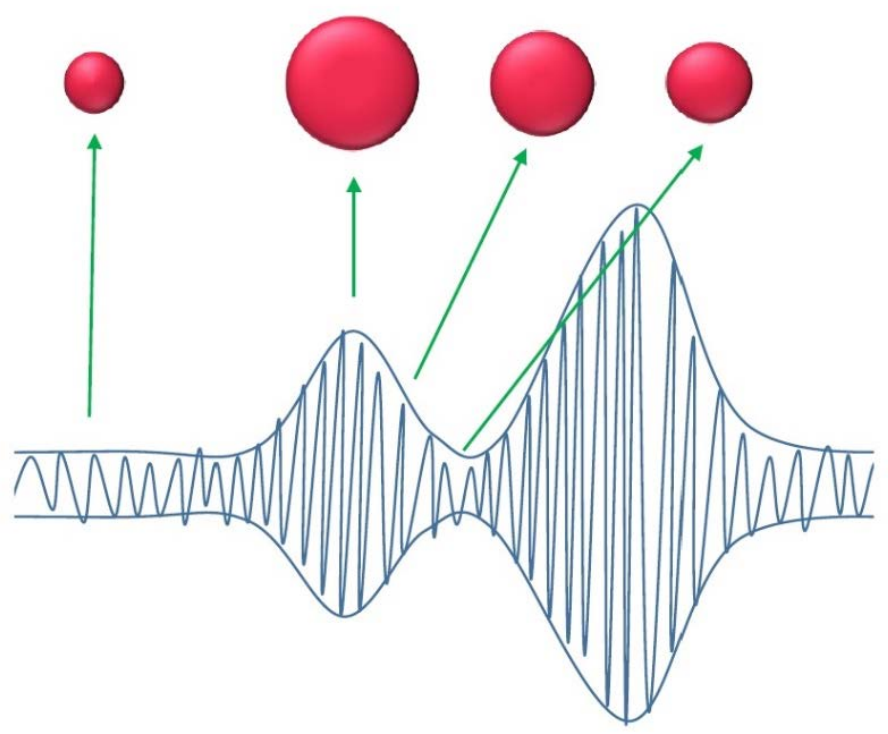

Figure 4. Oscillation of a microbubble in response to an US field. MB expands during the rarefaction phase of the pressure wave, and contracts during the compression.

Former preclinical high-frequency US devices, such as the Vevo770 from Visual Sonics, were using the Linear Contrast Mode for detecting the MB. This means that, in standard B-Mode imaging, the MB could be visualized as very bright dots due to their high echogenicity when insonicated with high-frequency US waves. To separate the signal generated by MB from the one generated by the tissue (process called segmentation), post-processing algorithms are used. However, in many cases the MB and tissue are comparably bright, resulting in poor segmentation possibility, and consequently a difficult visualization of the MB.

New generation US devices make use of the MB propriety to oscillate nonlinearly. A nonlinear oscillation occurs because the radial expansion of the MB differs from the radial contraction. On the one hand, due to their high shell elasticity properties, during the expansion phase of oscillation the radius of the gas MB can increase by 
several times its original size. On the other hand, during the contraction phase of the oscillation, the radius is limited since the gas molecules are forced closer together, making it harder to compress. This means that, the oscillation is asymmetrical, causing nonlinear scattering of US waves ${ }^{38}$. Therefore, the MB-generated signal can be discriminate from the tissue signal, when imaged at low acoustic pressure (below $\sim 500 \mathrm{kPa}$ ). When increasing the acoustic pressure, tissue will respond nonlinearly as well, causing some residual nonlinear background tissue signal ${ }^{28}$. Analyzing the frequency content of the nonlinear signal after interacting with a MB, harmonic components are recognized. Thereby, the backscattered waves consist of a multiple of the center frequency of the incident US wave, called harmonics ${ }^{39}$ (second, third or more), or half of the center frequency, named subharmonic ${ }^{40}$.

Most of the current preclinical and clinical US systems apply multiple pulses for nonlinear contrast agent detection. Two of the most used multi-pulse techniques are the pulse inversion (PI) and the amplitude modulation (AM), which form the basis of current nonlinear contrast agent detection. In both cases, the targeted result is a complete cancellation of the tissue signal, while a persistence of residual signal difference that can be exploited to detect MB is expected. Multi-pulse techniques also have the advantage of reduced noise in the image, since signal averaging in case of tissue is close to- or equal to zero.

Molecular US imaging is typically performed as follows: the transducer is fixed with a mechanical arm in position ready to image the region of interest. Fixation is necessary to avoid motion artifacts from operator movements. A suspension of targeted MB is administered into the peripheral vasculature (Figure 5), followed by a waiting periodof up to 30 minutes.

During this period, targeted MB accumulate intravascularly at the target level, followed by a second phase when freely circulating MB are cleared from the system. Imaging is then performed at low mechanical index to avoid unwanted MB destruction, followed by a destruction pulse which clears all contrast agent within the field of view, and then imaging again at low mechanical index for the rest of the imaging sequence. 


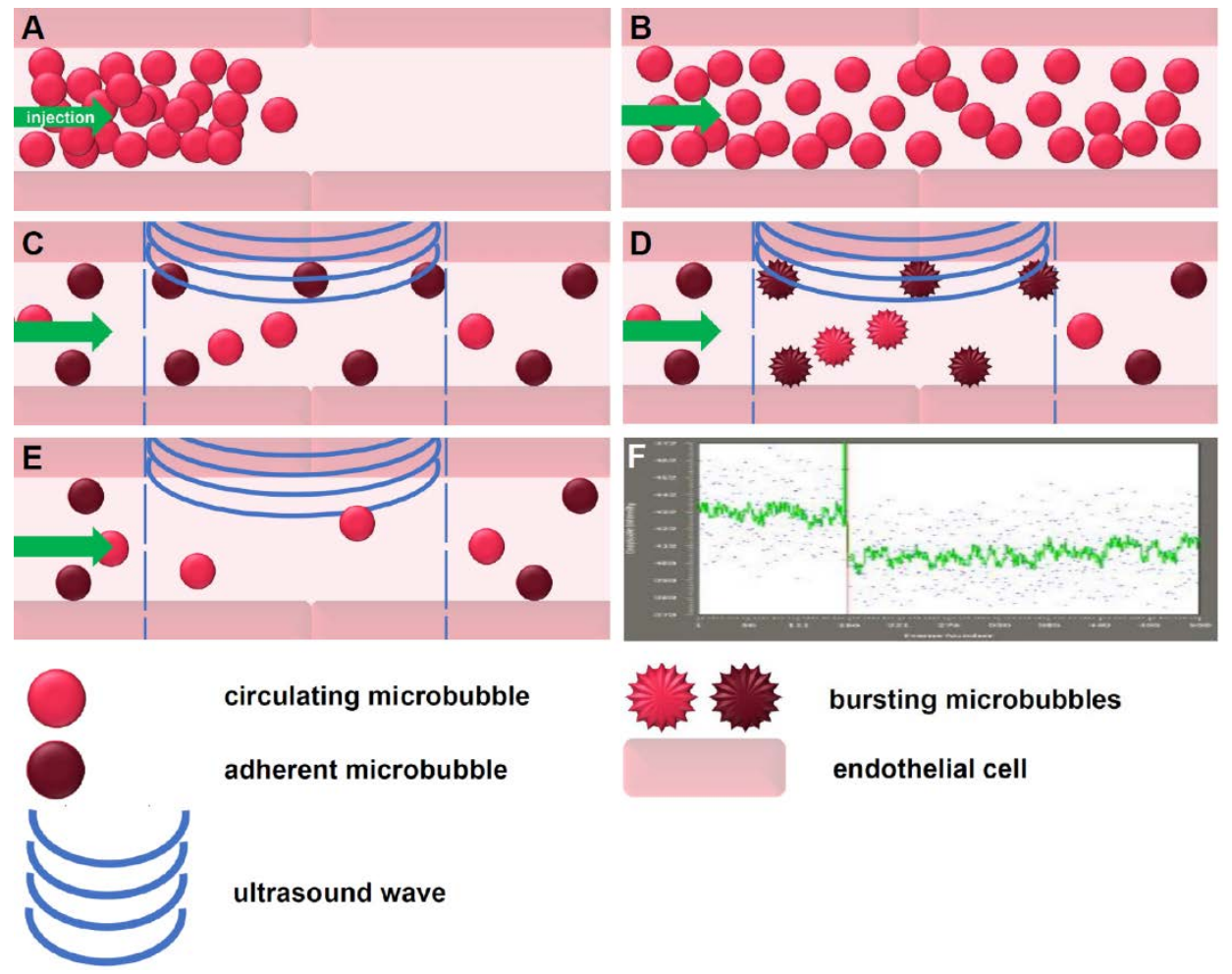

Figure 5: Schematic representation of molecular US imaging. (A) MB suspension injection into the bloodstream followed by blood pool distribution of MBs (B). (C) MB attachment to their endothelial target, and the destruction pulse (D) fallowed by imaging of the replenishing process (E). (F) A destruction curve extracted from the US device after processing the data acquisition.

\section{Two-photon laser scanning microscopy}

\section{Principles}

Fluorescence microscopy refers to any type of microscopy that uses fluorescence tostudy properties of organic or inorganic substances to generate an image. Fluorescence is the process of photon emission by a molecule that has absorbed a photon.

The fluorescence microscope is a heavily used, broad spectrum imaging tool with extraordinary spatial and temporal resolution. It is used to investigate dynamic processes in tissues, cells, and even subcellular compartments. Using distinct 
fluorescent markers (probes), labeling specific subcellular structures, various cellular compartments can be imaged sequentially or simultaneously. The term "fluorescence microscope" refers to a wide spectrum of microscopes, from a simple set- up like an epifluorescence microscope, or a more complicated one as a confocal microscope, to a multi photon microscope.

Epifluorescence microscopes, routinely used in immunohistology of thin slices of fixed tissue, present a two-dimensional image without providing any z-dimensional information. The excitation light pentrates the entire depth of the tissue causing not only fluorescence emission in the focal plane, but also above and under. On the contrary, confocal microscopes limit the amount of out-of-focus fluorescence that reaches the detector by varying the radius of the pinhole, thereby promoting imaging on the z-axis, rendering image stacks at different depths of tissues or cells at higher resolution. However, the pinhole reduces the amount of emitted fluorophores and can result in weak fluorescence signals. A major limitation of confocal microscopy is imaging speed. Using point-to-point laser scanner, image acquisition is significantly slower than epifluorescence microscopes. Imaging speed increases proportionally with the amount of fluorophores, as each fluorophore is excited seperatly to avoid bleach-over of different excition laser into the detectors. This so-called sequential imaging slows down confocal microscopy and limits its capacity for live cell imaging. Conventional linear single-photon confocal microscopy comes to its limits when imaging thicker samples because of the UV/visible wavelengths $(350-650 \mathrm{~nm})$ which cause scattering in biological tissues. As a consequence, deeper in tissue unwanted out-of-focus light would pass the pinholes and consequently decrease resolution in all directions. Therefore, confocal fluorescence imaging is restricted to an imaging depth of around $50 \mu \mathrm{m}$, the exact value depending on tissue.

In comparison to linear single-photon confocal microscopy, in two-photon microscopy, a fluorophore achieves the transition from its ground to an excited state by the near-simultaneous $\left(\sim 10^{-18} \mathrm{~s}\right)$ absorption of two (near) infrared (NIR) $(\sim 800$ $1000 \mathrm{~nm}$ ) photons. These two photons have approximately half the energy, and thus double the wavelength, of the photon required for single photon excitation. The longer excitation wavelengths dramatically increases tissue penetration depth and 
allows for long-term imaging without compromised tissue viability. Most importantly, however, in two-photon microscopy, the excitation (and thus fluorescence) is restricted to the focal spot, since only in this spot the excitation probability is high enough to achieve fluorescence. This localized excitation reduces out-of-focus fluorescence. As a consequence, even deeper in tissue resolution is maintained,as there is no out-of-focus fluorescence. Also, this increases penetration depth and limits photo-toxicity and photo-bleaching to the focal plane. Therefore, multiphoton imaging is the perfect tool for deep tissue imaging in thick tissue specimens, such as brain slices or tumor or lymph node explants, as well as intravital research of small animal models.

An additional advantage of multiphoton excitation is that probes with different excitation wavelengths under single photon excitation, can have overlapping twophoton excitation wavelengths. This is caused by the in general broadened twophoton excitation spectra, allowing multi-dye imaging at a single two-photon excitation wavelength. Multi-dye imaging increases the number of channels possible using a single excitation wavelength without the use of filter wheels and can be advantageous for interaction studies between cells or subcellular components. It furthermore increases the speed at which images from various probes can be registered. Finally, non-linear excitation has the advantage of allowing visualization of conventional dyes for tissue staining, as well the visualization of tissue by exploiting second and third harmonic generation (THG). In second harmonic generation (SHG), 2 photons interact simultaneously with a non-centrosymmetric target (such as collagen, myosin, and tubulin), leading to the emission of a single photon with twice the energy. The SHG is a scattering process, providing photon emission without absorption and thus without photo-bleaching. The THG is a nonlinear process as well, which requires the interaction of 3 photons for the emission of a single photon with one third of the excitation wavelength. THG is created at sites of refractive index changes. However, current standard multiphoton microscopes have a limited excitation wavelength spectrum and thus do not allow THG imaging.

Two-photon laser scanning microscopy (TPLSM) is widely used in the biomedical field. Its advantages have established TPLSM as a valuable tool for deep tissue 
imaging ex vivo, in vivo, and in long-term measurements. Moreover, imaging vital tissue has one distinct advantage, it reduces the probability of artifacts that accompany the various steps of tissue preparation for regular histology and immunohistochemistry, e.g. fixation, cutting, and staining.

\section{Application in cardiovascular imaging}

TPLSM has been successfully used on a wide range of viable tissues ex vivo, and as well motionless tissues in vivo, such as brain ${ }^{41,42}$, kidney ${ }^{43,44}$, and tumors ${ }^{45}$. The development of triggering systems and fast imaging methods determined the success of intravital imaging of the fast pulsating heart and major arteries in animal models. Therefore, TPLSM has also proved its value is cardiovascular research, being used not only for imaging small vessels such as vasa vasorum associated to the atherosclerotic plaque ${ }^{46}$ but as well imaging strong pulsing major blood vessels ${ }^{47}$. Many of the limitations of conventional single-photon imaging, such as image blurriness due to out-of-focus fluorescence, penetration depth of less than 50 $\mu \mathrm{m}$, photo-toxicity, photo-activation, and photobleaching have been overcome by the introduction of TPLSM for imaging thick and scattering tissues such as heart and big vessels. Moreover, it allows the simultaneous acquisition of functional, structural, and molecular information from intact blood vessels, and beating hearts. Due to its advantage of visualizing multiple fluorophores and deep tissue penetration, the TPLSM has been successfully employed for the visualization of fluorescent labeled leukocyte sub-populations trafficking through the endothelium ${ }^{48}$, and thus has found its applicability in imaging biological processes involved in the initiation and progression of atherosclerosis ${ }^{49,50}$. Additionally, TPLSM been employed in tracking the functional fate of a variety of donor cells following their intracardiac transplantation ${ }^{51}$. Moreover, it has been used for providing structural and quantitative information regarding healthy, infarcted, and regenerating hearts by employing the $\mathrm{SHG}^{52}$.

In conclusion, intravital cardiovascular TPLSM imaging has the potential to further bridge in vitro or in situ with preclinical imaging modalities. 


\section{Research question, aim and outline of the thesis}

Atherosclerosis has major health implications, e.g stroke and myocardial infarction. Conventional treatment options of atherosclerotic plaques consist of balloon angioplasty with/without stent placement, or endarterectomy, followed by long-term dual antiplatelet therapy. However, all current interventions injure the endothelial barrier, promoting neointima formation. As a result, patients are commonly monitored employing functional and morphological US, to illustrate the anatomy and quantify the degree of vessel re-obstruction after revascularization procedures. Therefore, routinely used monitoring protocols following revascularization procedures can only provide information on the extent of arterial obstruction, but not on the biological state of the endothelial barrier. Consequently, imaging protocols allowing the assessment of endothelial dysfunction or recovery would be of great interest and high clinical impact. For this purpose, distinct adhesion molecules involved in endothelial recovery process could be utilized for molecular imaging purposes.

Molecular imaging of activated endothelial cells in major vessels, such as carotid arteries is a daunting task due to the high flow and shear rates. Therefore, US molecular imaging studies on atherosclerosis have tried to overcome these impediments by various methods, including the use of multiple targeting ligands ${ }^{53}$. However, in vivo binding assessment of targeted MB has been so far limited to the aortic $\operatorname{root}^{54,55}$ or $\operatorname{arch}^{56}$, which characteristically have-larger diameters (up to 3 fold) and significantly lower shear stress than carotid arteries ${ }^{57}$.

Thus, the overall aim of this thesis was to show that molecular US employing targeted MB can be applied for assessing endothelial dysfunction of murine carotid arteries at physiological and pathological flow and shear rates. Initially, in the first study in Chapter 2 we evaluated the in vitro and ex vivo binding capacity of ICAM-1 targeted MB to TNF- $\alpha$ stimulated endothelial cells expressing ICAM-1 at physiological and pathological flow and shear rates, followed by their detection using molecular US and TPLSM. Binding capacity of ICAM-1-targeted MB to carotid endothelium was further validated in vivo by employing molecular US imaging. 
Based on these data, we transferred to an animal model that reflected a relevant clinical situation found in human patients: endothelial injury after revascularization procedures. Therefore, the procedure describing the murine model of arterial denudation, which resembles the clinical situation found in human patients is presented in detail in Chapter 3. Subsequently, in Chapter 4 we report on the monitoring of vascular recovery after endothelial denudation by employing VCAM-1 targeted MB (MBVCAM-1) for molecular US imaging. To validate the specificity of MBVCAM-1 binding to the luminal side of the injured carotid artery, TPLSM imaging was employed. Finally, we show the applicability of molecular US imaging in a murine model of native carotid atherosclerotic plaque by using JAM-A as marker for acute change in blood flow with consequences on endothelial activation, in Chapter 5. 


\section{References}

1. Reitsma S, Slaaf DW, Vink H, van Zandvoort MA, oude Egbrink MG. The endothelial glycocalyx: composition, functions, and visualization. Pflugers Arch. 2007;454(3):345-359.

2. Paoletti R, Gotto AM, Jr., Hajjar DP. Inflammation in atherosclerosis and implications for therapy. Circulation. 2004;109(23 Suppl 1):III20-26.

3. Hunt BJ, Jurd KM. Endothelial cell activation. A central pathophysiological process. BMJ. 1998;316(7141):1328-1329.

4. Blankenberg S, Barbaux S, Tiret L. Adhesion molecules and atherosclerosis. Atherosclerosis. 2003;170(2):191-203.

5. Cybulsky MI, Gimbrone MA, Jr. Endothelial expression of a mononuclear leukocyte adhesion molecule during atherogenesis. Science. 1991;251(4995):788-791.

6. Li H, Cybulsky MI, Gimbrone MA, Jr., Libby P. An atherogenic diet rapidly induces VCAM-1, a cytokine-regulatable mononuclear leukocyte adhesion molecule, in rabbit aortic endothelium. Arterioscler Thromb. 1993;13(2):197204.

7. Packard RR, Libby P. Inflammation in atherosclerosis: from vascular biology to biomarker discovery and risk prediction. Clin Chem. 2008;54(1):24-38.

8. Galkina E, Ley K. Vascular adhesion molecules in atherosclerosis. Arterioscler Thromb Vasc Biol. 2007;27(11):2292-2301.

9. Ley K, Miller YI, Hedrick CC. Monocyte and macrophage dynamics during atherogenesis. Arterioscler Thromb Vasc Biol. 2011;31(7):1506-1516.

10. Liberale L, Dallegri F, Montecucco F, Carbone F. Pathophysiological relevance of macrophage subsets in atherogenesis. Thrombosis and haemostasis. 2017;117(1):7-18.

11. Libby P, Ridker PM, Maseri A. Inflammation and atherosclerosis. Circulation. 2002;105(9):1135-1143.

12. Spagnoli LG, Bonanno E, Sangiorgi G, Mauriello A. Role of inflammation in atherosclerosis. J Nucl Med. 2007;48(11):1800-1815.

13. Baleanu-Curaj A. A new therapeutic strategy for inhibiting arterial restenosis and myocardial ischemia/reperfusion injury in mice. Doctoral thesis 
(Dr.med), RWTH Aachen University, The Institute for Experimental Molecular Imaging. 2014.

14. Miller YI, Chang MK, Binder CJ, Shaw PX, Witztum JL. Oxidized low density lipoprotein and innate immune receptors. Curr Opin Lipidol. 2003;14(5):437445.

15. Lee RT, Yamamoto $C$, Feng $Y$, et al. Mechanical strain induces specific changes in the synthesis and organization of proteoglycans by vascular smooth muscle cells. J Biol Chem. 2001;276(17):13847-13851.

16. Oh J, Riek AE, Weng $S$, et al. Endoplasmic reticulum stress controls $M 2$ macrophage differentiation and foam cell formation. $J$ Biol Chem. 2012;287(15):11629-11641.

17. Asakura T, Karino T. Flow patterns and spatial distribution of atherosclerotic lesions in human coronary arteries. Circ Res. 1990;66(4):1045-1066.

18. Malek AM, Alper SL, Izumo S. Hemodynamic shear stress and its role in atherosclerosis. JAMA. 1999;282(21):2035-2042.

19. Feldman $\mathrm{CL}$, Stone $\mathrm{PH}$. Intravascular hemodynamic factors responsible for progression of coronary atherosclerosis and development of vulnerable plaque. Curr Opin Cardiol. 2000;15(6):430-440.

20. Abou-Elkacem L, Bachawal SV, Willmann JK. Ultrasound molecular imaging: Moving toward clinical translation. Eur J Radiol. 2015;84(9):1685-1693.

21. Postema M, Gilja OH. Contrast-enhanced and targeted ultrasound. World J Gastroenterol. 2011;17(1):28-41.

22. Hangiandreou NJ. AAPM/RSNA physics tutorial for residents. Topics in US: B-mode US: basic concepts and new technology. Radiographics. 2003;23(4):1019-1033.

23. Chan V, Perlas A. Basics of Ultrasound Imaging. in Atlas of UltrasoundGuided Procedures in Interventional Pain Management (ed. Narouze, S.N.E.) 13-19 ( Springer Science+Business Media, 2011).

24. Mace E, Montaldo G, Osmanski BF, Cohen I, Fink M, Tanter M. Functional ultrasound imaging of the brain: theory and basic principles. IEEE Trans Ultrason Ferroelectr Freq Control. 2013;60(3):492-506.

25. Hahn R. Recent advances in echocardiography for valvular heart disease. F1000Res. 2015;4(F1000 Faculty Rev):914. 
26. Cho JY, Kim KH. Evaluation of Arterial Stiffness by Echocardiography: Methodological Aspects. Chonnam Med J. 2016;52(2):101-106.

27. Markley RR, Ali A, Potfay J, Paulsen W, Jovin IS. Echocardiographic Evaluation of the Right Heart. J Cardiovasc Ultrasound. 2016;24(3):183-190.

28. Deshpande N, Needles A, Willmann JK. Molecular ultrasound imaging: current status and future directions. Clin Radiol. 2010;65(7):567-581.

29. Curaj A, Wu Z, Fokong S, et al. Noninvasive molecular ultrasound monitoring of vessel healing after intravascular surgical procedures in a preclinical setup. Arteriosclerosis, thrombosis, and vascular biology. 2015;35(6):13661373.

30. de Jong N, Bouakaz A, Frinking P. Basic acoustic properties of microbubbles. Echocardiography. 2002;19(3):229-240.

31. Stride E. Physical principles of microbubbles for ultrasound imaging and therapy. Cerebrovasc Dis. 2009;27 Suppl 2:1-13.

32. Alkan-Onyuksel H, Demos SM, Lanza GM, et al. Development of inherently echogenic liposomes as an ultrasonic contrast agent. J Pharm Sci. 1996;85(5):486-490.

33. Marsh JN, Partlow KC, Abendschein DR, Scott MJ, Lanza GM, Wickline SA. Molecular imaging with targeted perfluorocarbon nanoparticles: quantification of the concentration dependence of contrast enhancement for binding to sparse cellular epitopes. Ultrasound in medicine \& biology. 2007;33(6):950-958.

34. Korosoglou G, Behrens S, Bekeredjian R, et al. The potential of a new stable ultrasound contrast agent for site-specific targeting. An in vitro experiment. Ultrasound in medicine \& biology. 2006;32(10):1473-1478.

35. Gessner R, Dayton PA. Advances in molecular imaging with ultrasound. Mol Imaging. 2010;9(3):117-127.

36. Sirsi S, Borden M. Microbubble Compositions, Properties and Biomedical Applications. Bubble Sci Eng Technol. 2009;1(1-2):3-17.

37. Kiessling F, Fokong S, Bzyl J, Lederle W, Palmowski M, Lammers T. Recent advances in molecular, multimodal and theranostic ultrasound imaging. Adv Drug Deliv Rev. 2014;72:15-27. 
38. Goertz DE, Needles A, Burns PN, Foster FS. High-frequency, nonlinear flow imaging of microbubble contrast agents. IEEE Trans Ultrason Ferroelectr Freq Control. 2005;52(3):495-502.

39. de Jong N, Frinking PJ, Bouakaz A, Ten Cate FJ. Detection procedures of ultrasound contrast agents. Ultrasonics. 2000;38(1-8):87-92.

40. Forsberg F, Shi WT, Goldberg BB. Subharmonic imaging of contrast agents. Ultrasonics. 2000;38(1-8):93-98.

41. Helmchen $\mathrm{F}$, Waters $\mathrm{J}$. Ca2+ imaging in the mammalian brain in vivo. Eur $\mathrm{J}$ Pharmacol. 2002;447(2-3):119-129.

42. Levene MJ, Dombeck DA, Kasischke KA, Molloy RP, Webb WW. In vivo multiphoton microscopy of deep brain tissue. $J$ Neurophysiol. 2004;91(4):1908-1912.

43. Molitoris BA, Sandoval RM. Multiphoton imaging techniques in acute kidney injury. Contrib Nephrol. 2010;165:46-53.

44. Molitoris BA, Sandoval RM. Intravital multiphoton microscopy of dynamic renal processes. Am J Physiol Renal Physiol. 2005;288(6):F1084-1089.

45. Tozer GM, Ameer-Beg SM, Baker J, et al. Intravital imaging of tumour vascular networks using multi-photon fluorescence microscopy. Adv Drug Deliv Rev. 2005;57(1):135-152.

46. Rademakers T, Douma K, Hackeng TM, et al. Plaque-associated vasa vasorum in aged apolipoprotein E-deficient mice exhibit proatherogenic functional features in vivo. Arterioscler Thromb Vasc Biol. 2013;33(2):249256.

47. Megens RT, Reitsma S, Prinzen L, et al. In vivo high-resolution structural imaging of large arteries in small rodents using two-photon laser scanning microscopy. J Biomed Opt. 2010;15(1):011108.

48. Pietronigro E, Zenaro E, Constantin G. Imaging of Leukocyte Trafficking in Alzheimer's Disease. Front Immunol. 2016;7:33.

49. Schmitt MM, Megens RT, Zernecke A, et al. Endothelial junctional adhesion molecule-a guides monocytes into flow-dependent predilection sites of atherosclerosis. Circulation. 2014;129(1):66-76.

50. Wu Z, Curaj A, Fokong S, et al. Rhodamine-loaded intercellular adhesion molecule-1-targeted microbubbles for dual-modality imaging under 
controlled shear stresses. Circulation. Cardiovascular imaging. 2013;6(6):974-981.

51. Scherschel JA, Rubart M. Cardiovascular imaging using two-photon microscopy. Microsc Microanal. 2008;14(6):492-506.

52. Wallenburg MA, Wu J, Li RK, Vitkin IA. Two-photon microscopy of healthy, infarcted and stem-cell treated regenerating heart. $\mathrm{J}$ Biophotonics. 2011;4(5):297-304.

53. Ferrante EA, Pickard JE, Rychak J, Klibanov A, Ley K. Dual targeting improves microbubble contrast agent adhesion to VCAM-1 and P-selectin under flow. J Control Release. 2009;140(2):100-107.

54. Nahrendorf M, Jaffer FA, Kelly KA, et al. Noninvasive vascular cell adhesion molecule-1 imaging identifies inflammatory activation of cells in atherosclerosis. Circulation. 2006;114(14):1504-1511.

55. Nahrendorf M, Keliher E, Panizzi $P$, et al. 18F-4V for PET-CT imaging of VCAM-1 expression in atherosclerosis. JACC Cardiovasc Imaging. 2009;2(10):1213-1222.

56. Kaufmann BA, Sanders JM, Davis C, et al. Molecular imaging of inflammation in atherosclerosis with targeted ultrasound detection of vascular cell adhesion molecule-1. Circulation. 2007;116(3):276-284.

57. Trachet B, Swillens A, Van Loo D, et al. The influence of aortic dimensions on calculated wall shear stress in the mouse aortic arch. Comput Methods Biomech Biomed Engin. 2009;12(5):491-499. 
General introduction 



\section{Chapter 2}

\section{Rhodamine-loaded ICAM-targeted}

\section{microbubbles for dual-modality imaging under controlled shear}

\section{stresses}

Zhuojun Wu*, Adelina Curaj", Stanley Fokong, Elisa A. Liehn, Christian Weber, Twan Lammers, Fabian Kiessling, Marc van Zandvoort ("equal contribution)

Circ Cardiovasc Imaging. 2013 Nov;6(6):974-81.

doi: 10.1161/CIRCIMAGING.113.000805.

PMID: 24036383 


\section{Abstract}

Background: The ability to image incipient atherosclerosis is based on the early events taking place at the endothelial level. We hypothesized that the expression of intercellular adhesion molecule-1 (ICAM-1) even in vessels with very high flow rates can be imaged at the molecular level using two complementary imaging techniques: Two-Photon Laser Scanning Microscopy (TPLSM) and Contrast-Enhanced Ultrasound (CEUS).

Methods and Results: Using TPLSM and CEUS, ICAM-1-targeted and rhodamine-loaded microbubbles (MBICAM-1) were shown to be specifically bound to TNFa-stimulated human umbilical vein endothelial cells (HUVEC) and murine carotid arteries (44 wild type mice) at shear stresses ranging from $1.25 \mathrm{dyn} / \mathrm{cm} 2$ to $120 \mathrm{dyn} /$. MBICAM-1 $_{1}$ bound 8 times more efficient $(p=0.016)$ to stimulated HUVECs than to unstimulated cells and 14 times more than non-targeted MB ( $p$ $=0.016)$. In excised carotids, binding efficiency did not significantly decrease when increasing the flow rate from $0.25 \mathrm{ml} / \mathrm{min}$ to $0.6 \mathrm{ml} / \mathrm{min}$. Higher flow rates $(0.8 \mathrm{ml} / \mathrm{min}$ and $1 \mathrm{ml} / \mathrm{min}$ ) showed significantly reduced $\mathrm{MB}$ retention, by $38 \%$ ( $\mathrm{p}$ $=0.03)$ and $55 \%(p=0.03)$, respectively. Ex vivo results were translatable in vivo, confirming that $\mathrm{MB}_{\triangle \mathrm{ICAM}-1}$ are able to bind specifically to the inflamed carotid artery endothelia under physiological flow conditions and to be noninvasively detected using CEUS.

Conclusions: Our data provide groundwork for the implementation of molecular ultrasound imaging in vessels with high shear stress and flow rates, as well as for the future development of image-guided therapeutic interventions, and multiphoton microscopy as the appropriate method of validation. 


\section{Introduction}

Atherosclerosis, a chronic inflammatory disorder developing in arterial vessels ${ }^{1}$, is mainly caused by alterations in arterial shear stress ${ }^{2}$, followed by subendothelial accumulation of lipids and inflammatory cells leading to wall thickening and segmental lumen narrowing. The detection of atherosclerosis using imaging modalities such as CT, MRI and US is challenging, and in many cases only possible in the late stages of disease progression, when the plaque narrows the arterial lumen and determines clinical manifestations ${ }^{3}$. The main challenge is to diagnose and ultimately to intervene therapeutically in the atherosclerotic process during the early stages of disease progression.

The endothelium does not only play a prominent role in the early stage of atherosclerosis, but also after conventional balloon angioplasty. Abnormal shear stress and the aggressive fat accumulation in the vessel wall are followed by strong endothelial activation. Activated endothelial cells upregulate surface adhesion molecules, such as vascular cell adhesion molecule-1 (VCAM-1) and intercellular adhesion molecule-1 (ICAM-1) that can initiate leukocyte recruitment and transmigration, thereby contributing to the plaque growth ${ }^{4-6}$. These distinct adhesion molecules can be utilized for molecular imaging purposes. In this context, due to the fact that these are intravascular markers, ultrasound molecular imaging with target-specific $\mu \mathrm{m}$-sized gas bubbles (which stay strictly intravascular) present a suitable method for the imaging of endothelial dysfunction. However, molecular imaging of activated endothelium in major vessels, such as carotid arteries is a daunting task due to the high flow and shear rates. Therefore, ultrasound molecular imaging studies on atherosclerosis have tried to overcome these obstacles by various methods, including: modulating the microbubbles (MB) surface coverage with ligands ${ }^{7}$, using multiple targeting ligands ${ }^{8}$ etc., mostly in vitro on cell cultures. So far, in vivo binding assessment of targeted MB has been limited to the aortic $\operatorname{arch}^{9}$, which characteristically has a larger diameter (up to 3 fold) and significantly lower shear stress than carotid arteries $^{10}$.

The aim of this investigation is to show that Contrast-Enhanced Ultrasound 
(CEUS) using targeted MB can be applied for molecular imaging of carotid incipient atherosclerosis at physiological flow and shear rates. Polymeric MB have been shown to be suitable contrast agents for functional and molecular ultrasound imaging ${ }^{11}$. The target of choice was ICAM-1 because of its physiological relevance in the early stages of plaque development ${ }^{12}$ but also because of its Tumor Necrosis Factor alpha (TNFa) dependent upregulation ${ }^{13}$. Using ICAM-1-targeted and rhodamine-labeled $\mathrm{MB}^{14}$, the binding of $\mathrm{MB}$ to inflamed endothelial surfaces was systematically investigated under different flow and shear-stress conditions, visualized by CEUS and quantitatively validated by Two-Photon Laser Scanning Microscopy (TPLSM). These results serve as a proof of concept that CEUS with targeted MB can be performed in carotid arteries and stress the appropriateness of TPLSM as validation method in tissues.

\section{Materials and Methods}

\section{Synthesis of rhodamine-loaded target-specific microbubbles}

Rhodamine encapsulated poly (n-butyl cyanoacrylate) (PBCA) MB were synthesized as previously described ${ }^{15}$. In brief, $3 \mathrm{~mL}$ of monomeric butyl-2cyanoacrylate (BCA; Special polymer Itd.) were added drop wise to $300 \mathrm{~mL}$ of an aqueous solution of $1 \%$ Triton $\mathrm{X}-100$ (Sigma-Aldrich) at $\mathrm{pH} 2.5$. Subsequently, rhodamine- $\mathrm{B}$, was dissolved in $300 \mu \mathrm{L}$ of distilled water and added to the mixture. Using an Ultra Turrax, the mixture was then stirred for 60 minutes at $10000 \mathrm{rpm}$. The resulting rhodamine-loaded MB suspension was washed several times by flotation to remove the excess rhodamine. For the conjugation of Streptavidin (Applichem) to the MB surface, the ester groups were partially hydrolyzed. Subsequently, streptavidin was conjugated to the MB by a 1-ethyl-3-(3dimethylaminopropyl)carbidiimide hydrochloride (Sigma Aldrich) reaction between the amine group of the streptavidin and the carboxylic group of the MB surface, followed by 3 flotation steps. Size distribution and MB concentration were determined with the particle counter Multisizer 3 (Beckman-Coulter) (Supplementary Figure S2). 


\section{Antibody labeling}

$1 \times 10^{7}$ streptavidin-coated MB in HEPES/Triton buffer $(\mathrm{pH} \mathrm{7.0)}$ were mixed with $5 \mu \mathrm{g}$ of biotinylated ICAM-1 antibodies and incubated at room temperature for 30 minutes. The conjugated MB were separated from excess antibodies by centrifugation (500 rpm for $2 \mathrm{~min}$ ) and re-suspended in $100 \mu \mathrm{L}$ HEPES/Triton buffer.

\section{Cell culture}

Human umbilical vein endothelial cells (HUVEC) were grown and maintained in endothelial cell growth medium (ECGM II), supplemented with $10 \%(\mathrm{v} / \mathrm{v})$ heat inactivated fetal bovine serum, endothelial cell growth supplement and $1 \%(\mathrm{v} / \mathrm{v})$ gentamicin at $37^{\circ} \mathrm{C}$ in a $5 \% \mathrm{CO}_{2}$ humidified atmosphere. All reagents were purchased from Promocell.

\section{In vitro flow chamber}

$100 \mu \mathrm{l}$ of HUVEC were seeded $\left(1 \times 10^{6}\right.$ cells $\left./ \mathrm{mL}\right)$ on collagen-coated $35-\mathrm{mm}$ Petri dishes. The cells were incubated with $40 \mathrm{ng}$ of recombinant human TNFa (PeproTech) for 4 hours prior to MB perfusion. The cells were incubated with WGA-AF488 (1:1000) and SYTO 41 (1:1000) (Invitrogen) for 45 minutes and then washed with 1x PBS. The Petri 35-mm dishes were mounted into a customized parallel wall flow chamber in a custom silicon tube closed perfusion system (standard silicon tubing $0.76 \mathrm{~mm}$ inner diameter - Helixmark). Using a peristaltic pump (Gilson Inc), a continuous flow rate of $0.25 \mathrm{ml} / \mathrm{min}$ was set. $1 \times 10^{7}$ ICAMconjugated MB were injected into the perfusion system and a closed loop circulation was performed for 10 minutes, followed by a 10-minute washing step using 1x PBS with an open loop. Fluorescence images were obtained subsequently using a Axio Imager M2 fluorescence microscope (Carl Zeiss AG, Germany). 


\section{Immunohistology}

For immunohistology, perfusion fixation with 4\% Paraformaldehyde (P-6148, Sigma) and paraffin embedding, either PBS perfusion followed by Tissue-Tek ${ }^{\circledR}$ O.C.T. ${ }^{\mathrm{TM}}$ (4583, Sakura) embedding and snap-freezing was performed. Serial tissue sections $(5 \mu \mathrm{m})$ starting with the bifurcation were obtained from isolated carotids and stained for ICAM-1 (Santa Cruz Biotechnology Inc: sc-52553), VCAM-1 (16-1061, eBioscience), P-selectin (sc-6941), E-selectin (sc-6939) and PECAM-1/CD31 (sc-1506) expression. Biotinylated secondary antibodies (antirat IgG BA-4000 and anti-goat IgG BA-5000, Vector Laboratoies) followed by Fluorescein Streptavidin (SA-5001, Vector Laboratories) conjugation were used for specific staining visualization and sections were analyzed using Diskus software (Hilgers).

\section{Murine model of endothelial activation}

All animal experiments were approved by local authorities and complied with German animal protection law. Endothelial activation was induced in eight-weekold male CD-1 wild type mice via intraperitoneal (i.p.) injection of recombinant murine TNFa (PeproTech) $0.067 \mathrm{mg} / \mathrm{kg}$. Control mice and TNFa-stimulated mice were sacrificed by i.p. injection of anesthetic overdose (400 mg/kg ketamine, 40 $\mathrm{mg} / \mathrm{kg}$ xylazine) 4 hours after TNFa injection and both (left and right) common carotids excised by a cervico-thoracal incision. Animals were separated into 4 groups (immunohistochemistry ( $n=6)$, ex-vivo TPLSM ( $n=20)$, ex vivo CEUS ( $n=$ 10) and in vivo CEUS $(n=10))$, each group was separated into 2 subgroups (control, TNFa-stimulated). The total number of animal $=44$.

\section{Ex vivo perfusion}

Mice were sacrificed by i.p. overdose injection of anesthetic. The common carotid arteries $(6-7 \mathrm{~mm})$ were excised and freed from adipose and connective tissue, without damaging the blood vessel. Handling of the blood vessel was restricted only to both ends. In order to avoid air bubbles within the lumen, the blood vessels were preserved at $4^{\circ} \mathrm{C}$ in $1 \mathrm{x}$ Hanks Balanced Salt Solution (HBSS, pH 7.4, Life 
technology) before the mounting procedure. Each excited carotid was mounted in a customized flow chamber, filled with HBSS $\left(37^{\circ} \mathrm{C}\right)^{16}$. The carotid was mounted on each side onto glass pipettes (tip diameter 120-150 $\mu \mathrm{m}$ ). Luminal blood residues were removed by flushing with 1x HBSS. In order to correct for shortening and deflation of the carotid, the blood vessel was pressurized (60 $\mathrm{mmHG}$ ) and stretched. Fresh blood collected from healthy volunteers was anticoagulated with heparin $(10 \mathrm{U} / \mathrm{ml})$ - EDTA (1.2 - $2 \mathrm{mg}$ EDTA $/ \mathrm{ml})$ and infused through the carotids at a flow rate of $0.25 \mathrm{ml} / \mathrm{min}$ for 2 minutes.

All flow experiments were conducted at $37^{\circ} \mathrm{C}$ using flow rates ranging from 0.25 $\mathrm{ml} / \mathrm{min}$ to $1 \mathrm{ml} / \mathrm{min}(0.25,0.6,0.8$ and $1 \mathrm{ml} / \mathrm{min}$ in a custom silicon tube perfusion system using (Standard Silicon Tubing $0.76 \mathrm{~mm}$ inner diameter - Helixmark) and flow was established using a peristaltic pump in the same direction as under physiological conditions. Either targeted or control MB $\left(10^{7} \mathrm{ml}^{-1}\right)$, suspended in whole blood, were infused for 10 minutes, followed by 10 minutes washing using human blood (ex vivo ultrasound invesigations were performed without removing the circulating MB). For competitive binding studies, the TNF $\alpha$ stimulated carotid was perfused with 20 fold higher concentration of free ICAM antibody $(100 \mu \mathrm{g}, 2$ $\mu \mathrm{g} / \mu \mathrm{l})$ for 10 minutes before MB injection. The carotid was then imaged using CEUS or TPLSM. The number of animal used $n=20$.

\section{Ex vivo ultrasound imaging}

Tissue preparation was performed as described. High-resolution imaging of the carotid arteries was performed with a high-frequency $(21 \mathrm{MHz})$ imaging system (Vevo 2100, VisualSonics Inc) using a long-axis imaging plane. Imaging was performed as described in Kiessling et al. (2012) ${ }^{17}$. We considered that after 10 minutes of continuous flow, MB distribution is homogeneous in the circulating blood and imaging can be performed. The cumulative signal of circulating and bound $\mathrm{MB}$ can be detected in the imaging frames before the destructive pulse. MB specific signal was distinguished by Nonlinear Contrast Mode data acquisition. An imaging sequence of 100 seconds (10 frames/second) was acquired with low-power imaging (4\%), followed by a short increase of the acoustic amplitude to $100 \%$ power at the end of the first third of the imaging 
sequence in order to destroy the MB in the field of view. The early replenishment of signal intensity represents the renewed inflow and binding of MB. MB-derived contrast enhancement does not reach pre-destruction levels due to reduced availability of binding sites. The destruction of bound MB does not release the receptor but keeps it occupied with the antibody bound to the MB-residue. Image analysis was performed by subtracting the mean contrast power for predestructive frames (bound + circulating $\mathrm{MB}$ ) from the post-destructive frames (only circulating MB). The mean contrast decrease represents the amount of signal generated by retained MB. The number of animals used for this studies is $n=8$.

\section{In vivo ultrasound imaging}

Mice were stimulated with TNFa, according to our murine model of endothelial activation. In vivo molecular ultrasound measurements were performed using a small animal high frequency ultrasound system (Vevo 770) and a $45 \mathrm{Mhz}$ transducer. Seven minutes after injection of $100 \mu \mathrm{l}$ of ICAM-1-targeted MB (MBICAM-1) $\left(2 \times 10^{7}\right)$ into the tail vein, ultrasound imaging in Contrast mode was performed analogously to the ex vivo flow chamber experiments. The number of animal used for this study is $n=10$.

\section{Two-photon laser scanning microscopy}

The carotid artery within the flow chamber was imaged using an Olympus FV1000MPE multiphoton microscopy system (Mai Tai DeepSee pulsed Ti:Sapphire laser with $140 \mathrm{fs}$ pulsewidth at an excitation wavelength of $800 \mathrm{~nm}$ and a $25 \mathrm{x}$ water dipping objective (NA $=1.05$, WD $=2 \mathrm{~mm}$ )). Three internal photon multiplier tubes were used to detect the fluorescence signals, and filters were adjusted to the corresponding spectra: $390-460$ for collagen second harmonic imaging, 490-540 for elastin autofluorescence and 590-620 for rhodamine. TPLSM images were analyzed using Image-Pro Analyzer 7.0 software (Media Cybernetis, Inc.) and Imaris software (Bitplane). 


\section{Vessel diameter estimation and shear stress calculation}

Vessel diameter estimation was performed as described in Van Triest et al. $(2010)^{18}$. Accordingly, shear stress for each carotid was calculated based on the Haagen-Poisseuille equation. Blood viscosity at $37^{\circ} \mathrm{C}$ was estimated at 0.004 Pa*s.

\section{Statistical Analysis}

MB adhesion efficiency both in vitro, ex vivo and in vivo $(n=5)$ were analyzed using the Mann-Whitney test, $p<0.05$ is considered significant. Error bars shown on graphs are the standard error of the mean. To correct for multiple group comparison, we performed the Bonferroni correction. Statistical analysis was performed using GraphPad Prism 5.0 (GraphPad Software)

\section{Results}

\section{Analysis of biomarker expression after carotid artery stimulation}

In order to characterize the molecular expression of endothelial surface receptors after TNFa stimulation and to determine the most suitable targeting ligand for the $\mathrm{MB}$ binding studies, immunostainings on TNFa-stimulated carotids from wild type mice were performed, showing ICAM-1, P-selectin, E-selectin and PECAM$1 / C D 31$ expression on the luminal surface of activated endothelial cells (Figure 1). VCAM-1 was hardly detected on the carotid endothelium neither under physiologically conditions, nor after TNF $\alpha$ stimulation. ICAM-1 is physiologically present on endothelial cells, but after TNFa stimulation, a significant upregulation of ICAM-1 expression was observed ( $p$ < 0.001) (Supplementary Figure S3). Additionally, TNFa significantly increased P-selectin and PECAM-1/CD31 expression on the endothelial cells' surface. However, ICAM-1 appeared to be the most densely expressed target out of the five. For this reason, we have 
chosen ICAM-1 over the other surface adhesion ligands to conduct in vitro and ex vivo binding studies.

\section{Analysis of the binding capacity of $\mathrm{MB}_{\mathrm{ICAM}-1}$ in vitro.}

Corresponding in vitro cell stainings of TNFa-stimulated HUVEC also showed a significant increase of ICAM-1 expression (Figure 2A). The in vitro binding efficiency of ICAM-1-labeled MB was determined in customized parallel-wall flow chambers at a continuous flow of $0.25 \mathrm{ml} / \mathrm{min}\left(1.25 \mathrm{dyn} / \mathrm{cm}^{2}\right)$ on TNFa-stimulated HUVEC. The number of bound $M B$ was determined using fluorescence microscopy, showing that $\mathrm{MB}_{\mathrm{ICAM}-1}$ bound 8 times more $(p=0.0158)$ to stimulated HUVECs than to unstimulated cells (Figure 2B and E) and 14 times more than untargeted MB ( $p=0.0158$ ). There was no significant difference between the binding efficiency of non-targeted MB (Figure 2C) and targeted MB on nonstimulated HUVEC, showing the binding specificity of the MBICAM-1 under shear stress. To confirm MB binding, z-stacked images were recorded using TPLSM and reconstructed using Image Pro Analyzer. Image analysis confirmed that the MB were bound to the cellular surface and not floating on top of the cells (Figure 2D).

\section{TPLSM analysis of MB ICAM-1 $_{1}$ binding in the ex vivo carotid flow chamber assay}

To test the $\mathrm{MB}$ in a more physiological setup, we carried out further binding assays in excised inflamed murine carotid arteries (Figure $3 \mathrm{~A}$ and $\mathrm{B}$ ). Wild type mice were intraperitonially injected with $1 \mu \mathrm{g}$ TNFa and stimulated for 4 hours prior to tissue excision. We initially carried out binding studies in HBSS, which showed significant unspecific binding of untargeted control MB (Supplementary Figure S1). The untargeted MB showed an $80 \%$ retention after a 10 min washing step and $70 \%$ after a second washing step. When switching the flow medium to human whole blood, unspecific binding was reduced drastically, to $\sim 10 \%$ after two washing steps (Supplementary Figure S1). 
The ex vivo data confirmed our finding of the in vitro binding studies, showing that at a low flow rate of $0.25 \mathrm{ml} / \mathrm{min} 7.5$ times $(p=0.0304)$ more MBICAM-1 bound to TNFa-stimulated endothelium than to unstimulated endothelium. Furthermore, unspecific binding of untargeted MB was marginal, indicating ICAM-mediated binding of the MB. This was further confirmed by competition experiments with free ICAM-1 antibodies.

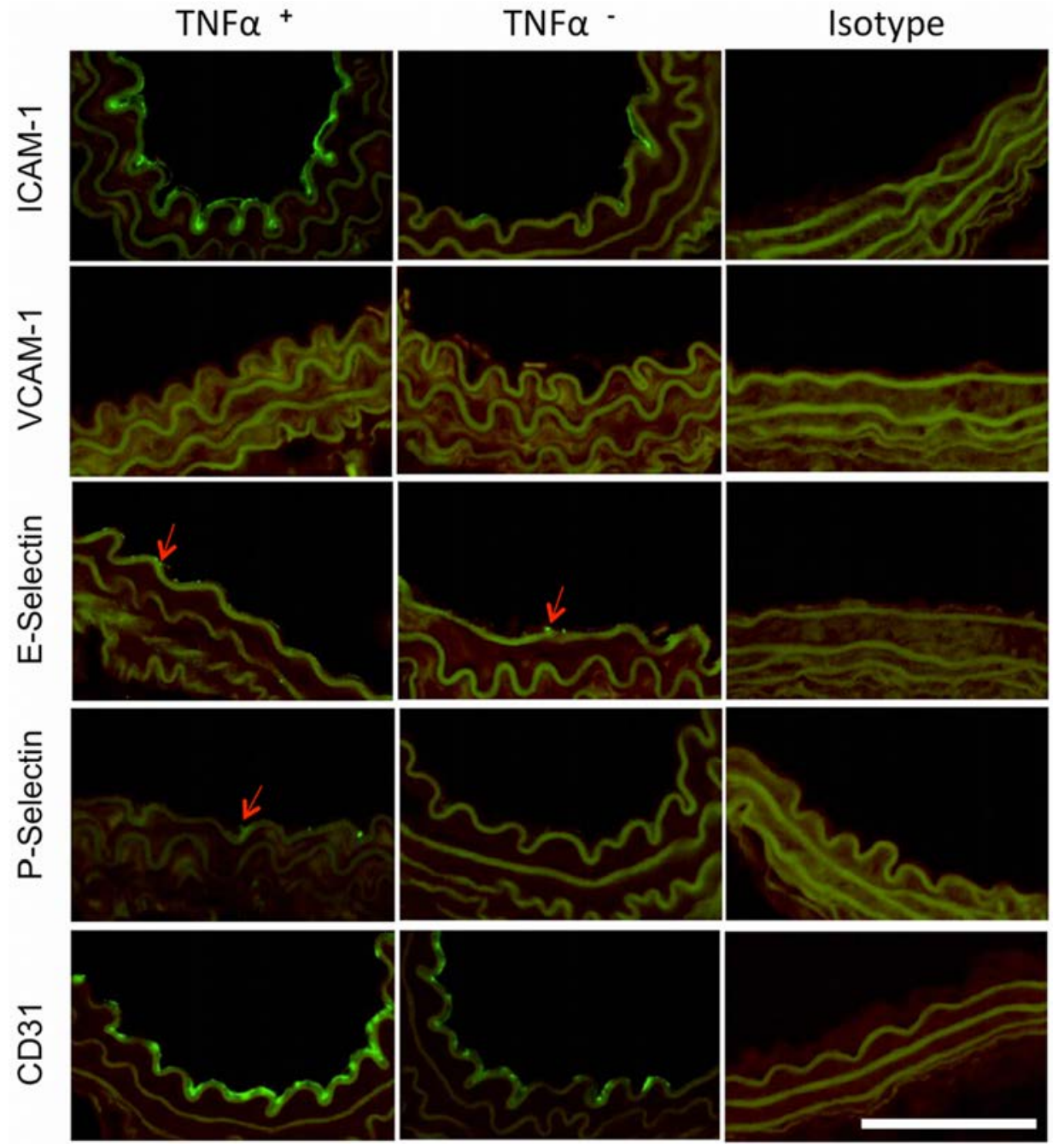

Figure 1. Immunohistochemical analysis of ICAM-1, VCAM-1, E-selectin, P-selectin and PECAM-1/CD31 expression on the luminal surface of TNFa-stimulated vs. non-stimulated carotid arteries on the endothelial luminal surface $(n=3)$. Isotype IgG antibodies were used as negative controls. Scale bar $=50 \mu \mathrm{m}$. 


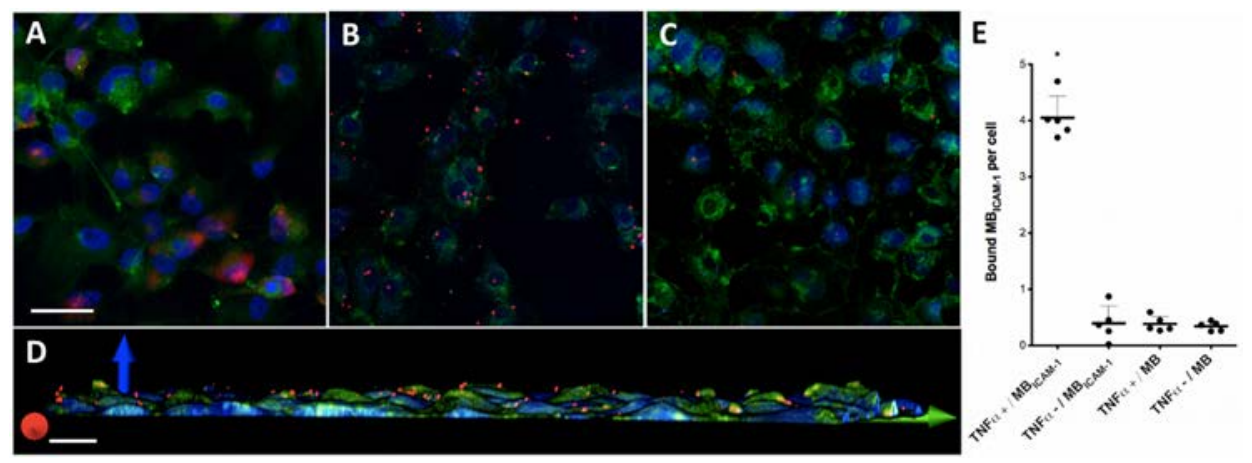

Figure 2. In vitro binding of ICAM-1-targeted rhodamine-MB to activated endothelial cells.

A) TNFa-stimulated HUVEC were stained with a primary antibody against ICAM-1 and an Alexa Fluor 568-conjugated secondary antibody (red). The cell membrane is labeled with wheat germ agglutinin Alexa Fluor 488 (green), the nucleus with SYTO 41 (blue). B) Binding of ICAM-1targeted rhodamine-MB (red) to TNFa-stimulated HUVEC. C) Binding of ICAM-1-targeted rhodamine-MB (red) to unstimulated HUVEC. D) Three-dimensional reconstruction of the zstack data exemplifying efficient binding of ICAM-1-targeted rhodamine-MB to TNFa-stimulated HUVEC. Scale bar $=50 \mu \mathrm{m}$. E) Quantitative analysis of the binding of ICAM-1-targeted and untargeted control MB to stimulated and unstimulated HUVEC. Six representative images were analyzed per in vitro binding study, and three independent bindings studies were performed. * indicates $p<0.05$ vs. all control groups.

Additionally, MB accumulation was assessed at three higher flow rates: 0.6, 0.8 and $1 \mathrm{ml} / \mathrm{min}$. While at a flow rate of $0.6 \mathrm{ml} / \mathrm{min}$, binding efficiency did not significantly decrease compared to $0.25 \mathrm{ml} / \mathrm{min}(p=0.42)$, higher flow rates i.e. $0.8 \mathrm{ml} / \mathrm{min}$ and $1 \mathrm{ml} / \mathrm{min}$ showed significantly reduced MB retention, by $38 \%$ ( $\mathrm{p}$ $=0.0304)$ and $55 \%(p=0.0304)$, respectively (Figure $3 C)$.

As shear stress is strongly dependent on flow rate and vessel diameter ${ }^{19}$, an accurate determination of vessel diameter combined with a fixed flow rate provides the corresponding shear stress for each individual carotid, accounting for variability.

For an accurate determination of shear stress in each carotid, the vessel diameters were measured based of the curvature of the blood vessel (Figure $3 \mathrm{D})^{18}$.

The MB accumulation is high at low shear stress $\left(10-50 \mathrm{dyn} / \mathrm{cm}^{2}\right)$ and decreases with increasing shear stress. 

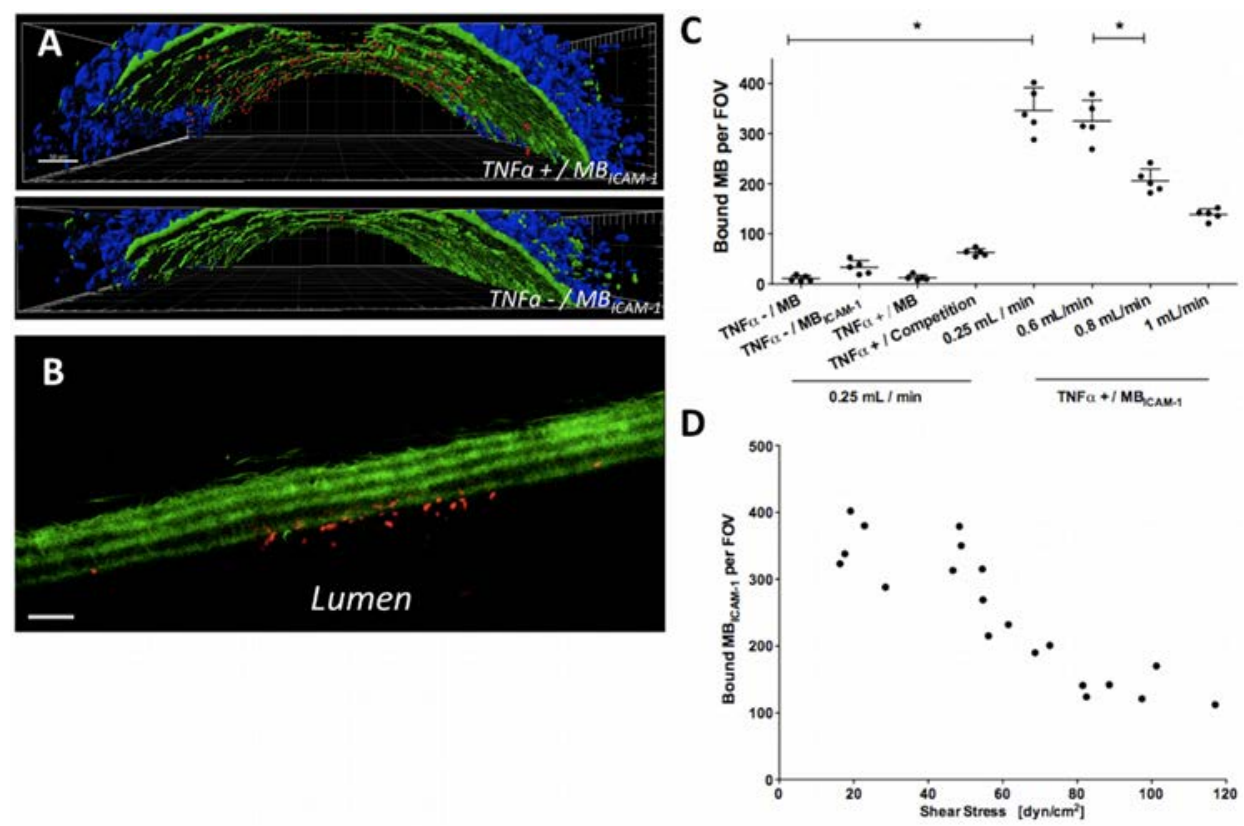

Figure 3. Ex vivo TPLSM analysis of MB binding to explanted carotids at different flow rates and shear stresses. A) Reconstructed z-stack TPLSM image exemplifying efficient binding of ICAM-1-targeted rhodamine-labeled MB (red) to a TNFa-stimulated murine carotid artery mounted in a flow chamber at a flow rate of $0.25 \mathrm{ml} / \mathrm{min}$. Collagen is shown in blue (via its second harmonic signal), elastin autofluorescence in green). B) Single plane image of the carotid wall (green) showing bound MB (red). Scale bar $=50 \mu \mathrm{m}$. Images were analyzed using Imaris. C) Quantification and statistical analysis of ( $n=5$, total of 20 animals) ex vivo binding studies at different flow rates. The numbers of bound MB per $500 \times 500 \mu \mathrm{m}$ field of view are plotted. D) Graph depicting the shear stress-dependence of ICAM-1-targeted MB binding to activated murine carotids.

\section{US analysis of $\mathrm{MB}_{\mathrm{ICAM}-1}$ binding in the ex vivo carotid artery assay}

We then investigated the binding efficiency of $\mathrm{MB}_{\text {ICAM-1 }}$ in explanted carotids mounted in flow chambers using CEUS. The gradual replacement of HBSS by blood (Figure 4A and D) could be followed in B-mode when blood cells were detected entering the field of view.

When HBSS was totally replaced by blood at $37^{\circ} \mathrm{C}$ under pulsatile injection, physiological experimental conditions were present. During continuous MB 
injection, an increase in specific signals was clearly observed in the imaging sequence of ultrasound contrast agent injection (Figure 4B and D) compared to unspecific signals detected for plain blood injection (Figure 4A).
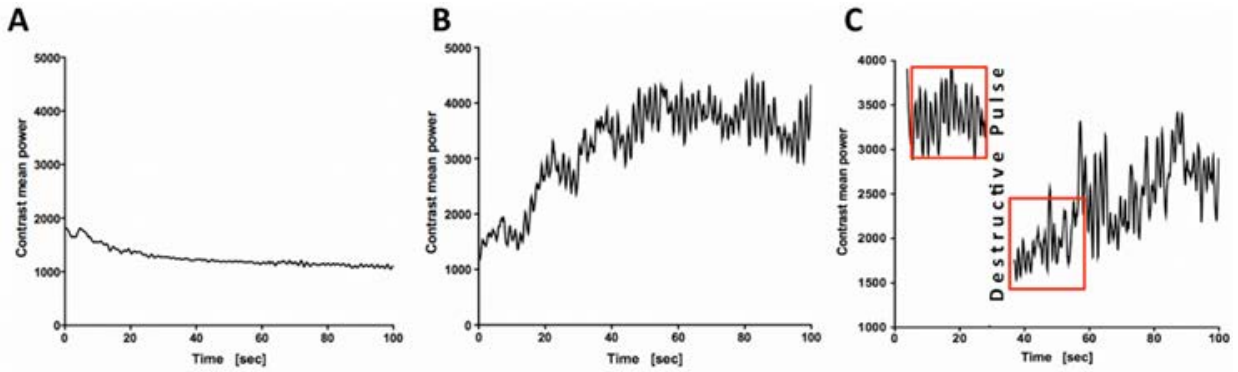

D
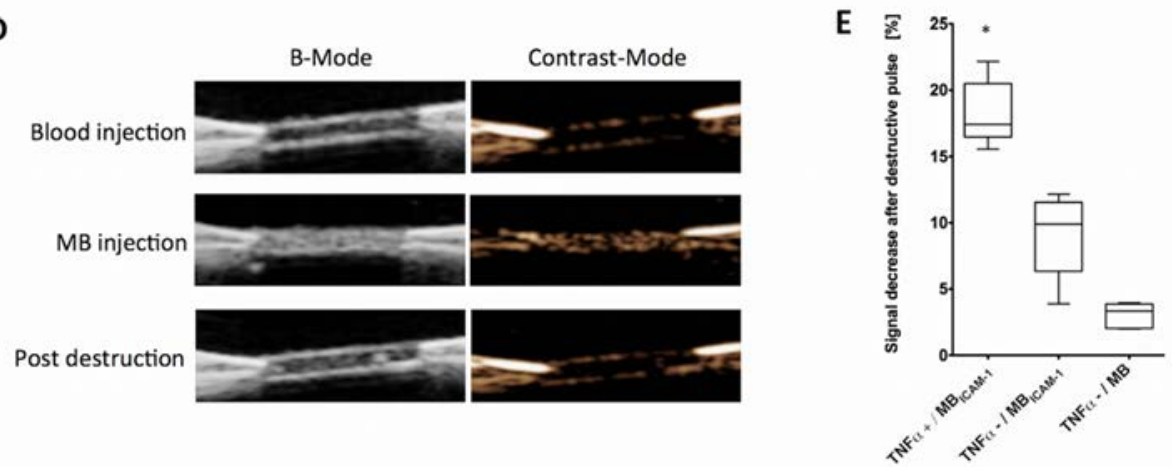

Figure 4. Representative ultrasound intensity to time curves of murine carotids ex vivo, mounted in a flow-chamber, showing that A) pure blood injection does not lead to significant changes, B) injection of ICAM-1-targeted MB leads to a rapid and strong signal enhancement and $\mathbf{C}$ ) the destruction-replenishment imaging sequence works for identifying $\mathrm{MB}_{\text {ICAM-1 }}$ binding. D) Respective CEUS images acquired using high-frequency imaging system (21 MHz; Vevo 2100, VisualSonics Inc) in B-mode and contrast-mode. E) Quantification and statistical analysis of MB binding ( $n=5$, total of 8 animals) showing specific binding of ICAM-1-targeted MB to activated carotids. * indicates $p<0.05$.

In the imaging sequence with the destructive pulse (Figure $4 C$ ), cumulative signals from bound MB together with the free-circulating ones was detected before the destruction pulse, and immediately after the destructive event only the free-circulating ones could be identified. Slow replenishment of the blood by reentering MB was seen in the same sequence. Statistical analysis of the MBICAM-1 
binding (Figure 4E) revealed a 2.5-fold higher signal decrease after $M B$ destruction ( $p=0.0158)$ in TNFa-stimulated carotids compared to the unstimulated group and a 6 -fold increase $(p=0.0158)$ compared to untargeted MB.

\section{In vivo US analysis of MB $\mathrm{MCAM}-1_{1}$ binding}

Finally, we investigated the potential of MBıсAM-1 in vivo. Similar to the ex vivo flow chamber experiments, an increase in gray scale intensity was observed during MB injection, followed by MB blood-pool distribution and subsequent clearance. While specific retention of MB was clearly visible 7 minutes after $\mathrm{MB}_{\text {ICAM-1 }}$ injection, no circulating MB and thus also no replenishment at this late time point could be observed (Figure 5). In this context, a 2.6-fold higher MB retention in TNFa stimulated carotids was found compared to unstimulated ones $(p=0.0079)$ (Figure 5C).

A

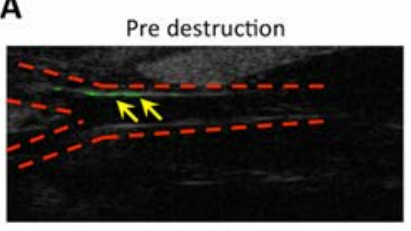

Post destruction

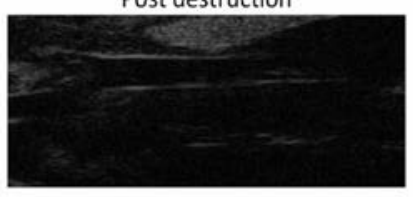

B

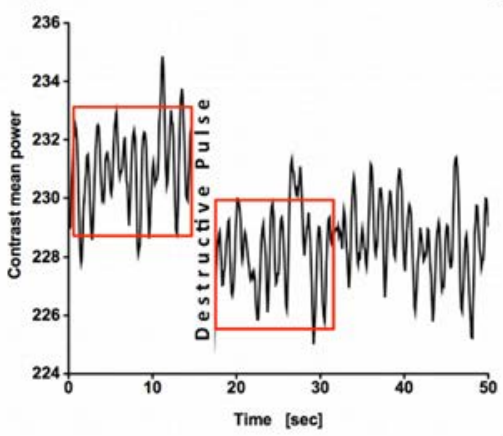

C

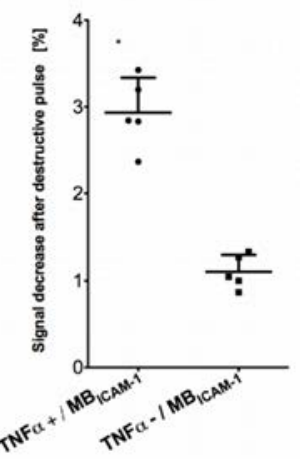

Figure 5. Representatives CEUS images of murine carotids in vivo showing A) predestructive and post-destructive CEUS images acquired using a high-frequency ultrasound imaging system (21 MHz) in contrast-mode (MB are shown in green, arrowheads), B) Example for a destruction-replenishment measurement in a mouse carotid artery in which binding of $M B_{\text {ICAM-1 }}$ to its target is analyzed. C) Quantification and statistical analysis of MB binding ( $n=5$, total of 10 animals) showing specific binding of ICAM-1-targeted MB to activated carotids. * indicates $p<0.05$. 


\section{Discussion}

In this report, we provide proof-of-principle for the complementary use of contrastenhanced ultrasound and two-photon laser scanning microscopy showing quantitative analysis of MB binding kinetics to TNFa-stimulated HUVEC in vitro, to freshly excised murine carotid arteries ex vivo and noninvasive detection in vivo.

The upregulation of ICAM-1 in response to proinflammatory stimulation has been described in previous studies in the context of atherosclerosis and is considered a primary target for in vitro and in vivo molecular imaging ${ }^{20-22}$. We identify in the present study ICAM-1 as a viable marker for early inflammation in the carotid artery with high shear stress.

The combination of TPLSM and CEUS offers a unique perspective on the MB binding. The deep-tissue submicron resolution of TPLSM offers the possibility to visualize single $\mathrm{MB}$ and consequently quantify the total number of MB bound to the endothelial surface in the field of view, whereas noninvasive CEUS imaging covers the aspect of potential clinical application. Our data shows that TPLSM quantification is in good agreement with CEUS. Both modalities were able to specifically detect bound MB on inflamed endothelium. However, a discrepancy in the factor of signal amplification after MB binding (7.5 fold (TPLSM) versus 2.5 fold (CEUS)) was observed. This may be due to an underestimation of PBCA-MB concentrations in nonlinear CEUS due to their relatively low nonlinear response. PBCA-MB are hard-shelled MB, which are known to scatter more but oscillate less than soft-shelled MB during ultrasound imaging; therefore not all bound MB may be detected nonlinearly using the bubble-specific/contrast-mode ${ }^{23}$. Using Bmode, reflection and scattering are the predominant signal sources. However, both PBCA-MB and blood cells are scatterers, while vessel walls account for reflections. This therefore results in background signals that can mask part of the scattering produced by the $\mathrm{MB}$ in $\mathrm{B}-\mathrm{mode}^{24}$. A direct correlation between the number of bound $M B$ and gray scale increase was not established, because TPLSM and CEUS were applied on separate carotids. Although such kind of a comparison would be highly interesting, it would require different levels of 
ICAM-1 expression in the carotid, which goes beyond the scope of this paper and is subject to a separate future study.

One of the key challenges of molecular imaging in major blood vessels is the retention of the contrast agent under high shear and flow rates. Nanosized contrast agents promote internalization and extravasation into the tissue ${ }^{25}$, protecting them from the shear forces in the lumen. However this effect also increases unspecific uptake, making the differentiation between marker-specific and unspecific binding challenging. MB with an average size of $2 \mu \mathrm{m}$ and a hard shell lack the flexibility of monocytes, thus they cannot extravasate and remain strictly in the vascular compartment, which makes these particles ideal endothelial contrast agents. Nevertheless, MB size and rigidness make them more prone to collisions with blood components and breaking, therefore blood flow and shear stress play a limiting factor in the retention rate of these particles. The shear stress within blood vessels is determined by many factors, such as vessel diameter, flow rate, blood viscosity and vessel architecture ${ }^{19}$. Furthermore, shear stress is not constant thoughout the entire vesse ${ }^{26}$. Not suprisingly, shear stresses found in the literature for murine carotids range between $28-142$ dyn $/ \mathrm{cm}^{227}$. However, the predominant part of previous studies determine the shear stress within murine carotid to be in the range of $36-81 \mathrm{dyn} / \mathrm{cm}^{2}$ at average flow rates between $0.35-0.75 \mathrm{ml} / \mathrm{min}^{28-32}$. In this study we show in vitro as well as ex vivo, that at low flow rate and shear stress at $0.25 \mathrm{ml} / \mathrm{min}(1.25$ dyn $/ \mathrm{cm}^{2}$ in vitro and $20 \mathrm{dyn} / \mathrm{cm}^{2}$ ex vivo), the binding of MBICAM-1 is specific to inflamed endothelium. More importantly, with increasing flow and shear stress, our data show that even at physiological shear stress conditions of 35-60 dyn/ $\mathrm{cm}^{2}$ $31,33, \mathrm{MB}$ binding is not significantly reduced. Also, our data is in line with other publications showing that at higher shear stresses MB retention and the rate of $\mathrm{MB}$ bindin ${ }^{34,35}$ can be reduced both of which explain the gradual decrease of MB accumulation in excised carotid arteries at $>60 \mathrm{dyn} / \mathrm{cm}^{2}$ shear stresses. Our in vivo data is in good agreement with the ex vivo results, confirming that ICAM-1targeted $\mathrm{MB}$ are able to bind to the inflamed vascular wall under physiological flow conditions in the carotid artery, providing comparable contrast enhancement. Ideally, Contrast-enhanced ultrasound (CEUS) would be the clinically preferred 
diagnostic tool for vascular imaging, because of its noninvasive proprieties, bedside availability, rapidity, lack of ionizing irradiation and cost-efficiency ${ }^{36}$. Polymer-based microbubbles (MB) have many features that are beneficial for in vivo targeting ${ }^{37}$. Due to their size, they remain strictly in the vascular compartment and target primarily the endothelium. Therefore, MB are highly suitable for molecular imaging of angiogenic and/or inflamed endothelial surfaces ${ }^{38}$. CEUS was successfully used in preclinical trials for tumor imaging ${ }^{39-41}$ and thrombus characterization ${ }^{42}$. Moreover, anti-VCAM-1 antibody coated MB could succesfully localize to atherosclerotic plaques in the aortic $\operatorname{arch}^{9}$. The main clinical application of ultrasound is in the field of stroke imaging. Therefore, ultrasound of the carotid artery is more clinically relevant. Furthermore, the flow and shear stress in carotid arteries are 2-3 times higher than in the aortic arch, which makes our results translatable also to the relative low flow areas such as the aortic arch.

We identify in the present study ICAM-1 as a viable marker for the early inflammation in the carotid artery, which presents a significant higher shear flow. Furthermore, as reviewed by Cheng et al. (2007), the shear stress in carotids is inversely related to the body weight and decreases from mice to humans by the factor of 6 , ranging between 9.5 and $12.4 \mathrm{dyn} / \mathrm{cm}^{2} 33$. We have shown that the MB used in this study can tolerate physiological and even much higher shear stresses in mice and therefore offer the potential for visualization of early inflammation in major blood vessel in human.

\section{Conclusion}

In this study, we have shown that CEUS using targeted MB is a valuable method to be applied for molecular imaging of carotid incipient atherosclerosis at physiological and higher shear rates. We validated and correlated the results using TPLSM, optimizing a bimodal platform for molecular imaging of rhodamineloaded MB, which is crucial for the understanding of the basic mechanisms of atherosclerosis and for the development of new strategies to reduce vessel injury and promote tissue repair, reducing the risk of life-threatening complications such as heart infarction or stroke. 


\section{Acknowledgement}

This work was supported by the core facility "Two-photon Imaging", a core facility of the Interdisciplinary Center for Clinical Research (IZKF) Aachen within the Faculty of Medicine at RWTH Aachen University.

\section{Disclosures}

None. 


\section{Supplementary figures}

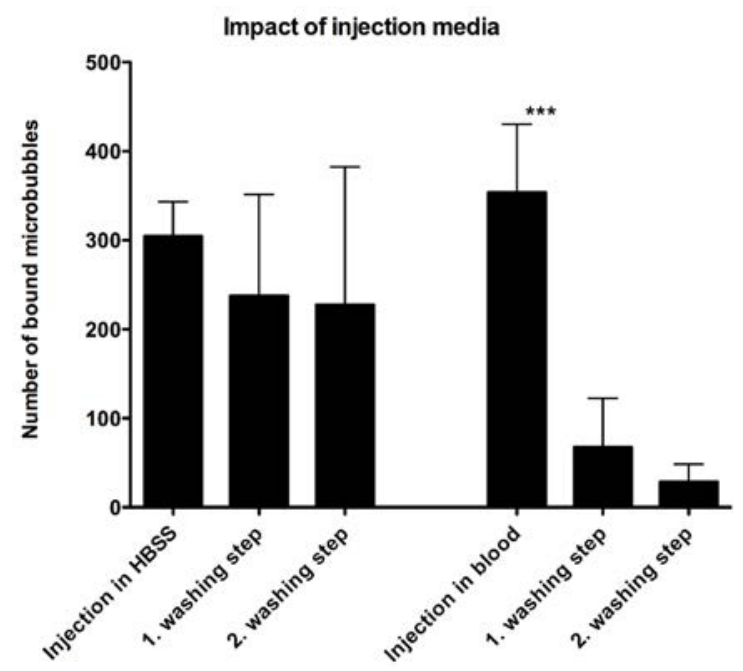

Suplementary Figure S1. Quantification and statistical analysis of unspecific MB binding in HBSS and blood $(n=3) .{ }^{* * *}$ indicates $p<0.001$ vs. both washing steps.

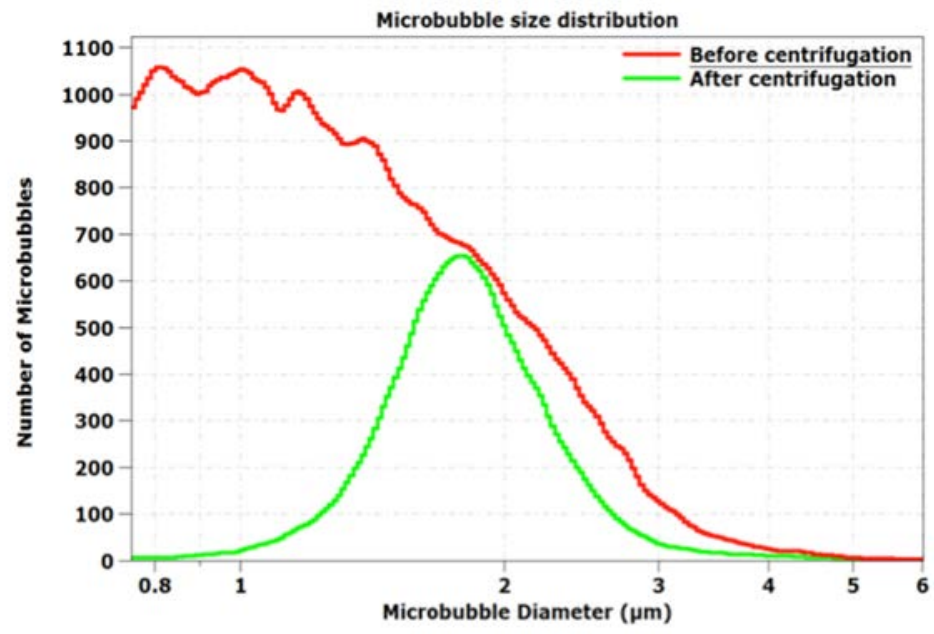

Suplementary Figure S2. Size distribution of rhodamine-loaded microbubbles before and after size isolation. 


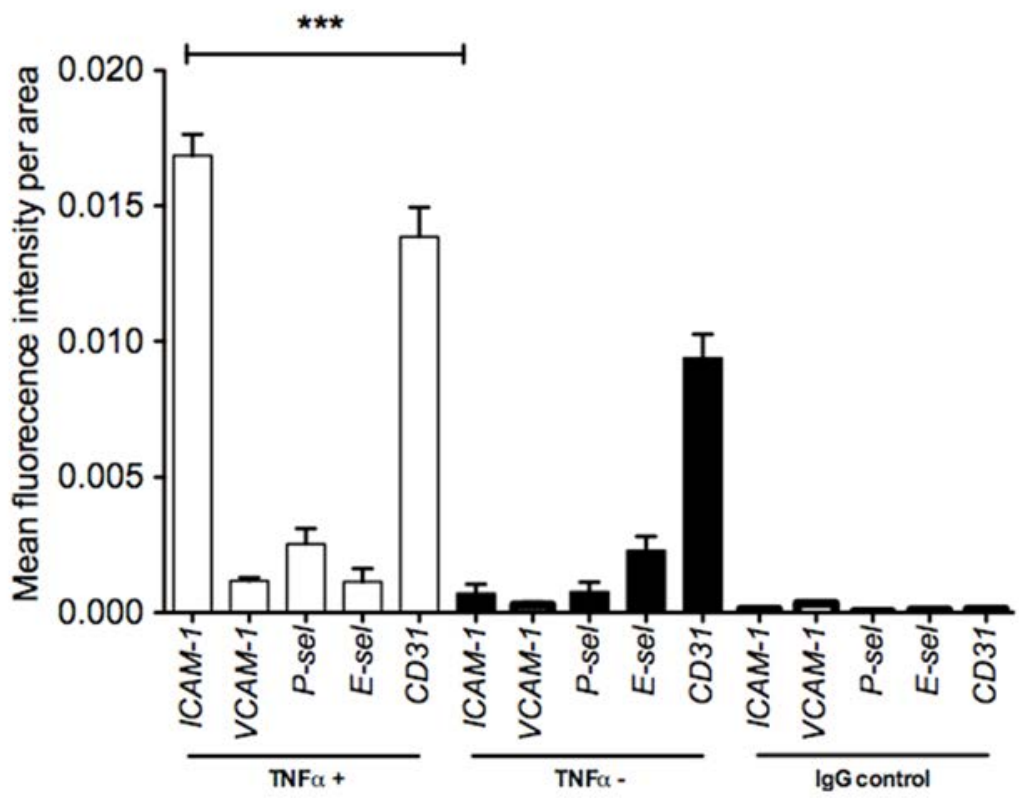

Suplementary Figure S3. Quantification of immunohistological stainings of ICAM-1, VCAM-1, P-selectin, E-selectin, PECAM-1/CD31. 


\section{References}

1. Iwata $\mathrm{H}$, Nagai R. Novel Immune Signals and Atherosclerosis. Curr Atheroscler Rep. 2012.

2. Kuhlmann MT, Cuhlmann S, Hoppe I, et al. Implantation of a carotid cuff for triggering shear-stress induced atherosclerosis in mice. J Vis Exp. 2012(59).

3. Sadeghi MM, Glover DK, Lanza GM, Fayad ZA, Johnson LL. Imaging atherosclerosis and vulnerable plaque. J Nucl Med. 2010;51 Suppl 1:51S$65 S$.

4. Libby $P$, Ridker PM, Maseri A. Inflammation and atherosclerosis. Circulation. 2002;105(9):1135-1143.

5. Lindner JR. Molecular imaging of myocardial and vascular disorders with ultrasound. JACC Cardiovasc Imaging. 2010;3(2):204-211.

6. Lindner JR. Contrast ultrasound molecular imaging of inflammation in cardiovascular disease. Cardiovasc Res. 2009;84(2):182-189.

7. Yang $\mathrm{H}$, Xiong $\mathrm{X}$, Zhang L, Wu C, Liu Y. Adhesion of bio-functionalized ultrasound microbubbles to endothelial cells by targeting to vascular cell adhesion molecule-1 under shear flow. Int J Nanomedicine. 2011;6:20432051.

8. Ferrante EA, Pickard JE, Rychak J, Klibanov A, Ley K. Dual targeting improves microbubble contrast agent adhesion to VCAM-1 and P-selectin under flow. J Control Release. 2009;140(2):100-107.

9. Kaufmann BA, Sanders JM, Davis C, et al. Molecular imaging of inflammation in atherosclerosis with targeted ultrasound detection of vascular cell adhesion molecule-1. Circulation. 2007;116(3):276-284.

10. Trachet B, Swillens A, Van Loo D, et al. The influence of aortic dimensions on calculated wall shear stress in the mouse aortic arch. Comput Methods Biomech Biomed Engin. 2009;12(5):491-499.

11. Palmowski M, Peschke P, Huppert J, et al. Molecular ultrasound imaging of early vascular response in prostate tumors irradiated with carbon ions. Neoplasia. 2009;11(9):856-863.

12. Kitagawa K, Matsumoto M, Sasaki T, et al. Involvement of ICAM-1 in the progression of atherosclerosis in APOE-knockout mice. Atherosclerosis. 
2002;160(2):305-310.

13. Sumagin R, Sarelius IH. TNF-alpha activation of arterioles and venules alters distribution and levels of ICAM-1 and affects leukocyte-endothelial cell interactions. Am J Physiol Heart Circ Physiol. 2006;291(5):H2116-2125.

14. Koczera P, Wu Z, Fokong S, et al. Fluorescently labeled microbubbles for facilitating translational molecular ultrasound studies. Drug Delivery and Translational Research. 2012;2(1):56-64.

15. Fokong S, Siepmann M, Liu Z, Schmitz G, Kiessling F, Gatjens J. Advanced characterization and refinement of poly $\mathrm{N}$-butyl cyanoacrylate microbubbles for ultrasound imaging. Ultrasound in medicine \& biology. 2011;37(10):16221634.

16. Megens RT, Reitsma S, Schiffers PH, et al. Two-photon microscopy of vital murine elastic and muscular arteries. Combined structural and functional imaging with subcellular resolution. J Vasc Res. 2007;44(2):87-98.

17. Kiessling F, Bzyl J, Fokong S, Siepmann M, Schmitz G, Palmowski M. Targeted ultrasound imaging of cancer: an emerging technology on its way to clinics. Curr Pharm Des. 2012;18(15):2184-2199.

18. van Triest HJ, Megens RT, van Assen HC, ter Haar Romeny BM, van Zandvoort MA. Arterial radius estimation from microscopic data using a new algorithm for circle parameter estimation. J Biomed Opt. 2010;15(2):026012.

19. Papaioannou TG, Stefanadis C. Vascular wall shear stress: basic principles and methods. Hellenic J Cardiol. 2005;46(1):9-15.

20. Danila D, Partha R, Elrod DB, Lackey M, Casscells SW, Conyers JL. Antibody-labeled liposomes for CT imaging of atherosclerotic plaques: in vitro investigation of an anti-ICAM antibody-labeled liposome containing iohexol for molecular imaging of atherosclerotic plaques via computed tomography. Texas Heart Institute journal / from the Texas Heart Institute of St. Luke's Episcopal Hospital, Texas Children's Hospital. 2009;36(5):393403.

21. Rautou PE, Leroyer AS, Ramkhelawon B, et al. Microparticles from human atherosclerotic plaques promote endothelial ICAM-1-dependent monocyte adhesion and transendothelial migration. Circ Res. 2011;108(3):335-343. 
22. Demos SM, Alkan-Onyuksel H, Kane BJ, et al. In vivo targeting of acoustically reflective liposomes for intravascular and transvascular ultrasonic enhancement. J Am Coll Cardiol. 1999;33(3):867-875.

23. Fokong S, Siepmann M, Liu Z, Schmitz G, Kiessling F, Gatjens J. Advanced characterization and refinement of poly N-butyl cyanoacrylate microbubbles for ultrasound imaging. Ultrasound Med Biol. 2011;37(10):1622-1634.

24. Davros WJ, Zagzebski JA, Madsen EL. Frequency-dependent angular scattering of ultrasound by tissue-mimicking materials and excised tissue. J Acoust Soc Am. 1986;80(1):229-237.

25. Yu MK, Park J, Jon S. Targeting strategies for multifunctional nanoparticles in cancer imaging and therapy. Theranostics. 2012;2(1):3-44.

26. Shaaban AM, Duerinckx AJ. Wall shear stress and early atherosclerosis: a review. AJR Am J Roentgenol. 2000;174(6):1657-1665.

27. Lammeren Wv, F LM, Borst GJ, de Kleijn DP, JP PMdV, Pasterkamp G. Atherosclerotic plaque biomarkers: beyond the horizon of the vulnerable plaque. Curr Cardiol Rev. 2011;7(1):22-27.

28. Hartley CJ, Reddy AK, Madala S, et al. Hemodynamic changes in apolipoprotein E-knockout mice. Am J Physiol Heart Circ Physiol. 2000;279(5):H2326-2334.

29. Li YH, Reddy AK, Taffet GE, Michael LH, Entman ML, Hartley CJ. Doppler evaluation of peripheral vascular adaptations to transverse aortic banding in mice. Ultrasound Med Biol. 2003;29(9):1281-1289.

30. Korshunov VA, Berk BC. Flow-induced vascular remodeling in the mouse: a model for carotid intima-media thickening. Arterioscler Thromb Vasc Biol. 2003;23(12):2185-2191.

31. Castier Y, Brandes RP, Leseche G, Tedgui A, Lehoux S. p47phoxdependent NADPH oxidase regulates flow-induced vascular remodeling. Circ Res. 2005;97(6):533-540.

32. Rudic RD, Bucci M, Fulton D, Segal SS, Sessa WC. Temporal events underlying arterial remodeling after chronic flow reduction in mice: correlation of structural changes with a deficit in basal nitric oxide synthesis. Circ Res. 2000;86(11):1160-1166. 
33. Cheng C, Helderman F, Tempel D, et al. Large variations in absolute wall shear stress levels within one species and between species. Atherosclerosis. 2007;195(2):225-235.

34. Takalkar AM, Klibanov AL, Rychak JJ, Lindner JR, Ley K. Binding and detachment dynamics of microbubbles targeted to P-selectin under controlled shear flow. J Control Release. 2004;96(3):473-482.

35. Wu J, Leong-Poi $\mathrm{H}$, Bin J, et al. Efficacy of contrast-enhanced US and magnetic microbubbles targeted to vascular cell adhesion molecule-1 for molecular imaging of atherosclerosis. Radiology. 2011;260(2):463-471.

36. Kiessling F, Gaetjens J, Palmowski M. Application of molecular ultrasound for imaging integrin expression. Theranostics. 2011;1:127-134.

37. Kiessling F, Fokong S, Koczera P, Lederle W, Lammers T. Ultrasound microbubbles for molecular diagnosis, therapy, and theranostics. J Nucl Med. 2012;53(3):345-348.

38. Partovi S, Loebe M, Aschwanden M, et al. Contrast-enhanced ultrasound for assessing carotid atherosclerotic plaque lesions. AJR Am J Roentgenol. 2012;198(1):W13-19.

39. Bzyl J, Palmowski M, Rix A, et al. The high angiogenic activity in very early breast cancer enables reliable imaging with VEGFR2-targeted microbubbles (BR55). Eur Radiol. 2012.

40. Bzyl J, Lederle W, Rix A, et al. Molecular and functional ultrasound imaging in differently aggressive breast cancer xenografts using two novel ultrasound contrast agents (BR55 and BR38). Eur Radiol. 2011;21(9):1988-1995.

41. Rix A, Lederle W, Siepmann $M$, et al. Evaluation of high frequency ultrasound methods and contrast agents for characterising tumor response to antiangiogenic treatment. Eur J Radiol. 2012;81(10):2710-2716.

42. Schumann PA, Christiansen JP, Quigley RM, et al. Targeted-microbubble binding selectively to GPIIb IIla receptors of platelet thrombi. Invest Radiol. 2002;37(11):587-593. 



\section{Chapter 3}

\section{Induction of accelerated}

atherosclerosis in mice: the "wire-injury" model

Adelina Curaj, Zhuojun Wu, Mareike Staudt, Elisa A. Liehn

Accepted for publication in JoVE 


\section{Abstract}

Atherosclerosis is a proliferative fibro-inflammatory disease developing in the arterial wall, inducing a deficient blood flow or a lack of blood flow. Moreover, by rupture of the defective vascular wall, atherosclerosis induces occlusive thrombus formation, which represents the main cause of myocardial infarction or stroke and the most frequent cause of death. Despite the advances in the cardiovascular field, many questions remain unanswered, and additional basic research is essential to improve our understanding of the molecular mechanisms during atherosclerosis and its effects. Due to limited clinical studies, there is a need for representative animal models recreating atherosclerotic conditions such as neointima formation after stent implantation, balloon angioplasty, or endarterectomy. Since the mouse presents many advantages and is the most frequently used model for studying molecular processes, the current study proposes an invasive procedure of endothelial denudation, also known as the wire-injury model, which is representative of the human condition of neointima formation in arteries after revascularization procedures. 


\section{Introduction}

Atherosclerosis is the main pathology underlying cardiovascular events such as myocardial infarction or stroke. The main mechanisms triggering acute cardiovascular syndromes are plaque rupture, superficial erosion, and thrombus formation. There are multiple clinical situations connected to the plaque development: native atherosclerotic plaque, restenosis after endarterectomy, and restenosis after balloon angioplasty with/without stent implantation ${ }^{1}$. After arterial injury, suppression of the inflammatory processes ${ }^{2,3}$ and the recovery of the endothelial compartment are essential to prevent further complications ${ }^{1}$. Clinical research is limited to tissue and blood samples due to ethical considerations, costs, and a lack of knowledge in basic mechanisms. For these reasons, there is a need to study molecular mechanisms in animal models ${ }^{4-6}$, which can recreate the clinical conditions. Our model of accelerated neointima formation in the context of atherosclerosis is the result of many years of experience in the implementation of these models in small animals ${ }^{7-11}$. The mouse model is the most attractive model for research, due to its ease of handling, the ability to have large animal groups due to low costs related to animal purchase and care, and the availability of various transgenic and knockout strains.

The major disadvantage of the mouse model is the small size of the main arteries subjected to atherosclerotic disease (the carotid artery, the aorta, and the femoral artery), which requires qualified surgical expertise and skills to manipulate the vessels and to invasively induce an atherosclerotic plaque. Therefore, the model of accelerated neointima formation, in the context of restenosis after endarterectomy or stent implantation, proposed in this paper is presented with a step-by-step guideline and suggestions to ease the introduction for interested personnel. Another disadvantage is that the denudation is made on the normal arterial wall, and therefore, the neointima formation will be moderate compared with the clinical situation. The high level of plasma cholesterol reached in apolipoprotein $\mathrm{E}$ knockout $\left(A p o E^{--}\right)$mice fed with a high fat-diet creates a proper pro-inflammatory environment needed for the neointima formation. 
The surgery is performed under a stereomicroscope. The carotid artery is exposed by a median incision in the ventral cervical area. Anatomical structures on top of and surrounding the carotid artery are minimally manipulated to reduce post-surgical inflammation. The carotid artery bifurcation is exposed. To induce accelerated neointima formation, internal and external carotid arteries are prepared for blood flow cessation and subsequent common carotid artery denudation. In conclusion, the method can be learned by personnel with minimal experience in animal surgeries.

\section{Protocol}

Experiments presented in this paper are performed according to the German law and to the European animal care guidelines. The animals are bred in the animal facility of the Institute for Laboratory Animal Science, University Hospital Aachen, Germany, under supervision of Prof. Dr. R. Tolba and Dr. A. Teubner (animal welfare officer).

\section{1) Animal care}

Keep the mice in a specialized care unit, assuring proper access to food and specialized veterinary control and treatment. If the animals are moved or purchased from third parties, please assure a one-week accommodation period before undergoing the procedure.

\section{2) Hyperlipidemia inducement}

Feed 6-8 weeks old, 18-20 g, female (optional) ApoE-/- mice with an atherogenic diet ( $21 \%$ fat, $0.15 \%$ cholesterol, $19.5 \%$ casein, wt/wt) one week prior to the surgical procedure and continue the diet until the atherosclerotic plaque analysis is to be performed. 


\section{3) Surgical preparation}

3.1) Anesthetize the mice using intraperitoneal injection of $100 \mathrm{mg} / \mathrm{kg}$ bodyweight ketamine and $10 \mathrm{mg} / \mathrm{kg}$ bodyweight xylazine. Confirm proper anesthetization prior to surgery by the lack of reflexes and whisker movement. Cover the mouse eyes by a film of eye cream, to prevent eye-dryness and blindness during surgery.

3.2) Assure the maintenance of sterile conditions to avoid infections during surgery by using sterile materials and instruments.

3.3) Shave the mice in the ventral neck region. Disinfect the skin with betadine prior to incision. Make a skin incision of $1 \mathrm{~cm}$ in the median region of the neck area, on top of the trachea.

3.4) Separate the two fat bodies to ensure a proper view over the tracheal region. Use retractors to hold the muscle layer and expose the carotid artery. If present, perform the blunt-dissection of the thin muscle layer covering the carotid artery.

3.5) Use a sharp curved forceps to separate the carotid artery from the vagus nerve and jugular vein. Thus, the bifurcation area with the internal and external carotid artery should be visible. Use $\mathrm{NaCl} 0.9 \%$ in order to avoid tissue dryness during the surgical procedure.

\section{4) Wire-injury}

4.1) Place a $7 \mathrm{~cm}$ long $0 / 5$ silk suture under the carotid artery, proximal to the aortic arch. Make an open loop, ready to be closed anytime.

4.2) Place two $0 / 7$ silk sutures (each $1.5 \mathrm{~cm}$ long) around the external carotid artery: one loop close to the bifurcation point, and one loop as distal as possible. Prepare them as an open loop, ready to be closed anytime.

4.3) Place one $0 / 7$ silk suture $(1.5 \mathrm{~cm}$ long $)$ under the internal carotid artery. Prepare it as an open loop, ready to be closed anytime.

4.4) Position the mouse-table with the mouse-head towards the operator to 
assure proper position for the guide-wire insertion during the denudation (Figure 1A).

4.5) Under microscopic view, stop the blood flow through the common carotid artery by holding and pulling the ends of the $0 / 5$ silk suture with a hemostat forceps.

4.6) Immediately after common carotid artery ligature, close the suture loops placed on the internal carotid artery and the distal suture on the external carotid artery tightly (Figure 1B).

4.7) Perform a small incision (arteriotomy, half of the vessel diameter) distal in the external carotid artery, between the two loops, using the small scissors (Figure 1C). If the incision is too big, please follow the troubleshooting instructions.

4.8) Prepare a 0.14 -inch polished flexible guide-wire by disinfection with alcohol and moisten it in a droplet of sodium chloride to assure proper sliding into the vessel.

4.9) Insert the guide-wire into the common carotid artery via the transverse arteriotomy of the external carotid artery (Figure 1D). Obtain endothelial denudation by passing the guide-wire along the vessel while rotating and repeat this procedure for three times. It is important to maintain the same amplitude of rotational movement in each mouse to increase the reproducibility.

4.10) Close the proximal loop on the external carotid artery tightly. Restore the blood flow in the carotid artery by cutting the suture around common artery and the suture around the internal carotid artery. 
A

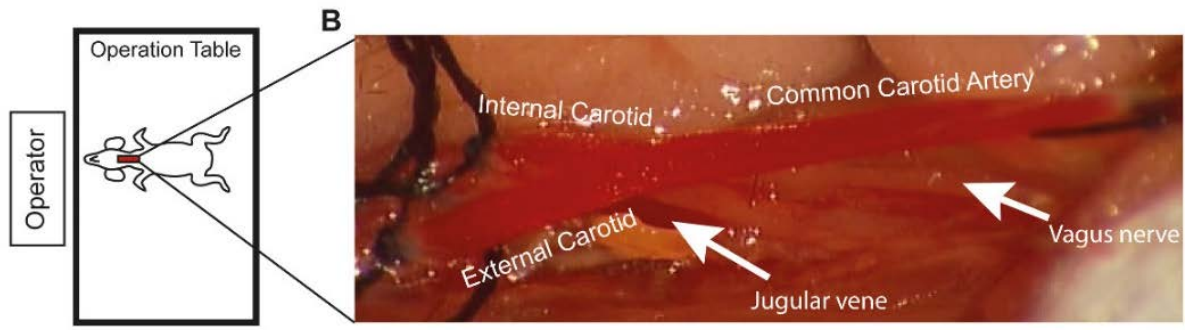

C

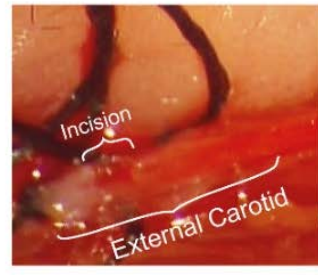

D

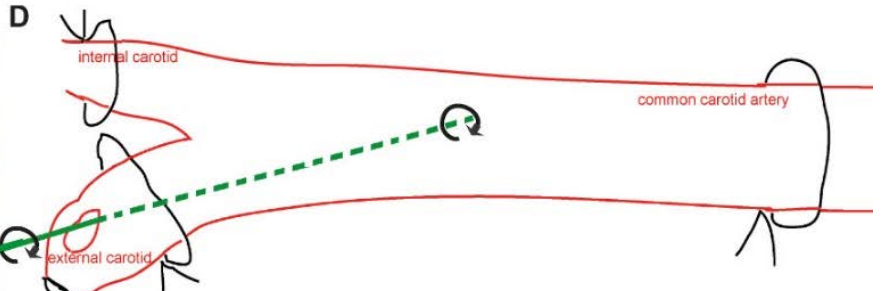

Figure 1. Schematic representation of operative procedure. A) The positioning of the operation table toward the operator during the wire-injury procedure. B) Enlarged view of the common carotid artery and its branches, as it appears under the microscope. C) The size of the incision in the external carotid artery. D) Schematic representation of wire-injury procedure using the 14" guide-wire.

\section{5) Suture and recovery}

5.1) Remove the retractors and bring back the muscle layer and the two fat bodies to the physiological position.

5.2) Close the skin with 3 separated sutures $0 / 6$, if echocardiographic measurements are needed. If no imaging is needed, use metallic clips to close the skin.

5.3) Lay down the mouse on the left side under the infrared until it wakes up. Do not leave an animal unattended and in the company of other animals until fully recovered.

5.4) For future identification, mark the mouse using the local system (ask the animal welfare officer from the institution). 


\section{6) Analysis of the atherosclerotic plaque}

6.1) Anesthetize the mice at the end-time point using intraperitoneal injection of $100 \mathrm{mg} / \mathrm{kg}$ bodyweight ketamine and $10 \mathrm{mg} / \mathrm{kg}$ bodyweight xylazine. Confirm proper anesthetization by the lack of reflexes and feelers movement.

6.2) Perform exsanguination by retro-orbital or cardiac puncture and collect the blood for further analysis².

6.3) Disinfect the skin with betadine. Open the thoracic cavity and remove the right auriculum of the heart. Perfuse phosphate buffered solution through left ventricle to remove the remaining blood from the vessel and then perfuse $4 \%$ PFA to fix the tissue. If no fixation is required, explant the carotid artery immediately after washing ${ }^{2,4,11}$. Perform the usual protocols with analyses of interest: paraffin embedding, cryosection, mRNA or protein analysis, etc.

6.4) For morphometrical measurements, explant carefully, with minimal manipulation, the carotid artery including the bifurcation as proximal to the aortic arch using curved forceps and small scissors.

6.5) Embed the carotid artery in the paraffin block using routine embedding protocols. To perform transversal sectioning, place the carotid artery upright on bifurcation. Cut serial $5 \mu \mathrm{m}$ thick serial sections starting with the bifurcation and collect them all on coated histological slides. (Figure 2A).

6.6) Stain every $10^{\text {th }}$ section using Movat staining for highlighting of laminas ${ }^{2,4,11}$. After collecting microscopic pictures of all vessels (usually using 10x objective), measure the lumen, internal and external lamina for each section, using special designed software (DISKUS, Hilgers, Germany) ${ }^{2,4,11}$, as shown in Figure 2B and calculate the intimal growth and media of the vessels.

6.7) Analyze smooth muscle cells and macrophage content, or endothelial recovery in serial section using usual immunohistological staining ${ }^{2}$ (Figure $2 \mathrm{C}$ ). 
A

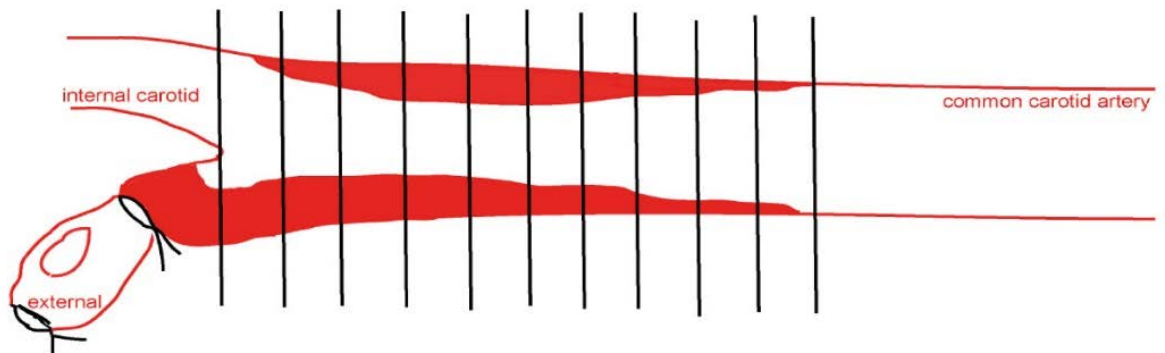

Intima $=$

B
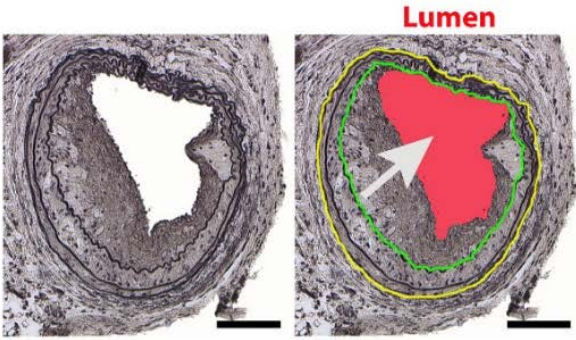

Lam. int. - Lumen

Media $=$

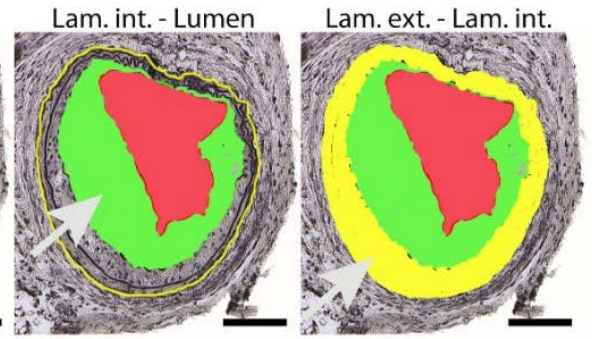

C
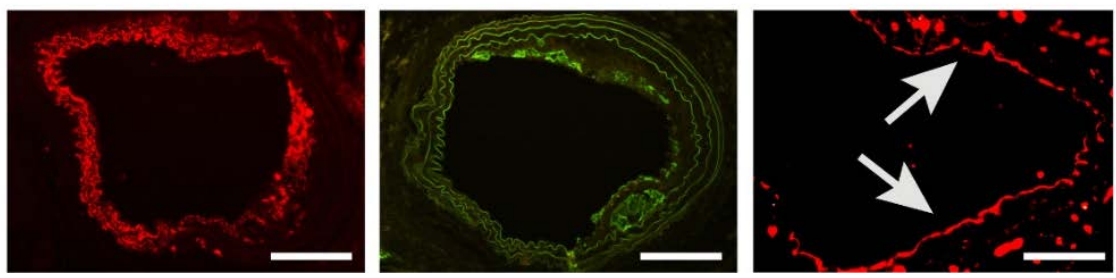

Figure 2. Analysis of Restenosis Plaque. A) Schematic representation of plaque analysis in the common carotid artery, 4 weeks after wire-injury induction B) Neointima formation 4 weeks after the wire-injury and schematic representation of main parameters used for analysis. Intima (green area) is the difference between the lumen (red) and the lamina interna (green line). Media (yellow area) is the difference between the lamina externa (yellow line) and interna (green line). Scale bar $100 \mu \mathrm{m} \mathrm{C)} \mathrm{Representative} \mathrm{images} \mathrm{of} \mathrm{the} \mathrm{staining} \mathrm{of} \mathrm{the} \mathrm{main} \mathrm{cell} \mathrm{types} \mathrm{involved} \mathrm{in} \mathrm{neointima}$ formation. Smooth muscle cells (smooth muscle actin -red, scale bar $100 \mu \mathrm{m}$ ), macrophages (Mac 2green, scale bar $100 \mu \mathrm{m}$ ) and endothelial cells (CD31- red, arrows, scale bar $50 \mu \mathrm{m}$ ).

\section{Representative results}

The atherosclerotic plaque induction procedure takes 15 - 20 minutes and shows a minimal mortality rate, mostly due to the bleeding occurring during the procedure. After surgery, the mice recover from anesthesia within 20 - 25 
minutes. No physical impairment, such as paralysis, or feeding disturbance was observed after the surgery.

The wire-injury induces a de-endothelialization, mimicking vascular lesions after balloon denudation or stent-implantation. Immediately after injury, the denuded vascular wall will be covered with a layer of thrombocytes, which mediates and favors the adhesion of the monocytes ${ }^{12}$. Activated smooth muscle cells from the media will proliferate and migrate into the intimal spaces, forming the neointima. Other progenitors for smooth muscle cells will migrate from the blood (estimated to be $40 \%$ ) and contribute to the neointima growth. The plaque formation will end after the complete reendothelialization, usually 4 weeks after the wire-injury.

The neointima formation can be assessed using Movat staining.

The plaque size is calculated for each slide using software as shown in Figure 2B. The total plaque size (left carotid artery) can vary between 70,000 - 100,000 $\mu \mathrm{m}^{2}$, while the control vessel size (right carotid artery) can vary between 7,000 $8,000 \mu \mathrm{m}^{2}$. These values depend largely on the surgeon. Therefore, we strongly recommend using the same surgeon during the experiments for the same study.

The developed plaque resembles in stent restenosis, which predominately consists of proliferated and migrated smooth muscle cells from the media. The cellular composition determined by immunological staining procedures shows that the smooth muscle cell content is approximately $30-40 \%$, while macrophages are found in $15-25 \%$ of the neointima of the injured vessel. The reendothelialization can be measured after staining for an endothelial marker, and calculated as the percentage of circumference stained over the entire circumference of the lumen. Usually the reendothelialization reaches $80-90 \%$ after 3 weeks, and should almost be complete after 4 weeks (Figure 2C). To track the plaque growth during its development, the same analysis can be repeated for every time-point after the wire-injury, depending on the interest and the subject studied (see Table 1). 
Table 1. Time-dependent Plaque's Development.

\begin{tabular}{|l|l|l|l|l|l|}
\hline Time & Thrombus & $\begin{array}{l}\text { Plaque } \\
\left(\boldsymbol{\mu m}^{2}\right)\end{array}$ & $\begin{array}{l}\text { Macrophages } \\
(\% \text { from } \\
\text { Plaque })\end{array}$ & $\begin{array}{l}\text { Smooth muscle } \\
\text { cells } \\
(\% \text { from } \\
\text { Plaque })\end{array}$ & $\begin{array}{l}\text { Reendothelialization } \\
(\% \text { lumen } \\
\text { circumference })\end{array}$ \\
\hline 1 day & present & 0 & 0 & 0 & 0 \\
\hline 1 week & - & $<30000$ & $>10$ & $<50$ & $<50$ \\
\hline 2 weeks & - & $<50000$ & $>10$ & $<50$ & $>50$ \\
\hline 3 weeks & - & $<70000$ & $15-25$ & $30-40$ & $80-90$ \\
\hline 4 weeks & & $\begin{array}{l}70000- \\
100000\end{array}$ & $15-25$ & $30-40$ & complete \\
\hline
\end{tabular}

\section{Discussion}

In this paper, we provide useful tips to perform the wire-injury procedure even by personnel with minimal experience in animal surgeries. There are two critical steps in performing this procedure: the incision of the external carotid artery and the insertion of the wire. The incision in the external carotid artery needs to be performed as far as possible from the bifurcation, in order to ensure enough remaining material (Figure $1 \mathrm{C}$ ). The incision should not be too large, due to the risk of cutting the entire vessel. The second critical step is the high risk of bleeding during the arteriotomy and the insertion of the guide wire if blood flow is not efficiently discontinued. Moreover, endothelial denudation might not take place or arterial rupture is possible if the guide wire is not properly introduced in the lumen vessel. To avoid this, the surface of the guide wire must be carefully polished before the operation.

To optimize the protocol, the position of the operating-table with the mouse-head towards the surgeon ensures a better view, accessibility and control for the proper guide wire manipulation. Moreover, to increase the reproducibility, use the same guide wire in all of the studies. Since the wire size does not change, it is important to consider and eliminate all the possible differences between the mice by using the same gender, age and weight for all mice included in a study. Thereafter, Evans-Blue staining will help the surgeon determine the efficiency of the denudation. The existence of appropriate equipment is a prerequisite for the 
success of the procedure. A 10X stereomicroscope is essential for performing this procedure. The proper preparation of the guide-wire (for example polishing it) is crucial. Therefore, we strongly recommend that the guide wire preparation be performed by specialized technical personnel where available.

There are many troubleshooting steps in this protocol. If incising the external carotid artery near the bifurcation, carefully bind the externa, near the bifurcation, so no bleeding occurs. During cutting, the external carotid artery cannot be seen. Therefore, consider the bifurcation at the level of silk suture. Collect sections when the silk suture disappears. If the incision in the external carotid artery is too large and the vessel is ruptured, ensure that the blood flow into the carotid communis and internal carotid artery is effectively interrupted and try to find the opening of the vessel using forceps. After introducing the guide wire and performing the denudation, bind the vessel near the bifurcation. During cutting, start to collect when the silk from the suture starts to disappear. If arterial rupture occurs during the denudation with the guide wire, check under the microscope if the guide wire is properly polished.

Despite the similarity of the wire-injury model to clinical situations, many groups are focused on native atherosclerosis in mice, or they choose invasive atherosclerosis inductions, such as balloon angioplasty in rats or rabbits, because of the lack of trained personnel who can perform small animal surgeries. Despite the benefits of using rabbits/rats, e.g. no need for miniaturized equipment, neither rat models nor rabbit models offer a variety of different knockout strains, in terms of studying molecular mechanisms involved in neointima growth and in-stent thrombosis.

The existing models for studying in-stent restenosis in mice are difficult, require high surgical skills, and have high risks of complications such as bleeding or paralysis. For example, the mechanical injury or stent-implantation into the thoracic aorta via femoral artery is accompanied by a high mortality rate $(35 \%)$ due to hind leg paralysis or bleeding ${ }^{13-15}$. We also describe stent implantation in the carotid artery of a mouse ${ }^{16}$. The procedure is similar; however, the tissue processing for analysis is complicated and is not available to all laboratories ${ }^{16}$. 
The carotid artery is directly accessible, not only for operation procedures, but also for existing imaging methods such as ultrasound imaging. Other injury inductions in the carotid arteries in mice can be done using electrical devices ${ }^{17}$. This method is easy to perform and ensures high reproducibility. However, it induces injury in all vessel layers, which is not identical with mechanical injury. Balloon applications have benefits, e.g. the adjustment to the vessel diameter in line with the clinical practice and has strong influence on the pathological outcome. Even though mouse balloons are available, they are very expensive and therefore, not widely used. Instead, the wire-injury is the established method, mimicking in-stent stenosis.

The denudation is performed on the normal arterial wall, though with an atherosclerotic background. Therefore, the neointima formation will be moderate compared to the clinical situation. The high number of preclinical models demonstrates that none of the models fulfill all of the criteria necessary to uncover the entirety of the cellular and molecular mechanisms leading to the pathophysiology in humans (see Table 2).

After performing the wire-injury procedure, other biological and molecular analysis can be performed to identify cells, proteins, mRNAs, microRNAs, genes or other biomarkers, which can be used as therapeutic targets to develop new treatment strategies for atherosclerosis, and in particular for neointima formation after vascular injury. If available, the plaque growth can be monitored using high frequency ultrasound or other high-resolution imaging techniques. Moreover, mastering this technique would give the operator the opportunity to adapt the protocol to other invasive atherosclerosis inducement models, such as collar placement, partial ligation or even stent implantation. 
Table 2. Advantages and Disadvantages of Existent Models of Arterial Injury.

\begin{tabular}{|c|c|c|c|}
\hline Model & Animals & Advantages & Disadvantages \\
\hline \multirow[t]{2}{*}{$\begin{array}{l}\text { Diet-induced native } \\
\text { atherosclerosis }\end{array}$} & Small & $\begin{array}{l}\text { mimics the atherosclerosis } \\
\text { pathology; } \\
\text { ease of handling; } \\
\text { no surgery; } \\
\text { no stress for the animals; } \\
\text { low costs related to animal } \\
\text { purchase and care; } \\
\text { availability of various } \\
\text { transgenic and knockout } \\
\text { strains. }\end{array}$ & $\begin{array}{l}\text { low reproductibility; } \\
\text { high variance; } \\
\text { increased animal number } \\
\text { required; } \\
\text { increased waiting time. }\end{array}$ \\
\hline & Big & $\begin{array}{l}\text { mimics the atherosclerosis } \\
\text { pathology; } \\
\text { ease of handling; } \\
\text { no surgery; } \\
\text { no stress for the animals. }\end{array}$ & $\begin{array}{l}\text { low reproducibility; } \\
\text { high variance; } \\
\text { increased animal number } \\
\text { required. }\end{array}$ \\
\hline \multirow[t]{2}{*}{ Balloon dilatation } & Small & $\begin{array}{l}\text { mimics restenosis after } \\
\text { balloon angioplasty; } \\
\text { low costs related to animal } \\
\text { purchase and care; } \\
\text { availability of various } \\
\text { transgenic and knockout } \\
\text { strains. }\end{array}$ & $\begin{array}{l}\text { small size of the main } \\
\text { arteries; } \\
\text { requires qualified surgical } \\
\text { expertise; } \\
\text { balloons very expensive } \\
\text { denudation is made on } \\
\text { the normal arterial wall; } \\
\text { existence of appropriate } \\
\text { equipment; } \\
\text { risks of complications as } \\
\text { bleeding or paralysis. }\end{array}$ \\
\hline & Big & $\begin{array}{l}\text { mimics restenosis after } \\
\text { balloon angioplasty; } \\
\text { ease of handling; } \\
\text { use of devices for humans. }\end{array}$ & $\begin{array}{l}\text { denudation is made on } \\
\text { the normal arterial wall. }\end{array}$ \\
\hline Wire Injury & Small & $\begin{array}{l}\text { mimics restenosis after } \\
\text { balloon angioplasty; } \\
\text { ease of handling; } \\
\text { minimal mortality rate; } \\
\text { low costs related to animal } \\
\text { purchase and care; } \\
\text { availability of various } \\
\text { transgenic and knockout } \\
\text { strains; } \\
\text { no physical impairment. }\end{array}$ & $\begin{array}{l}\text { small size of the main } \\
\text { arteries; } \\
\text { requires less qualified } \\
\text { surgical expertise; } \\
\text { denudation is made on } \\
\text { the normal arterial wall; } \\
\text { existence of appropriate } \\
\text { equipment. }\end{array}$ \\
\hline
\end{tabular}




\begin{tabular}{|l|l|l|l|}
\hline Stent implantation & Small & $\begin{array}{l}\text { mimics restenosis and } \\
\text { thrombosis after stent } \\
\text { implantation; } \\
\text { low costs related to animal } \\
\text { purchase and care; } \\
\text { availability of various } \\
\text { transgenic and knockout } \\
\text { strains. }\end{array}$ & $\begin{array}{l}\text { small size of the main } \\
\text { arteries; } \\
\text { requires qualified surgical } \\
\text { expertise; } \\
\text { small stents not available; } \\
\text { denudation is made on } \\
\text { the normal arterial wall; } \\
\text { increased mortality; } \\
\text { existence of appropriate } \\
\text { equipment; } \\
\text { risks of complications as } \\
\text { bleeding or paralysis. }\end{array}$ \\
\cline { 2 - 3 } & Big & $\begin{array}{l}\text { mimics restenosis } \\
\text { and thrombosis after stent } \\
\text { implantation; } \\
\text { ease of handling; } \\
\text { use of devices for humans. }\end{array}$ & $\begin{array}{l}\text { denudation is made on } \\
\text { the normal arterial wall. }\end{array}$ \\
& & \\
\hline
\end{tabular}

\section{Disclosures}

There are no disclosures by the authors.

\section{Acknowledgement}

This work was supported by Interdisciplinary Centre for Clinical Research IZKF Aachen (junior research group to E.A.L.) within the faculty of Medicine at RWTH Aachen University. We also thank Mrs. Roya Soltan for help with the immunohistochemistry staining. 


\section{References}

1. Simsekyilmaz S, Liehn EA, Militaru C, Vogt F. Progress in interventional cardiology: challenges for the future. Thrombosis and haemostasis. 2015;113(3):464-472.

2. Kubo N, McCurdy S, Boisvert WA. Defective Fas Expression on Bone Marrow Derived Cells Alters Atherosclerotic Plaque Morphology in Hyperlipidemic Mice. Discoveries. 2015;3:e37.

3. Saffarzadeh M, Cabrera-Fuentes HA, Veit F, et al. Characterization of rapid neutrophil extracellular trap formation and its cooperation with phagocytosis in human neutrophils. Discoveries. 2014;2(2):e19.

4. Lindner V, Fingerle J, Reidy MA. Mouse model of arterial injury. Circ Res. 1993;73(5):792-796.

5. Schwartz RS, Edelman ER, Carter A, et al. Preclinical evaluation of drugeluting stents for peripheral applications: recommendations from an expert consensus group. Circulation. 2004;110(16):2498-2505.

6. Schwartz RS, Huber KC, Murphy JG, et al. Restenosis and the proportional neointimal response to coronary artery injury: results in a porcine model. $\mathrm{J}$ Am Coll Cardiol. 1992;19(2):267-274.

7. Curaj A, Wu Z, Fokong $S$, et al. Noninvasive molecular ultrasound monitoring of vessel healing after intravascular surgical procedures in a preclinical setup. Arterioscler Thromb Vasc Biol. 2015;35(6):1366-1373.

8. Liehn EA, Schober A, Weber C. Blockade of keratinocyte-derived chemokine inhibits endothelial recovery and enhances plaque formation after arterial injury in ApoE-deficient mice. Arterioscler Thromb Vasc Biol. 2004;24(10):1891-1896.

9. Liehn EA, Zernecke A, Postea O, Weber C. Chemokines: inflammatory mediators of atherosclerosis. Archives of physiology and biochemistry. 2006;112(4-5):229-238.

10. Simsekyilmaz S, Cabrera-Fuentes HA, Meiler S, et al. Role of extracellular RNA in atherosclerotic plaque formation in mice. Circulation. 2014;129(5):598-606. 
11. Wu Z, Curaj A, Fokong $\mathrm{S}$, et al. Rhodamine-loaded intercellular adhesion molecule-1-targeted microbubbles for dual-modality imaging under controlled shear stresses. Circ Cardiovasc Imaging. 2013;6(6):974-981.

12. Schober A, Zernecke A, Liehn EA, et al. Crucial role of the CCL2/CCR2 axis in neointimal hyperplasia after arterial injury in hyperlipidemic mice involves early monocyte recruitment and CCL2 presentation on platelets. Circ Res. 2004;95(11):1125-1133.

13. Ali ZA, Alp NJ, Lupton $\mathrm{H}$, et al. Increased in-stent stenosis in ApoE knockout mice: insights from a novel mouse model of balloon angioplasty and stenting. Arterioscler Thromb Vasc Biol. 2007;27(4):833-840.

14. Chamberlain J, Wheatcroft M, Arnold N, et al. A novel mouse model of in situ stenting. Cardiovasc Res. 2010;85(1):38-44.

15. Rodriguez-Menocal L, Wei Y, Pham SM, et al. A novel mouse model of instent restenosis. Atherosclerosis. 2010;209(2):359-366.

16. Simsekyilmaz S, Schreiber F, Weinandy S, Gremse F, Sonmez TT, Liehn EA. A murine model of stent implantation in the carotid artery for the study of restenosis. J Vis Exp. 2013(75):e50233.

17. Schroder K, Kohnen A, Aicher A, et al. NADPH oxidase Nox2 is required for hypoxia-induced mobilization of endothelial progenitor cells. Circ Res. 2009;105(6):537-544. 



\section{Chapter 4}

\section{Noninvasive molecular ultrasound}

monitoring of vessel healing following intravascular surgical procedures in a preclinical setup

Adelina Curaj, Zhuojun Wu, Stanley Fokong, Elisa A. Liehn, Christian Weber, Alexandrina Burlacu, Twan Lammers, Marc van Zandvoort, Fabian Kiessling Arterioscler Thromb Vasc Biol. 2015 Jun;35(6):1366-73. doi: 10.1161/ATVBAHA.114.304857.

PMID: 25838431 


\section{Abstract}

Objective: Cardiovascular interventions induce damage to the vessel wall making antithrombotic therapy inevitable until complete endothelial recovery. Without a method to accurately determine the endothelial status, many patients undergo prolonged anticoagulation therapy, denying them any invasive medical procedures such as surgical operations and dental interventions. Therefore, we aim to introduce molecular ultrasound imaging of the vascular cell adhesion molecule-1 (VCAM-1) using targeted poly-n-butylcyanoacrylate microbubbles (MBVCAM-1) as an easy accessible method to monitor accurately the reendothelialization of vessels.

Approach and Results: $A p o E^{-/}$mice were fed with an atherogenic diet for 1 and 12 weeks and subsequently, endothelial denudation was performed in the carotid arteries using a guide-wire. Molecular ultrasound imaging was performed at different time points after denudation (1, 3, 7, 14d). An increased MBvcAM-1binding after $1 d$, a peak after $3 d$ and a decrease after $7 d$ was found. After 12 weeks of diet MBVCAM-1 binding also peaked after 3d but remained high until $7 \mathrm{~d}$, indicating a delay in endothelial recovery. Two-photon laser scanning microscopy (TPLSM) imaging of double fluorescence staining confirmed the exposure of VCAM-1 on the superficial layer after arterial injury only during the healing phase. After complete reendothelialization, VCAM-1 expression persisted in the subendothelial layer but was not reachable for the MBVCAM-1 anymore.

Conclusion: Molecular ultrasound imaging with MBvCAM-1 is promising to assess vascular damage and to monitor endothelial recovery after arterial interventions. Thus, it may become an important diagnostic tool supporting the development of adequate therapeutic strategies to personalize anticoagulant and antiinflammatory therapy after cardiovascular intervention. 


\section{Introduction}

Restenosis and in-stent thrombosis following revascularization procedures are major obstacles in the local treatment of atherosclerotic plaques ${ }^{1,2}$. It has been shown that an intact endothelial layer has antithrombotic and atheroprotective properties $^{3}$, acting as a barrier against lipid uptake and inflammatory cell recruitment ${ }^{4}$. Even though drug-eluting stents reduce restenosis through the suppression of vascular smooth muscle cell (SMC) proliferation and migration ${ }^{5,6}$, complications such as in-stent thrombosis are still notable events ${ }^{7,8}$ due to delayed endothelial recovery.

The regrowth of the endothelium downregulates intimal smooth muscle cell proliferation, retards plaque development ${ }^{4,9}$, and consequently reduces the risk for post-interventional complications. Therefore, the monitoring of vascular restoration is important in therapeutic post-interventional follow-up. Suitable markers for monitoring vascular damage should ideally be expressed immediately after arterial denudation, and should remain to be expressed until reendothelialization is complete. Consequently, such markers have to be expressed on both, the vascular SMC (being exposed to the blood after endothelial removal) and the activated, regenerating endothelial cells (EC), which start covering the vascular wound (Supplemental Figure I). Finally, the markers should show a low luminal exposure under physiological conditions.

Ultrasound (US) imaging using targeted microbubbles (MB) can potentially provide valuable insights into the presence of prominent inflammation surface markers during the various states of endothelial regeneration, allowing the personalization of anti-inflammatory therapy in dosage, medication period and combination.

In the present study, we propose vascular cell adhesion molecule -1 (VCAM-1) as an US imaging biomarker of endothelial healing in big vessels such as carotid arteries. VCAM-1 is expressed by activated vascular SMC and it is accessible for intravascular targeting after arterial denudation, while replicating EC express 
VCAM-1 during endothelial reconstruction until regeneration ${ }^{10,11}$ (Supplemental Figure I). We therefore used VCAM-1-targeted MB (MBVCAM-1) and molecular US to monitor arterial endothelial recovery, and validated the results employing TPLSM as a deep-tissue imaging modality. TPLSM was applied for the characterization of VCAM-1 expression, MB binding, and morphological changes in the arterial lumen on cellular and subcellular levels. Our data provide strong evidence that MBVCAM-1 are suitable for noninvasive molecular US monitoring of vascular healing in a murine model of endothelial denudation.

\section{Methods}

\section{Synthesis of poly $n$-butylcyanoacrylate target-specific MB}

Rhodamine loaded poly-n-butylcyanoacrylate (PBCA)-stabilized, air-filled MB were prepared and functionalized as previously described ${ }^{12,13} .1 \times 10^{7}$ streptavidincoated MB in HEPES/Triton buffer ( $\mathrm{pH} 7.0)$ were mixed with $5 \mu \mathrm{g}$ of biotinylated anti-VCAM-1 antibody (clone 429, eBioscience) and incubated at room temperature for 30 minutes. Conjugated $M B$ were separated from excess antibody by 30 minutes flotation and re-suspended in $50 \mu \mathrm{L}$ HEPES/Triton buffer.

\section{Atherogenic murine model of restenosis}

Animal experiments were approved by local authorities and complied with the European Convention of Animal Protection law. Two similar animal models of accelerated atherosclerosis were used in this study: 1) eight-week old $\mathrm{ApoE}^{-/-}$ mice were fed an atherogenic diet (Altromin, Germany) for 1 week before and 2 weeks after the carotid injury (named short-term diet group); 2) mice were fed an atherogenic diet for 12 weeks before and 2 weeks after the arterial denudation (named long-term diet group). The arterial injury was performed using a coated guide wire as previously described ${ }^{14}$. A total number of 122 animals were used for this study, consisting of $n=29$ for ex vivo TPLSM studies, $n=15$ for immunohistology, $n=12$ for ex vivo US, $n=25$ for in vivo TPLSM and $n=41$ for in 
vivo US. Following the wire-injury procedure, VCAM-1 expression was measured at different time points $(1,3,7,14$ days post-injury).

\section{Operative procedures for flow-chamber experiments}

For each time point (1, 3, 7, 14 d) after arterial injury, mice were killed by intraperitoneal (i.p.) overdose injection of $400 \mathrm{mg} / \mathrm{ml}$ ketamine and $40 \mathrm{mg} / \mathrm{ml}$ xylazine. In addition, a terminal blood collection was performed via intracardiac puncture. The common carotid arteries $(6-7 \mathrm{~mm})$ were excised by restricted handling of both ends of the blood vessel to exclude luminal alterations. In order to avoid air bubbles within the lumen and tissue dryness, the blood vessels were preserved at $4^{\circ} \mathrm{C}$ in $1 \mathrm{x}$ Hanks Balanced Salt Solution (HBSS, pH 7.4, Life Technologies) during ex vivo imaging preparations. Each excised carotid artery was mounted in a customized flow chamber and then filled with HBSS $\left(37^{\circ} \mathrm{C}\right)$ as previously described ${ }^{15}$. For the US flow chamber experiments, we used a $7 \mathrm{~cm}$ deep flow-chamber to ensure US-waves propagation and the carotid artery was brought into the field of view by immersing the scan-head into the liquid. To mimic physiological conditions inside the mounted carotid arteries, fresh blood collected from healthy volunteers was anti-coagulated with heparin $(10 \mathrm{U} / \mathrm{ml})$ - EDTA (1.2 - $2 \mathrm{mg}$ EDTA $/ \mathrm{ml}$ ) and infused through the carotids preserving the physiological flow direction. Subsequently, MB suspended in whole blood, were infused for 10 minutes, followed by a 4 minutes washing step using human blood and a second washing step using HBSS.

\section{Ex vivo whole mount artery TPLSM imaging of wire-injured carotids}

Animals $(n=20)$ were operated as described above. At 1, 3, 7 and 14 d postsurgery, mice ( $n=5$, for each time point) were sacrificed via overdose i.p. injection of anesthetics. After mounting and flushing the carotids, cell nuclei (SYTO 41), endothelial PECAM-1 (CD31), and VCAM-1 (CD106) were stained by an onestep incubation. $400 \mu$ of HBSS containing anti-CD31 Oregon Green 488 (2.5 $\mu \mathrm{g} / \mathrm{mL})$, anti-CD106 Alexa Fluor $568(2.5 \mu \mathrm{g} / \mathrm{mL})$, and SYTO 41 (5 nM) were used 
to flush the carotid artery. Incubation was performed at transluminal pressure of $80 \mathrm{mmHg}$ for 1 hour. After flushing the artery with HBSS, imaging was performed using an Olympus FV1000MPE multiphoton microscopy system (Mai Tai DeepSee pulsed Ti:Sapphire laser with 140 fs pulse width at an excitation wavelength of $800 \mathrm{~nm}$ ). A 25x water dipping objective with numeric aperture (NA) of 1.05, working distance (WD) of $2 \mathrm{~mm}$, and with optical zoom capability was used. Three internal photon multiplier tubes were used to detect the fluorescence signals and filters were adjusted to the corresponding spectra: 418-468 $\mathrm{nm}$ for SYTO 41, 495-540 nm for elastin autofluorescence and CD31, and 595-650 nm for CD106. TPLSM images were analyzed using Image-Pro Analyzer 7.0 software (Media Cybernetis, Inc.) and Imaris software (Bitplane).

\section{Flow chamber experiments on the binding of MBVCAM-1 to carotid arteries after wire-injury using molecular US}

High-resolution imaging of the excised carotid arteries $1 \mathrm{~d}$ after the arterial denudation $(n=12)$ was performed with a linear high-frequency transducer (55 $\mathrm{MHz}$, mechanical index 0.9, peak negative pressure 6.7 MPa, axial resolution 30 $\mu \mathrm{m}$, lateral resolution $70 \mu \mathrm{m}$, focal length $4.5 \mathrm{~mm}$, depth of field $1.4 \mathrm{~mm}$, field of view $10.9 \mathrm{~mm}$ ) connected to a small animal US imaging system (Vevo 770, VisualSonics Inc) using a long-axis imaging plane. Measurements were performed using the contrast mode, which makes use of the enhanced scattering of MB at low US energies (4\% power, mechanical index 0.04 ) to distinguish them from tissue response. Ten minutes after MB infusion, a visible steady-state confirmed a homogeneous MB distribution in the circulating blood before the destructive pulse (Figure 3). After 10 minutes of continuous flow, the MBs-blood mixture was removed from the circuit by two washing steps (first with blood and then with HBSS as mentioned above). For competitive binding experiments, the carotid lumen was incubated with a 20 -fold higher concentration of free antiCD106 antibody $(100 \mu \mathrm{g}, 2 \mu \mathrm{g} / \mu \mathrm{l})$ for $1 \mathrm{~h}$ prior to MB injection. To quantify the amount of bound $\mathrm{MB}$ in the imaged area, an imaging sequence of 60 seconds (10 frames/second) was acquired. To prevent MB destruction, the first third of the sequence recorded images at a low transducer output power of $4 \%$. 
Subsequently, a destructive pulse (100\% power) destroyed all MB in the imaged area. The remaining imaging sequence was acquired at $4 \%$ power. Image analysis was performed by subtracting the mean value of grayscale intensity for several post-destructive frames (HBSS only) from the mean value of several predestructive frames (bound $\mathrm{MB}$ ). The resulting grayscale intensity difference represents the amount of signal generated by stationary MB.

\section{Flow chamber experiments on the binding of $\mathrm{MB}_{\mathrm{VCAM}-1}$ to carotid arteries after wire-injury using TPLSM}

Three days post wire-injury, animals $(n=9)$ were sacrificed and the carotid arteries were mounted as described above. Prior to the binding step, the arteries were stained with OG488 labelled endothelial PECAM-1 (CD31) and SYTO 41. For competitive binding experiments, the carotid lumen was incubated with a $20 x$ higher free anti-CD106 antibody concentration for $1 \mathrm{~h}$ prior to MB injection. After the infusion of $\mathrm{MB}$ and washing, imaging was performed using the Olympus FV1000MPE multiphoton microscopy systems. PECAM, SYTO 41 and CD106 filter settings were selected as described above. Rhodamine-labeled MB were imaged using an excitation wavelength of $840 \mathrm{~nm}$ and a spectral filter for the ranging 595-650 $\mathrm{nm}$.

\section{In vivo molecular US imaging}

Mice with wire-injured carotid arteries $(n=41)$ were investigated by molecular US imaging at different time points (1, 3, 7 and $14 \mathrm{~d})$. For catheterization and imaging the mice were anaesthetized with $1 \%$ isoflurane and placed on a heated table (Vevo Mouse Handling Table). In vivo molecular US measurements were performed using a small animal high frequency US system (Vevo 770, VisualSonics) and a $40 \mathrm{MHz}$ transducer (mechanical index 1.2, peak negative pressure $6.6 \mathrm{MPa}$, axial resolution $40 \mu \mathrm{m}$, lateral resolution $80 \mu \mathrm{m}$, focal length 6 $\mathrm{mm}$, depth of field $1.5 \mathrm{~mm}$, field of view $14.5 \mathrm{~mm}$ ). MB-specific signals were acquired and color-coded overlays on B-mode images were performed using the contrast mode provided by the vendor. A dose of $2 \times 10^{7}$ MBVCAM-1 or MB control 
(MB $\mathrm{MTR}_{\mathrm{C}}$ ) was injected intravenously via the cannulated tail vein under US control (4\% transmitted power, mechanical index 0.036). For competitive binding studies at $1 \mathrm{~d}$ after wire-injury, intravenous injection of 20-fold higher concentration of free anti-CD106 antibody (100 $\mu \mathrm{g}, 2 \mu \mathrm{g} / \mu \mathrm{l})$ was applied to each mouse 10 minutes before MBVcAM-1 injection to block VCAM-1-binding sites. In all experiments successful MB injection was confirmed by assessing an imaging sequence of 60 seconds (10 frames/second) during the injection. Seven minutes after MB injection, molecular US imaging in contrast mode was performed as described above to measure the difference in the signal levels before and directly after the destructive pulse ${ }^{16}$.

\section{In vivo TPLSM}

For in vivo measurements, wire-injured $\mathrm{ApoE}^{-/-}$mice $(n=25)$ were anesthetized using an i.p. injection of ketamine $(100 \mathrm{mg} / \mathrm{kg})$ and medetomidine $(10 \mathrm{mg} / \mathrm{kg})$. Body temperature was kept at $37^{\circ} \mathrm{C}$ by means of a heating pad. Then, the carotid artery was carefully exposed. For MB injections, a catheter made of a $0.28 \mathrm{~mm}$ polyethylene tubing adapted to a $30 \mathrm{G}$ needle was inserted into the tail vein and flushed with $\mathrm{NaCl}$-heparin solution. Thirty minutes before imaging $0.5 \mathrm{nM}$ SYTO 13 was applied superficially on the top of the exposed carotid in order to stain the vessel adventitia for orientation within the tissue.

TPLSM imaging was performed using a Leica SP5 (Leica Microsystems, Germany) with an integrated resonance scanner for high-speed imaging and a Compact Ultrafast Ti:Sapphire Laser (Chameleon, Coherent, USA) at an excitation wavelength of $800 \mathrm{~nm}$ and a 20x water dipping objective (NA=1.0, $\mathrm{WD}=2 \mathrm{~mm}$, with optical zoom capability). Two internal photon multiplier tubes were used to detect the fluorescence signals and filters were adjusted to the corresponding spectra: 505- $560 \mathrm{~nm}$ for SYTO 13, 590-650 nm for rhodamine. In vivo imaging was performed at a frame size of $400 \times 400$ pixels and with a scan rate of $8000 \mathrm{~Hz}$. Line averaging of 2 was applied for noise reduction, resulting in a final imaging speed of 10 frames per second. For time lapse recording, a series of 500 images or 55 second of video material was recorded. After the injection of 


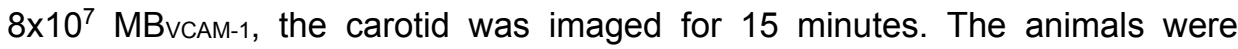
sacrificed after in vivo imaging and additional ex vivo carotid imaging at higher resolution was performed, as described above.

\section{Immunohistology of denudated arteries from long-term diet group}

Mice $(n=15)$ fed for 12 weeks with an atherogenic diet (HFD) were subjected to arterial denudation and then sacrificed at different time point $(1,3,7,14$ and 21 d). For immunhistology, perfusion fixation with $4 \%$ paraformaldehyde (P-6148, Sigma) and paraffin embedding was performed. Serial tissue sections $(5 \mu \mathrm{m})$ were generated starting at the bifurcation. Samples were stained for VCAM-1 (11444-1-AP) and CD31 (sc-1506) expression. FITC-, respectively Cy5 conjugated secondary antibodies were used for visualization, and sections were analyzed using the „Diskus” software (Hilgers).

\section{Statistical analysis}

Differences between groups of ex vivo and in vivo $\mathrm{MB}$ adhesion assays were analyzed using the two-tailed Student's t-test, considering $p<0.05$ to display significant differences. To correct for multiple group comparisons, we performed Bonferroni correction. Error bars shown on graphs represent the standard deviation. Statistical analysis was performed using GraphPad Prism 5.0 (GraphPad Software).

\section{Results}

\section{Ex vivo whole mount artery TPLSM imaging of wire-injured carotids}

Prior to endothelial denudation, animals were fed a high cholesterol diet for either 1 week (short-term diet) or 12 weeks (long-term diet). The majority of experiments were conducted on animals with a short-term diet to induce hyperlipidemia, in 


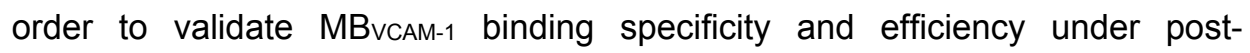
interventional conditions. The long-term diet group was introduced to investigate the impact of high cholesterol diet on the rate of endothelial regeneration after plaque removal by wire-injury and the potential of molecular US to distinguish between both conditions.

First, the VCAM-1 expression pattern and the degree of endothelial regeneration were studied in mice on short-term diet after endothelial denudation. We stained ex vivo mounted carotid arteries for PECAM-1, VCAM-1 and nuclei, and analysed marker expression using TPLSM at the four different time points $(1,3,7,14 \mathrm{~d}$ : Figures 1 and 2A). Three-dimensional reconstruction of the vessel wall and quantification of the endothelial regeneration showed only endothelial cell debris at $1 \mathrm{~d}$, and an endothelial coverage of $18 \%$ after $3 \mathrm{~d}, 80 \%$ after $7 \mathrm{~d}$, and $94 \%$ after $14 \mathrm{~d}$, respectively (Figure $2 \mathrm{~B}$ ).

The endothelial cell debris at $1 \mathrm{~d}$ was positive for VCAM-1 (Figure 1). At this time point, VCAM-1 was found to be highly expressed on vascular SMC as well. The first signs of endothelial regeneration were visible 3 days after denudation, in conjunction with the highest colocalization of VCAM-1 with the endothelial marker PECAM-1, indicating pronounced EC activation and regeneration (Figure $2 \mathrm{C}$ ). In parallel, the vascular SMC exposed to the vascular lumen also displayed a strong VCAM-1 expression. At later time points ( $7 \mathrm{~d}$ and $14 \mathrm{~d})$, EC regeneration progressed further, showing a continuous decrease in VCAM-1 expression down to the level of noninjured controls after $14 \mathrm{~d}$ (Figure 1, Figure 2A).

In contrast, VCAM-1 expression on vascular SMC remained high even $14 \mathrm{~d}$ after denudation, however these vascular SMC were not luminally exposed anymore and thus not accessible to MBvcam-1. These findings are in line with the mean fluorescence intensity quantifications for VCAM-1 and PECAM-1 expression (Supplemental Figure II). 

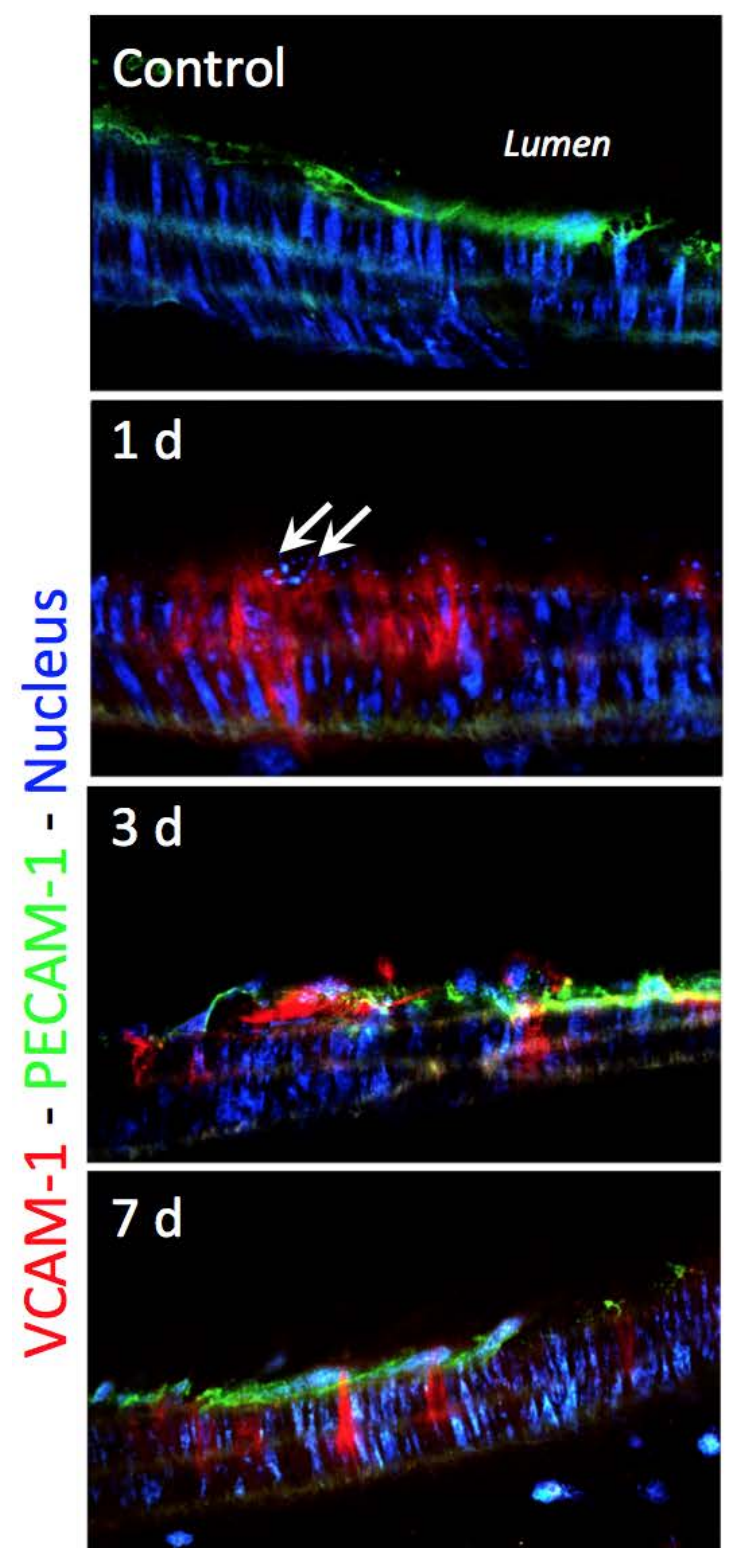

$14 \mathrm{~d}$

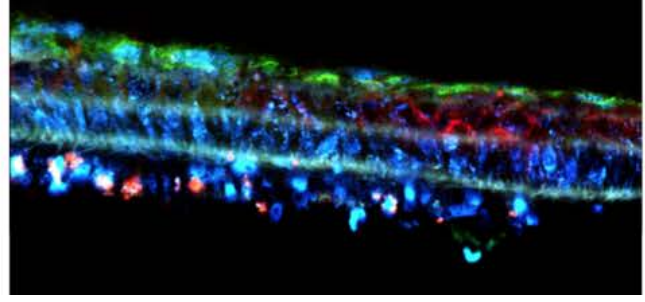

Figure 1. Ex vivo whole mount staining and TPLSM imaging of denudated carotid arteries of mice from short-term atherogenic diet group. For the staging of endothelial regeneration and VCAM-1 expression, mice were sacrificed at timepoint 1,3,7 and $14 \mathrm{~d}$ post denudation and the vital tissue was mounted and stained. One day post surgery, the endothelium (green = PECAM-1) is absent, showing only EC debris in the lumen (indicated by white arrows). The VCAM-1expressing vascular SMC are exposed to the blood flow (red = VCAM-1). Three days post denudation, at the onset of endothelial regeneration, there is strong expression of VCAM-1 by both vascular SMC and EC (blue = SYTO 41). Seven days post denudation, there is a partial reconstruction of the endothelial layer covering VCAM-1-positive vascular SMC. A complete endothelial recovery is found after $14 \mathrm{~d}$. Images were processed using Image Pro 7.0. Scale bar $=50 \mu \mathrm{m}$. 

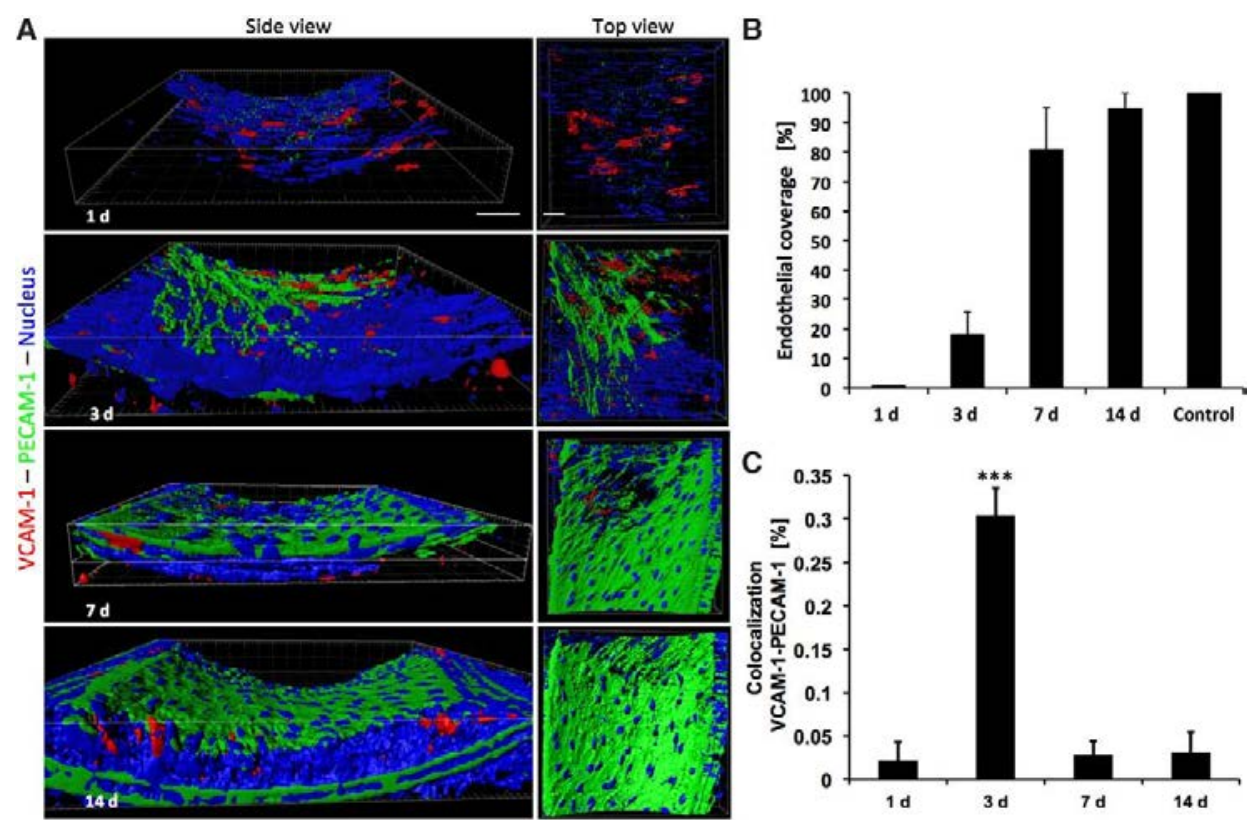

Figure 2. Three-dimensional rendering of denudated carotid arteries of mice from the shortterm atherogenic diet group and quantification of endothelial recovery. Using PECAM-1 as a marker for endothelial cells and VCAM-1 for endothelial regeneration, z-stack images were rendered to better illustrate the extent of regeneration and inflammation. A) The degree of endothelial regeneration is depicted starting from $1 \mathrm{~d}$ until $14 \mathrm{~d}$ post denudation. B) Graph reporting the percentage of endothelial coverage in carotid arteries before and after denudation, compared to control vessels. While directly after denudation, almost the entire vessel surface was removed, $94 \%$ of the endothelial surface coverage was reconstituted after $14 \mathrm{~d}$. C) To illustrate the endothelial availability of VCAM-1, the Manders coefficient for VCAM-1 colocalization with PECAM-1 for all time points was calculated. Scale bar $=25 \mu \mathrm{m}$.

\section{US analysis of MB VCAM-1 $_{1}$ binding to carotid arteries after wire- injury in a flow chamber}

MBVCAM-1 binding to carotid arteries was studied in mice from the short-term diet group, $1 \mathrm{~d}$ after endothelial denudation. First, the efficiency and specificity of MBVCAM-1 binding to VCAM-1 under flow conditions in early-denudated carotid arteries was investigated ex vivo. A 9-fold higher signal decrease after MBvcam1 destruction in denudated carotid arteries compared to the control carotid arteries was found $(p<0.001)$. In addition, a $~ 8$-fold higher signal decrease after MB 
destruction was obtained in denudated carotid arteries when compared to the competitive binding group as well as to untargeted $M B(p<0.05$ and $p<0.05$ respectively) indicating strong binding of MBVCAM-1 to its target (Figure 3).
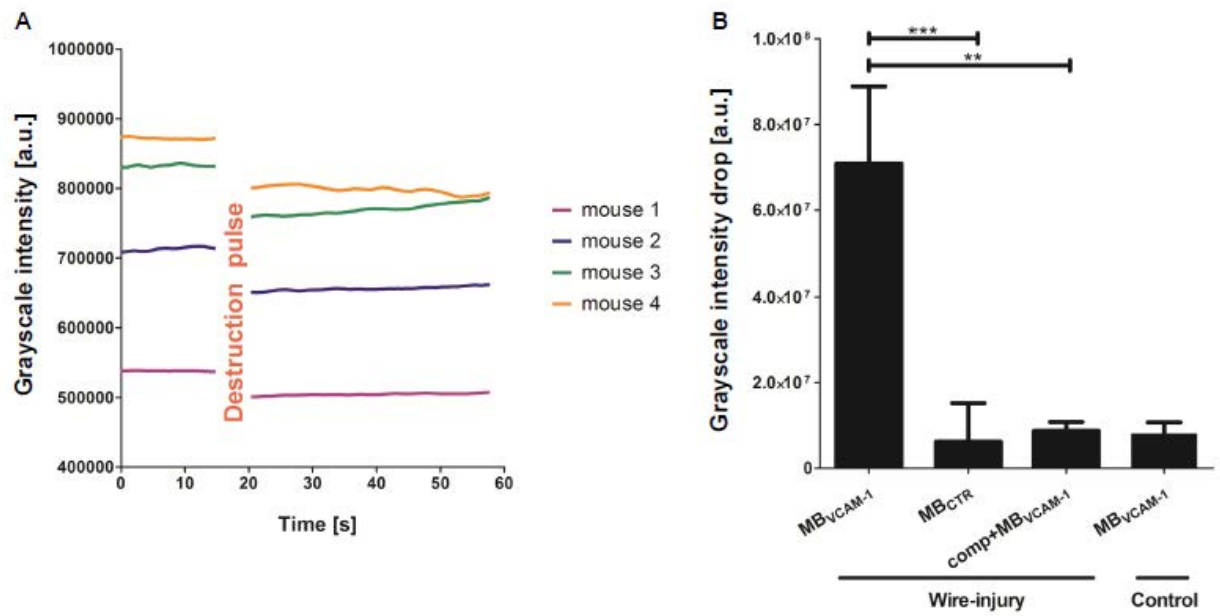

Figure 3. Ex vivo molecular US imaging of denudated carotid arteries of mice from short-term atherogenic diet group. To investigate the specificity of MB VCAM-1 $_{1}$ binding to VCAM-1 in earlydenudated carotid arteries and to evaluate the potential in vivo application of the imaging method, ex

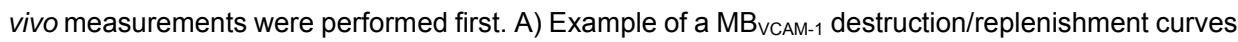
$(n=4)$ recorded in flow chamber experiments. Common carotid arteries were excised and mounted in a customized flow chamber. At time point 0 , the mixture of $\mathrm{MB}_{\mathrm{VCAM}-1}$ and blood which had been circulating for 10 mintes was removed and replaced with HBSS for imaging. B) Quantification and statistical analysis of MB binding ( $n=4$ mice per group; total of 12 animals) showing a significantly higher binding of $\mathrm{MB}_{\mathrm{VCAM}-1}$ to $1 \mathrm{~d}$ denudated carotid arteries compared to control carotids, as well as a significantly higher binding of $\mathrm{MB}_{\mathrm{VCAM}-1}$ to $1 \mathrm{~d}$ denudated carotid arteries compared to both, $\mathrm{MB}_{\mathrm{CTR}}$ and to the competitive binding group where carotid arteries were incubated with 20 -fold higher concentration of free anti-CD106 antibody.

\section{TPLSM analysis of MBVCAM-1 binding specificity to carotid arteries after wire-injury in a flow chamber}

Next, the extent and specificity of rhodamine-labelled MBvcam-1 binding was validated in flow-chamber experiments using TPLSM (Figure 4A). Three days after endothelial denudation, carotid arteries of mice from the short-term diet group were excised and perfused under physiological flow rates $(0.5 \mathrm{ml} /$ minute) 
with MBVCAM-1. After MBs binding and washing, the carotid arteries were stained for VCAM-1, PECAM-1 and nuclei, showing bound MB to be co-localized with the VCAM-1 signal. MBVCAM-1 binding to the denudated artery was $\sim 7$ fold higher compared to the control artery. Under competitive binding conditions, MBVCAM-1 accumulation was suppressed by a factor of $\sim 9$ (Figure $4 \mathrm{C}$ ).
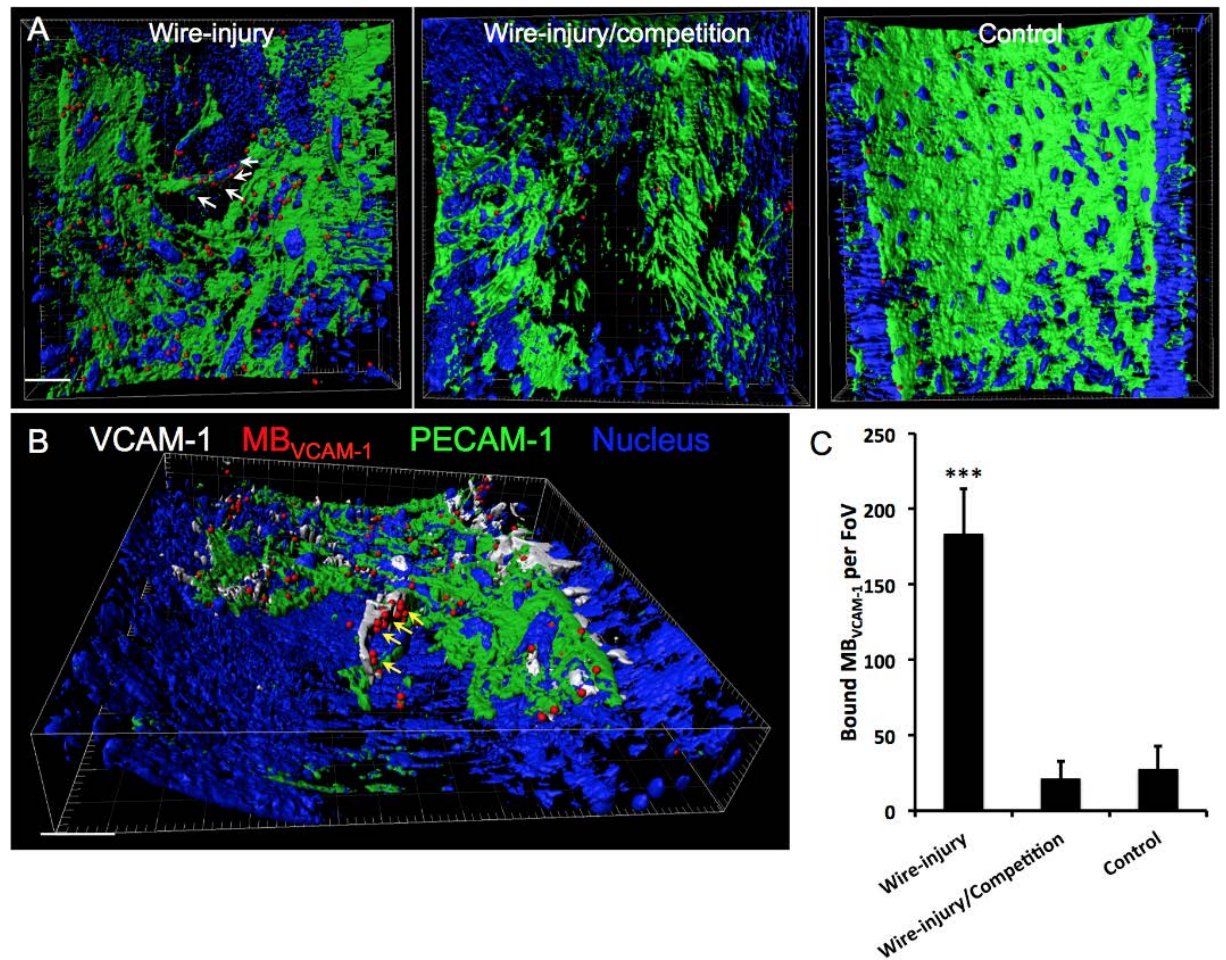

Figure 4. Ex vivo TPLSM flow chamber images of carotid arteries $3 \mathrm{~d}$ post endothelial denudation and of control arteries of mice from short-term atherogenic diet group. For validation of VCAM-1 as a molecular target under flow, MB VCAM-1 $_{\text {was }}$ flowed on denudated and control vessels. A) Rhodamine-labeled MB $_{V \text { CAM-1 }}$ strongly bind in denudated carotid arteries (white arrows) but hardly in control ones, as well as denudated ones upon competitive binding with excess free VCAM-1 antibody. B) Co-staining of PECAM-1, VCAM-1 and nuclei after MB VCAM-1 $_{\text {1 }}$ perfusion of denudated carotid arteries shows colocalization of MB $_{V C A M-1}$ with VCAM-1 staining (yellow arrows). C) Graph showing the results of the quantitative analysis of $\mathrm{MB}_{\mathrm{VCAM}-1}$ binding. In comparison to experiments with $\mathrm{MB}_{\mathrm{VCAM}-1}$ only a significantly lower binding in the presence of competing antibodies was found in denudated and control arteries. Scale bar $=50 \mu \mathrm{m}$. 


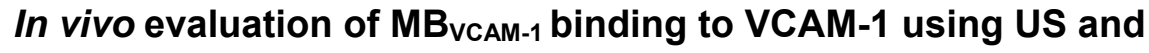 TPLSM}

Lastly, MBvcam-1 binding and detection was validated in vivo. First, in vivo MBVCAM- 1 binding was tested $1 \mathrm{~d}$ after endothelial denudation in mice from the short-term diet group. Using contrast mode, the MB influx after injection could be visualized (Figure 5A) and quantified (Figure 5B). After clearance of circulating $M_{\text {M }}{ }_{\text {CAM-1 }}$, bound MBVCAM-1 were found attached to the inner vessel wall (Figure $5 A)$.

Binding of significant amounts of MBVCAM-1 was further confirmed by a strong drop in US signal intensity after the application of a destruction pulse (Figure $5 \mathrm{C}$ ). Competitive binding experiments showed the specificity of MBVCAM-1 to its intended target at day 1 post-surgery in denudated carotid arteries: after blocking VCAM-1 with excess free antibody prior to MBVCAM-1 injection, there was a drop in MBVCAM-1-specific signals by $90( \pm 8) \%$ (Figure $5 \mathrm{D}$ ).

To verify MB binding, we performed in vivo TPLSM measurements with rhodamine-loaded ${ }^{13,15,17}$ MBvcAM-1 in wire-injured carotids. After creating a surgical window for TPLSM ${ }^{18}$, we tracked single-bound MB in a field of view of $500 \times 500 \mu \mathrm{m}$. Immediately after injection, we monitored the influx of circulating MB (Supplemental Figure IIIA), which was followed by a conducted in a separate group of animals (also $1 \mathrm{~d}$ after arterial injury), confirming specific MBVCAM-1 binding rapid clearance within approximately 5 minutes.

While untargeted and targeted MB under competitive binding conditions with free VCAM-1 antibodies did not significantly bind to the vascular wall of denudated carotid arteries ( $3 \mathrm{~d}$ after denudation), many stationary MBVCAM-1 were detected at the luminal side of the carotid artery for over more than 6 minutes (Supplemental Figure IIIB).

Even after euthanasia, excision and mounting of the carotid arteries, MBVCAM-1 were still present within the lumen. 
A
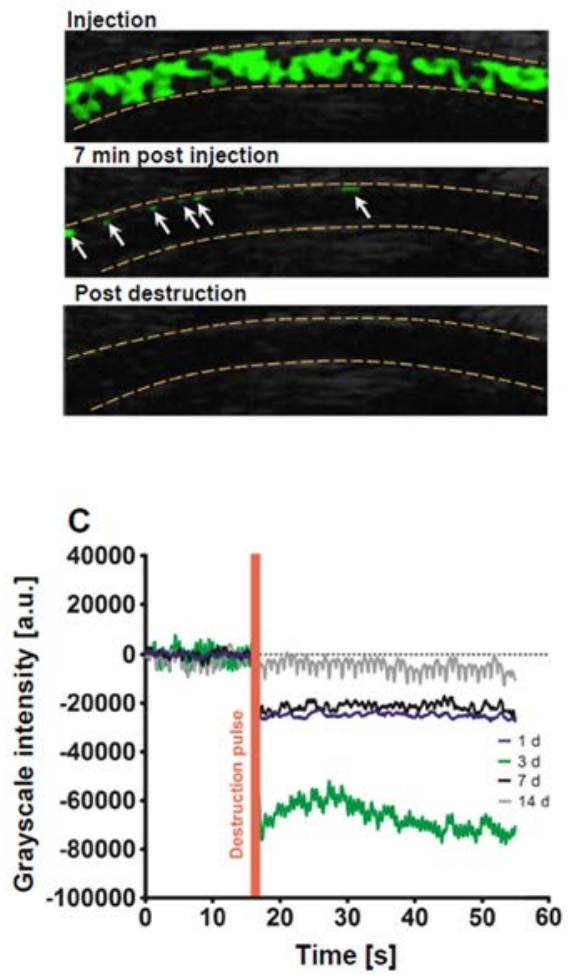

B

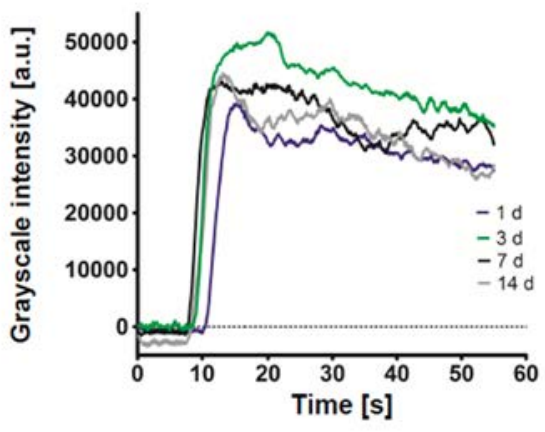

D

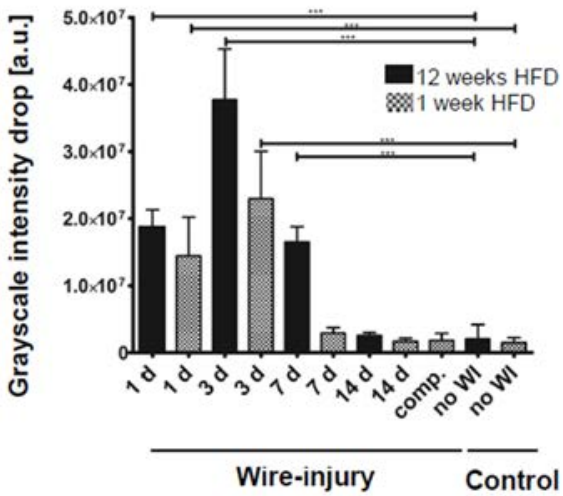

Figure 5. In vivo molecular US imaging of denudated carotid arteries. US monitoring of endothelial recovery in vivo was performed using the destruction-replenishment curve of injected $M_{\text {MCAM-1. }}$ Representative results from in vivo contrast mode molecular US imaging of murine carotid arteries in a long-axis imaging plane showing A) Upper panel - the contrast mode US image of circulating $\mathrm{MB}_{\mathrm{VCAM}-1}$ in the blood; middle panel - US image before the destructive pulse showing $M_{\text {VCAM- }}$ bound to their target; bottom-pannel - US image after $M_{\text {VCAM-1 }}$ destruction displaying a MBfree lumen. MB are color-coded in green; stationary MB are indicated with white arrows. B) The normalized in vivo $\mathrm{MB}$ injection curve shows an increase in grayscale intensity concomitantly with the inflow of the MB. C) Examples of normalized $\mathrm{MB}_{\mathrm{VCAM}-1}$ destruction-replenishment sequences for each time point after endothelial denudation (1, 3, 7 and $14 \mathrm{~d}$ ) in mice from long-term diet. Circulation time of $\mathrm{MB}_{\mathrm{VCAM}-1}$ before the destructive pulse was 7 minutes. $\left.\mathrm{D}\right)$ The quantification and statistical analysis of MB binding ( $n=4-5$ animals per group) indicates specific binding of $M_{V \text { VAM-1 }}$ to denudated carotid arteries (short-term high cholesterol diet - chess pattern bars, long-term high cholesterol diet - solid bars). Competitive-binding experiments were conducted in a separate group of animals (also 1 day after arterial injury), confirming specific $\mathrm{MB}_{\text {VCAM-1 }}$ binding. PECAM indicates platelet/endothelial cell adhesion molecule; and VCAM indicates vascular cell adhesion molecule. 


\section{Comparative US imaging using $\mathrm{MB}_{\mathrm{VCAM}-1}$ to assess endothelial} regeneration in mice on short- and long-term atherogenic diet in vivo

A short-term diet of one week before denudation only induces hyperlipidemia without plaque development. In contrary, a long-term diet (12 weeks) leads to signs of early plaque growth. Thus, it was of interest to confirm that our molecular US approach was capable to assess vascular regeneration in both cases and to compare the course of healing. A significantly enhanced binding of MBVCAM-1 in carotid arteries could only be observed at early time points after endothelial denudation, starting immediately ( $1 \mathrm{~d}$ ) and peaking at $3 \mathrm{~d}$ (Figure 5D) in both short- and long-term diet groups. In the short-term diet group quantification showed a $\sim 8$-fold increase in US grayscale intensity difference after $1 \mathrm{~d}$, and a $\sim 15$-fold increase after $3 d$, compared to the control group $(p<0.001$ and $p<0.001$ respectively), while at $7 \mathrm{~d}$ only a 2 -fold increase in US grayscale intensity difference compared to the control group $(p>0.05)$ was measured. At early time points similar results were obtained in the long-term diet group, presenting a 9fold increase in US grayscale intensity difference after $1 \mathrm{~d}$, and a 18-fold increase after $3 d,(p<0.05$ and $p<0.001$ respectively). However, after $7 d$ still a $\sim 8$-fold increase $(p<0.001)$ was found in the long-term diet group, indicating a delayed endothelial recovery after wire-injury, which translated into a prolonged VCAM-1 availability detected by US through MBVCAM-1 binding. Fourteen days post surgery, MBVCAM-1 binding dropped down to the level of unhandled control animals in both short- and long-term diet groups, showing that endothelial recovery can be monitored by US molecular imaging.

\section{Immunohistology of denudated arteries from long-term diet group}

Finally, we validated reendothelialisation and VCAM-1 expression in the carotid arteries of the long-term diet group by immunohistology at $1 \mathrm{~d}, 3 \mathrm{~d}, 7 \mathrm{~d}$, and $14 \mathrm{~d}$ after denudation (Supplemental Figure IV). In control carotid arteries VCAM-1 
was found in small spots at the endothelial surface (less than $3 \%$ of the total area), where native plaques were developing. One day after denudation, endothelial CD31 was absent, while VCAM-1 was present at the luminal side and in the media. Three and 7d after denudation, both CD31 and VCAM-1 were visible at the luminal side of the artery, while after $14 \mathrm{~d}$ VCAM-1 was only detected in the media or under the CD31-stained intima.

\section{Discussion}

The endothelial response to interventional procedures after blood flow restoration in atherosclerotic arteries is one of the most important factors determining patient recovery. The degree of endothelial damage correlates to the risk of neointima growth, platelet adhesion and thrombosis. Thus, it precludes the success of interventional surgery, i.e. slower endothelial regeneration causing a higher risk for complications ${ }^{7}$. After the intervention, dual anti-platelet medication and inflammatory inhibitors are applied using a standardized dosage, medication period, and combination, which is independent of the recovery rate of individual patients. However, the patient's age, general immune state, co-morbidities such as diabetes, and the different size of the plaque and injured area can strongly influence the speed of regeneration and degree of inflammation ${ }^{19,20}$. Thus, there appears to be a clear need for a reliable diagnostic assessment of endothelial regeneration, as well as for the individualization of post-interventional anticoagulant and anti-inflammatory therapy.

For this purpose, as a cheap and real-time imaging modality with excellent spatial resolution, molecularly targeted US imaging may be the modality of choice. However, the selection of the target for the molecular probe must be done carefully and the target should fulfil 3 criteria: 1) subendothelial expression after endothelial denudation (e.g. expression by luminally exposed vascular SMC); 2) expression by the activated regenerating EC; and 3) absence/low constitutive expression after reendothelialization. Previous studies have shown results indicating, that some markers may have a constitutive expression level (PECAM1, ICAM-1, P- and E-Selectin) ${ }^{11,15,21-23}$, other markers might either be not 
sufficiently expressed on activated vascular SMC (PECAM-1, E-Selectin, PSelectin $)^{14,21,24}$, or remain to be upregulated for a prolonged period of time after complete structural recovery (PECAM-1, ICAM-1) $)^{22}$ (Supplemental Table I and Supplemental Figure V).

Based on the current literature, VCAM-1 was chosen in this study as a target for several reasons: VCAM-1 is one of the most well-known atherosclerotic markers ${ }^{25}$. It has already been used for visualizing aortic atherosclerotic lesions by molecular US ${ }^{26-28}$. Despite its major application in atherosclerosis, VCAM-1 is also an acute inflammatory marker that is highly expressed after vascular injury, and it plays a major role in the formation of neointima and restenosis ${ }^{29}$. It was previously described that VCAM-1 is expressed by both $\mathrm{EC}^{30}$ and vascular SMC $^{31,32}$ in atherosclerotic arteries. However, binding of MBVCAM-1 to VCAM-1 is dependent on its luminal availability. Based on immunofluorescence quantifications and $3 \mathrm{D}$ renderings of the vessels, one day after endothelial denudation, activated medial vascular SMC expressing VCAM-1 are exposed to the blood flow, presenting the only source for MBVCAM-1 binding, since EC were removed by endothelial denudation. Three days after denudation, the endothelial layer is regenerating. The activated and proliferating ECs express VCAM-1 as well, presenting an additional source for VCAM-1. Consequently, MB binding peaks due to higher receptor availability. Subsequently, VCAM-1 expression by $E C$ decreases with endothelial regeneration. Therefore, significant colocalization of VCAM-1 with PECAM-1 could only be observed at $3 \mathrm{~d}$, due to the lack of EC after $1 \mathrm{~d}$, and the absence of endothelial activation after $14 \mathrm{~d}$, when endothelial coverage was close to complete. After endothelial recovery sub-endothelial and intimal markers, even though present and upregulated (Supplemental Figure I and Figure 1), are not accessible to MB anymore. The lack of MBVcaM-1 binding then indicates completion of the endothelial regeneration and the return of EC towards a physiological state. Mice fed for 14 weeks with atherogenic diet showed a delay in endothelial recovery. This is not surprising since high cholesterol diet and obesity related pathologies such as diabetes have been reported to interfere and delay wound healing and prolong the inflammatory phase, both in animals and patients ${ }^{33-35}$. Even though VCAM-1 was upregulated in the early plaque 
region of the control artery, significant MBVCAM-1 binding in mice with 12 weeks of diet was not detected. A possible explanation could be the limited size of the plaque area, comprising of only $3 \%$ of the total endothelial surface (data not shown), which may not be sufficient to induce visible MB binding above the background noise level.

Previously, US imaging was used for the noninvasive visualization of typical instent restenosis after carotid stenting of the internal carotid artery in humans ${ }^{36-38}$. In the present study, we used VCAM-1 not as a marker for targeted plaque imaging, but as an inflammation marker in the context of endothelial regeneration following intravascular procedures. The detection of delayed reendothelialization and prolonged endothelial inflammation induced by diet or drug eluting stents is an acute issue in clinical diagnostics. The results of our study open new perspectives to the application of molecular US for monitoring vascular healing in big vessels such as carotid arteries which are the main arteries subjected to revascularisation. Potential clinical translation was further encouraged by similar results collected in a pig model (own unpublished data).

Our study has limitations. Firstly, using MBvcAM-1, we can monitor endothelial regeneration and coverage, but this may not be equal to complete functional endothelial recovery. Thrombogenicity and complement activation may still be given even if VCAM-1 expression levels have been normalized. Therefore, other adhesion molecules involved in leukocyte recruitment and transendothelial migration, such as P-Selectin ${ }^{39,40}$, should be investigated in future studies as well, in order to provide evidence for a functional endothelial recovery. Secondly, streptavidin-coated MB are not clinically translatable, nor is the use of biotinylated antibodies. However, targeting peptides and antibodies can also be coupled using non-immunogenic linkers. This was recently demonstrated with clinically applicable PBCA MB based on a peptide ligand for specific imaging of E-Selectin expression in tumor blood vessels ${ }^{41}$, as well as with angiogenesis-specific VEGFR $2-M B$ in molecular imaging of breast and prostate cancer ${ }^{42,43}$, and with $\mathrm{P}$ and E-Selectin-targeted MB in inflammatory bowel disease ${ }^{44}$ and myocardial infarction ${ }^{45}$. Following these examples of clinically translatable molecular US 
contrast agents, the generation of a MBVCAM-1 with covalently attached targeting ligands would be a logical and practicable next step for future clinical implementation. Moreover, metal stents are not suitable for US imaging since the acoustic reflectivity of the stent would obstruct MB detection and impede healing assessments. Consequently, this diagnostic method can only be applyed in case of polymer-based stents and after endarterectomy.

In conclusion, we demonstrate that noninvasive molecular US monitoring of vascular healing after intravascular procedures is possible through luminal exposure of VCAM-1 when using VCAM-1-targeted MB as intravascular molecular probes. Therefore, molecular US imaging may become an important diagnostic tool supporting the development of adequate therapeutic strategies to reduce complications and to personalize anticoagulant and anti-inflammatory therapy after stent implantation or endarterectomy in carotid arteries for cerebral revascularisation.

\section{Acknowledgement}

This Research was supported by the RWTH-Aachen University IZKF core facility for Two-Photon Microscopy.

\section{Funding Sources}

This Research was supported by the Deutsche Forschungsgemeinschaft (DFG) Forschergruppe FOR809 and IZKF Aachen (junior research group to Dr. Liehn).

\section{Disclosures}

No relationship with industry exists. 


\section{Significance}

Thrombotic arterial occlusion due to endothelial damage is the main limitation of interventional blood flow restoration in atherosclerotic arteries. The current recommendation is preventive anticoagulant therapy for at least 12 months in case of drug-eluting stent implantation. However, anticoagulant therapy limits further surgical interventions and can also cause adverse effects like cerebral hemorrhage. Thus, it should be administered as short as necessary. Unfortunately, the optimal duration of antiplatelet therapy following various revascularization procedures remains unknown due to uncertainty of endothelial recovery.

Molecular ultrasound imaging is an exquisite tool for characterizing angiogenic and inflammatory vessels and initial clinical trials with targeted microbubbles show promising results. In this manuscript we provide preclinical evidence that VCAM-1 targeted microbubbles faithfully depict vessel damage and endothelial regeneration. Based on these findings, we hypothesize that molecular ultrasound imaging may become a powerful diagnostic tool to personalize antiplatelet therapy after vascular interventions, capable of improving the therapeutic outcome of patients. 


\section{Supplementary tables and figures}

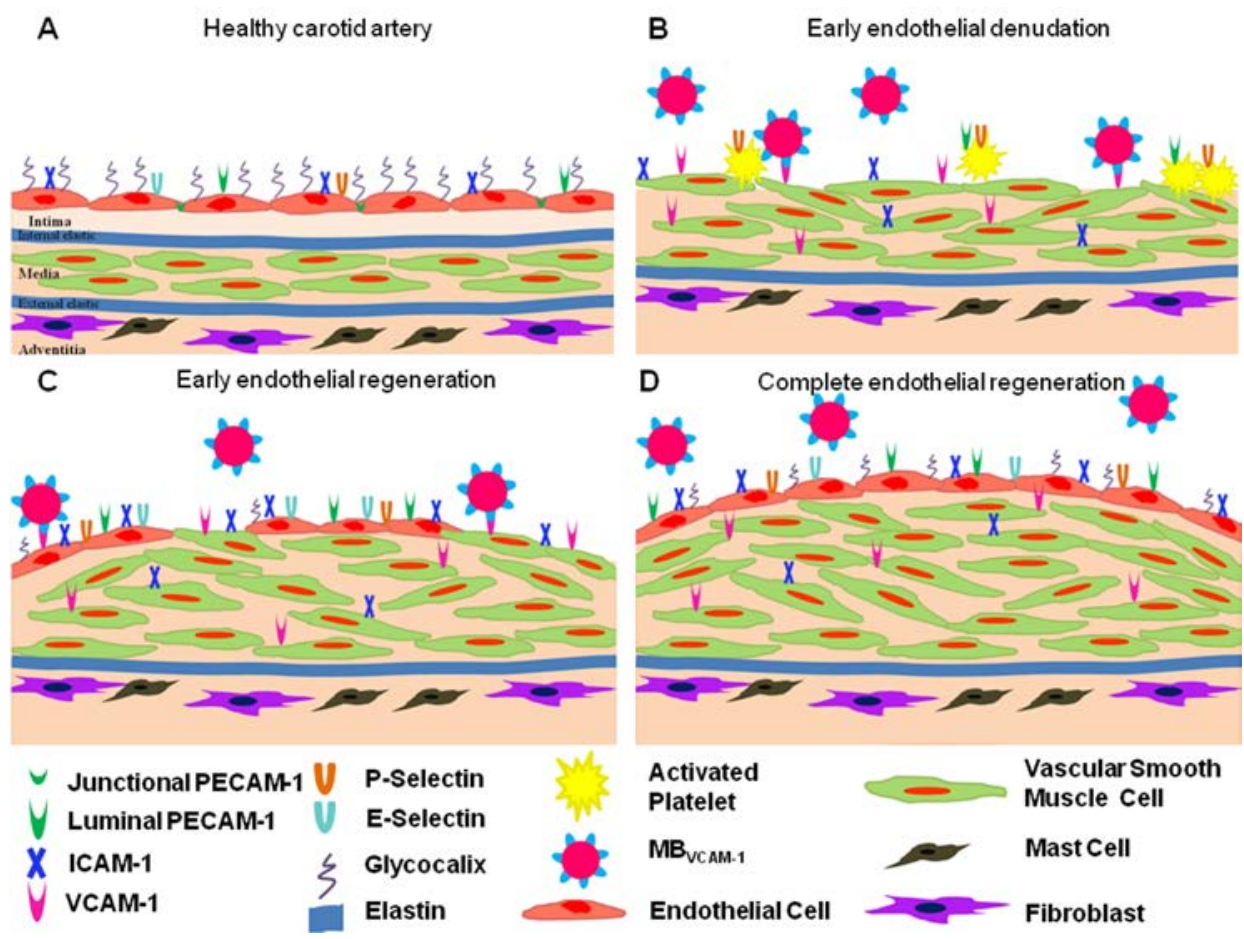

Supplemental Figure I. Scheme depicting the expression of luminal markers in healthy and wire-injured carotid arteries and its impact on $\mathrm{MB}_{\mathrm{VCAM}-1}-\mathrm{binding}$. A) endothelium constitutively expresses PECAM-1, ICAM-1, P- and E-Selectin. B) after endothelial denudation, vascular SMCrelated VCAM-1 and ICAM-1, as well as PECAM-1 and P-Selectin on attached thrombocytes are available on the luminal side of the artery. C) during endothelial recovery, PECAM-1, ICAM-1, VCAM1, P- and E-Selectin are upregulated on EC. Vascular SMC continue to express VCAM-1 and ICAM1. D) even after the endothelium is completely reconstructed, PECAM-1, ICAM-1, P- and E-Selectin will still be available on the luminal site for a prolonged period of time, while vascular SMC-related VCAM-1 and ICAM-1 become inaccessible for intravascular molecular targeting. 


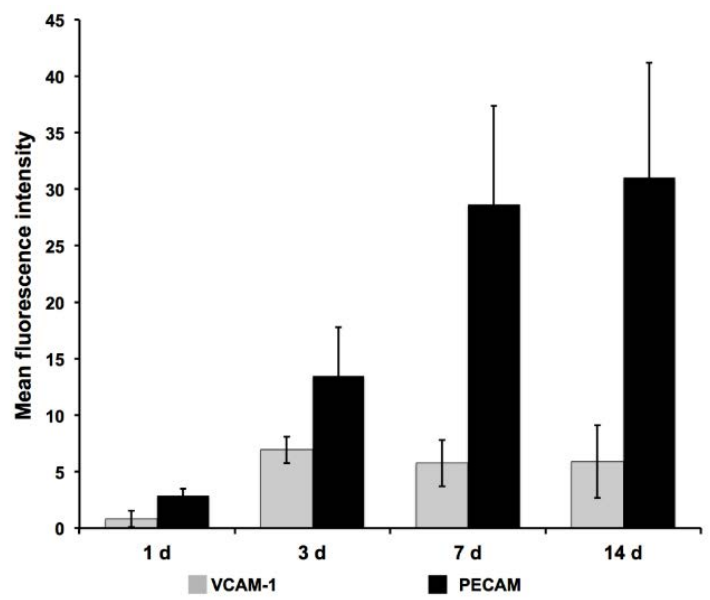

Supplemental Figure II. Mean fluorescence intensity measurement of VCAM-1 and PECAM-1 expression after endothelial denudation in carotid arteries.

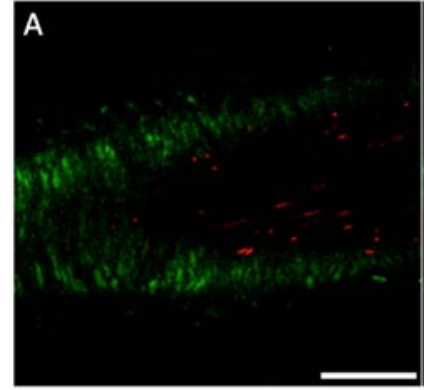

10 sec post injection

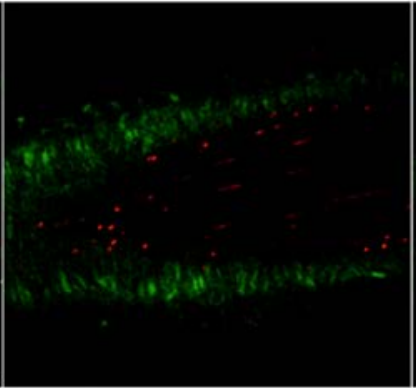

30 sec post injection

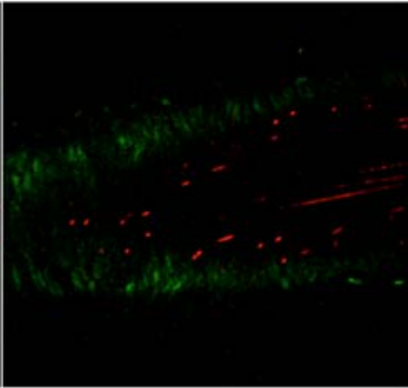

1 min post injection

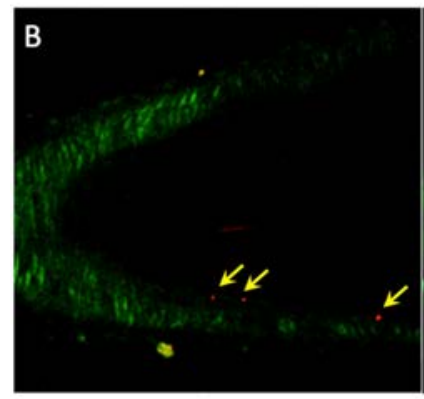

5 min post injection

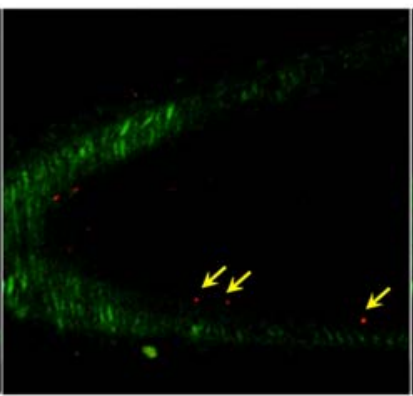

5 min 30 sec post injection

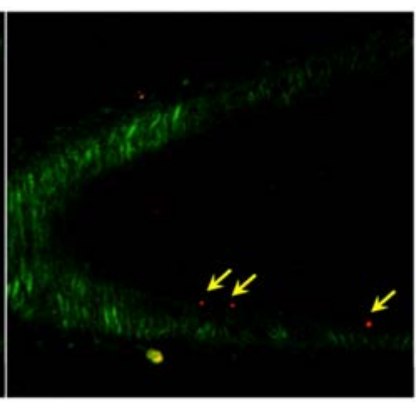

$6 \mathrm{~min}$ post injection

Supplemental Figure III. In vivo TPLSM images of wire-injured carotid arteries at $\mathbf{3} \mathbf{d}$ post surgery. A) Directly after the injection of $8 \times 10^{7} \mathrm{MB}_{\mathrm{VCAM}-1}$, the $\mathrm{MB}$ circulate in the vessel lumen. $\mathrm{B}$ ) Five minutes after $M_{V \text { VAM-1 }}$ injection, circulating $M_{\mathrm{VCAM}^{-1}}$ are cleared out and only $\mathrm{MB}_{\mathrm{VCAM}-1}$ (yellow arrows) bound to the injured vascular wall remain. For orientation, cells in adventitia and media are stained with SYTO 41 (green). MBVCAM-1 are labeled with rhodamine (red). Scale bar $=100 \mu \mathrm{m}$. 
12w diet + Wire injury
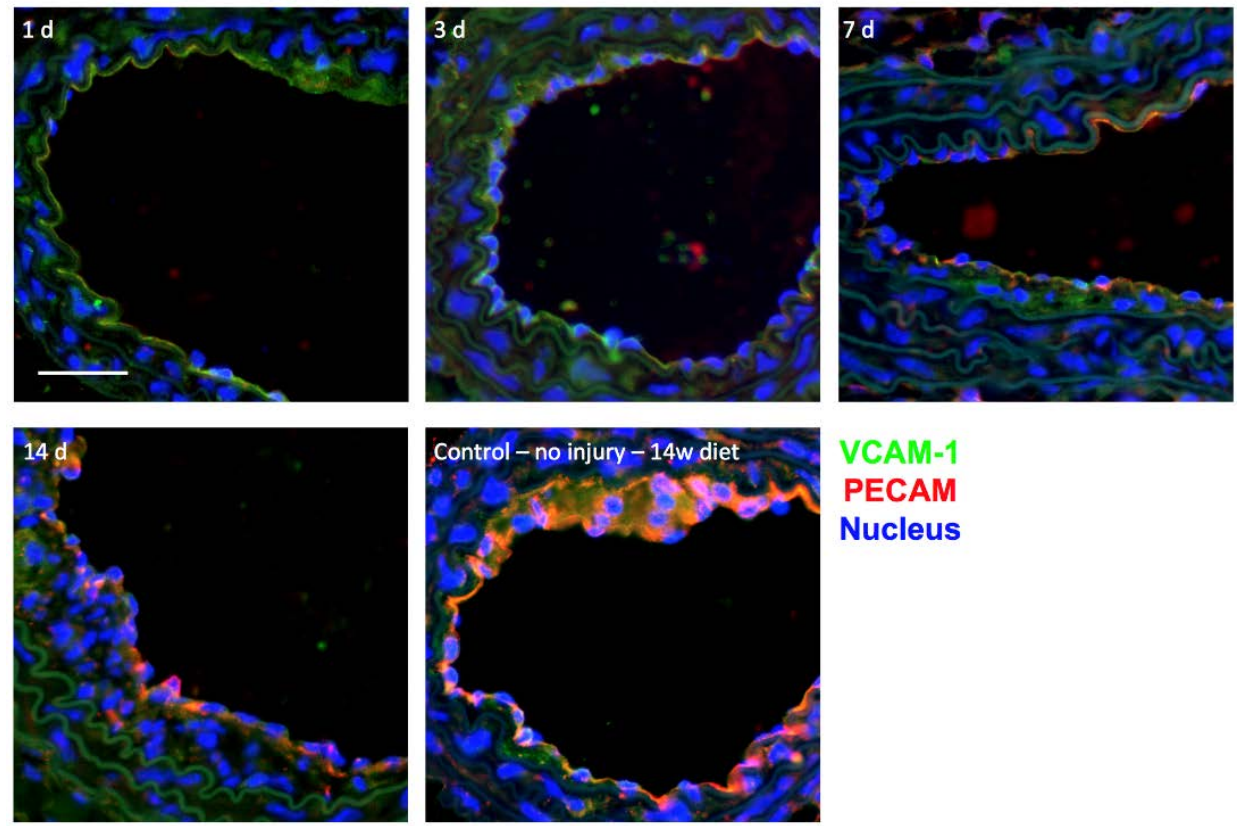

VCAM-1

PECAM

Nucleus

Supplemental Figure IV. Immunofluorescence staining of wire-injured carotids after 12 weeks of high cholesterol diet. Scale bar $=50 \mu \mathrm{m}$. 


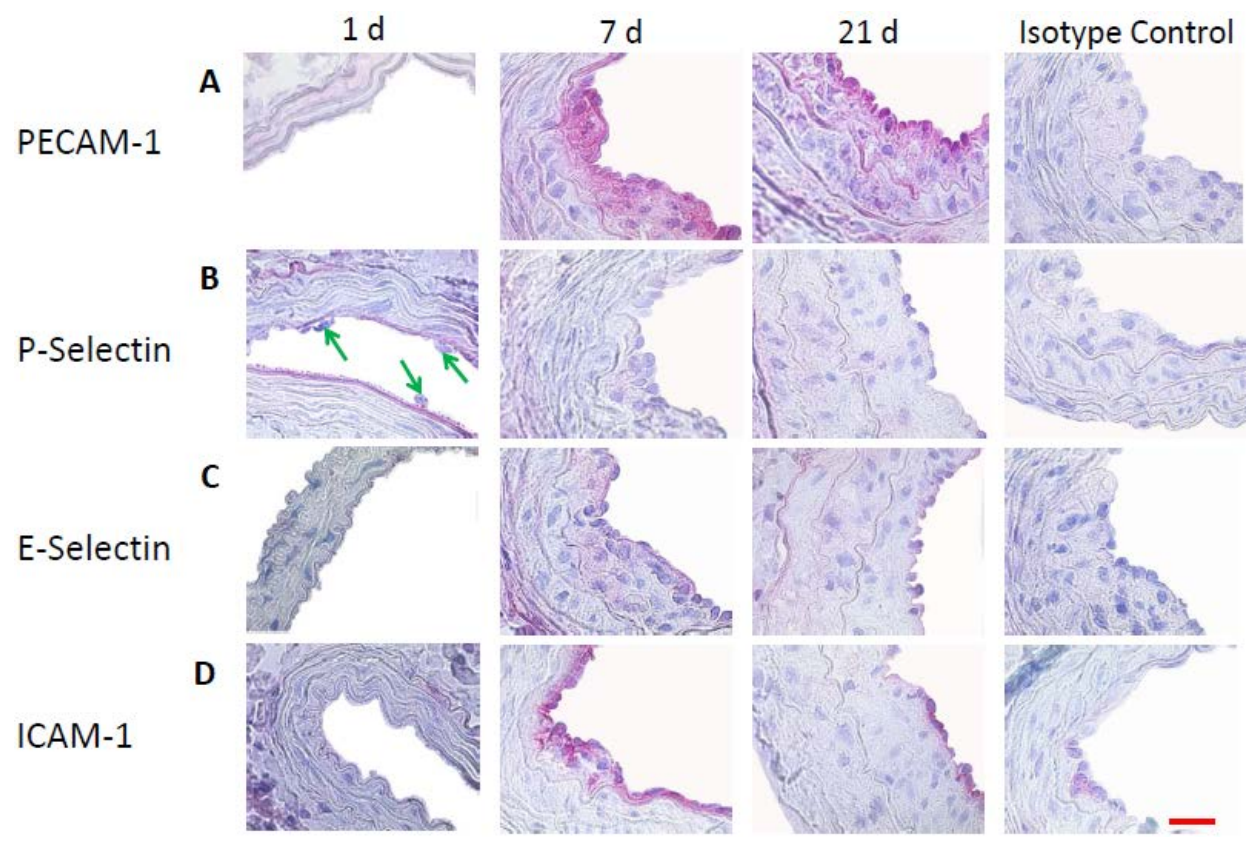

Supplemental Figure V. Immunohistochemistry of wire-injured carotid arteries. One day after arterial injury, PECAM-1, E-Selectin and ICAM-1 staining showed no expression of these markers on the luminal side of the artery ( $A, C$ and $D)$. P-Selectin could be detected on thrombocytes covering the denudated artery at $1 \mathrm{~d}$, but hardly on the remaining EC (green arrows). At 7 and $21 \mathrm{~d}$, the endothelial layer expresses high levels of PECAM-1 and ICAM-1 ( $A$ and D), moderate levels of ESelectin (C), but hardly any P-Selectin (B). Scale bar $=20 \mu \mathrm{m}$.

Supplemental Table I: Summary of the findings in literature about arterial endothelial and subendothelial marker expression.

\begin{tabular}{|c|c|c|c|}
\hline Markers & $\begin{array}{c}\text { Subendothelial } \\
\text { expression }\end{array}$ & $\begin{array}{c}\text { Activated } \\
\text { endothelial } \\
\text { expression }\end{array}$ & $\begin{array}{c}\text { Constitutional } \\
\text { endothelial } \\
\text { expression }\end{array}$ \\
\hline PECAM-1 & -21 & $+21,22$ & $+{ }^{21,22}$ \\
\hline ICAM-1 & $+46 *$ & $+15,22$ & $+{ }^{15,22}$ \\
\hline VCAM-1 & +46 & $+10,11,22$ & $-11,22$ \\
\hline E-Selectin & -24 & +22 & +22 \\
\hline P-Selectin & $-14,24$ & $+24 * *$ & +23 \\
\hline
\end{tabular}

* own expertise: almost no subendothelial expression of ICAM-1 was found (see Supplemental Figure V)

** own expertise: specific P-selectin signals could not conclusively be detected on remaining endothelial cells but only on attached platelets (see Supplemental Figure V) 


\section{References}

1. Sternberg K, Grabow N, Petersen S, et al. Advances in coronary stent technology--active drug-loaded stent surfaces for prevention of restenosis and improvement of biocompatibility. Curr Pharm Biotechnol. 2013;14(1):7690.

2. Lal BK. Recurrent carotid stenosis after CEA and CAS: diagnosis and management. Semin Vasc Surg. 2007;20(4):259-266.

3. Napoli C, de Nigris F, Williams-Ignarro S, Pignalosa O, Sica V, Ignarro LJ. Nitric oxide and atherosclerosis: an update. Nitric Oxide. 2006;15(4):265279.

4. Otsuka F, Finn AV, Yazdani SK, Nakano M, Kolodgie FD, Virmani R. The importance of the endothelium in atherothrombosis and coronary stenting. Nat Rev Cardiol. 2012;9(8):439-453.

5. Chaabane C, Otsuka F, Virmani R, Bochaton-Piallat ML. Biological responses in stented arteries. Cardiovasc Res. 2013;99(2):353-363.

6. Seedial SM, Ghosh S, Saunders RS, et al. Local drug delivery to prevent restenosis. J Vasc Surg. 2013;57(5):1403-1414.

7. Siddiqi OK, Faxon DP. Very late stent thrombosis: current concepts. Curr Opin Cardiol. 2012;27(6):634-641.

8. Inoue T, Croce K, Morooka T, Sakuma M, Node K, Simon DI. Vascular inflammation and repair: implications for re-endothelialization, restenosis, and stent thrombosis. JACC Cardiovasc Interv. 2011;4(10):1057-1066.

9. Grote K, Sonnenschein K, Kapopara PR, et al. Toll-Like Receptor 2/6 Agonist Macrophage-Activating Lipopeptide-2 Promotes Reendothelialization and Inhibits Neointima Formation After Vascular Injury. Arterioscler Thromb Vasc Biol. 2013;33(9):2097-2104.

10. Simsekyilmaz S, Cabrera-Fuentes HA, Meiler S, et al. Role of extracellular RNA in atherosclerotic plaque formation in mice. Circulation. 2014;129(5):598-606.

11. Lindner V, Collins T. Expression of NF-kappa B and I kappa B-alpha by aortic endothelium in an arterial injury model. Am J Pathol. 1996;148(2):427-438. 
12. Fokong S, Siepmann M, Liu Z, Schmitz G, Kiessling F, Gatjens J. Advanced characterization and refinement of poly $\mathrm{N}$-butyl cyanoacrylate microbubbles for ultrasound imaging. Ultrasound in medicine \& biology. 2011;37(10):16221634.

13. Fokong S, Theek B, Wu Z, et al. Image-guided, targeted and triggered drug delivery to tumors using polymer-based microbubbles. J Control Release. 2012;163(1):75-81.

14. Schober A, Zernecke A, Liehn EA, et al. Crucial role of the CCL2/CCR2 axis in neointimal hyperplasia after arterial injury in hyperlipidemic mice involves early monocyte recruitment and CCL2 presentation on platelets. Circ Res. 2004;95(11):1125-1133.

15. Wu Z, Curaj A, Fokong S, et al. Rhodamine-loaded intercellular adhesion molecule-1-targeted microbubbles for dual-modality imaging under controlled shear stresses. Circulation. Cardiovascular imaging. 2013;6(6):974-981.

16. Kiessling F, Bzyl J, Fokong S, Siepmann M, Schmitz G, Palmowski M. Targeted ultrasound imaging of cancer: an emerging technology on its way to clinics. Curr Pharm Des. 2012;18(15):2184-2199.

17. Kokzera P, Wu Z, Fokong S, et al. Fluorescently labeled microbubbles for facilitating translational molecular ultrasound studies. Drug Delivery and Translational Research. 2012;2(1):56-64.

18. Rademakers T, Douma K, Hackeng TM, et al. Plaque-associated vasa vasorum in aged apolipoprotein E-deficient mice exhibit proatherogenic functional features in vivo. Arterioscler Thromb Vasc Biol. 2013;33(2):249256.

19. Curcio A, Torella D, Indolfi C. Mechanisms of smooth muscle cell proliferation and endothelial regeneration after vascular injury and stenting: approach to therapy. Circ J. 2011;75(6):1287-1296.

20. Tahir H, Bona-Casas C, Hoekstra AG. Modelling the effect of a functional endothelium on the development of in-stent restenosis. PLoS One. 2013;8(6):e66138.

21. Ilan N, Madri JA. PECAM-1: old friend, new partners. Current opinion in cell biology. 2003;15(5):515-524. 
22. Davies MJ, Gordon JL, Gearing AJ, et al. The expression of the adhesion molecules ICAM-1, VCAM-1, PECAM, and E-selectin in human atherosclerosis. J Pathol. 1993;171(3):223-229.

23. Zeiffer U, Schober A, Lietz M, et al. Neointimal smooth muscle cells display a proinflammatory phenotype resulting in increased leukocyte recruitment mediated by P-selectin and chemokines. Circ Res. 2004;94(6):776-784.

24. Kennedy S, McPhaden AR, Wadsworth RM, Wainwright CL. Correlation of leukocyte adhesiveness, adhesion molecule expression and leukocyteinduced contraction following balloon angioplasty. $\mathrm{Br} \mathrm{J}$ Pharmacol. 2000;130(1):95-103.

25. Cybulsky MI, liyama K, Li H, et al. A major role for VCAM-1, but not ICAM-1, in early atherosclerosis. J Clin Invest. 2001;107(10):1255-1262.

26. Kaufmann BA, Carr CL, Belcik JT, et al. Molecular imaging of the initial inflammatory response in atherosclerosis: implications for early detection of disease. Arteriosclerosis, thrombosis, and vascular biology. 2010;30(1):5459.

27. Khanicheh E, Mitterhuber M, Xu L, Haeuselmann SP, Kuster GM, Kaufmann BA. Noninvasive ultrasound molecular imaging of the effect of statins on endothelial inflammatory phenotype in early atherosclerosis. PLoS One. 2013;8(3):e58761.

28. Khanicheh E, Qi Y, Xie A, et al. Molecular imaging reveals rapid reduction of endothelial activation in early atherosclerosis with apocynin independent of antioxidative properties. Arterioscler Thromb Vasc Biol. 2013;33(9):21872192.

29. Qu $\mathrm{Y}$, Shi $\mathrm{X}$, Zhang $\mathrm{H}$, et al. VCAM-1 siRNA reduces neointimal formation after surgical mechanical injury of the rat carotid artery. J Vasc Surg. 2009;50(6):1452-1458.

30. Nakashima Y, Raines EW, Plump AS, Breslow JL, Ross R. Upregulation of VCAM-1 and ICAM-1 at atherosclerosis-prone sites on the endothelium in the ApoE-deficient mouse. Arterioscler Thromb Vasc Biol. 1998;18(5):842851.

31. Bobryshev YV, Lord RS, Rainer SP, Munro VF. VCAM-1 expression and network of VCAM-1 positive vascular dendritic cells in advanced 
atherosclerotic lesions of carotid arteries and aortas. Acta Histochem. 1996;98(2):185-194.

32. Hastings NE, Feaver RE, Lee MY, Wamhoff BR, Blackman BR. Human IL-8 regulates smooth muscle cell VCAM-1 expression in response to endothelial cells exposed to atheroprone flow. Arterioscler Thromb Vasc Biol. 2009;29(5):725-731.

33. Seitz O, Schurmann $\mathrm{C}$, Hermes $\mathrm{N}$, et al. Wound healing in mice with high-fat diet- or ob gene-induced diabetes-obesity syndromes: a comparative study. Exp Diabetes Res. 2010;2010:476969.

34. Nascimento AP, Costa AM. Overweight induced by high-fat diet delays rat cutaneous wound healing. Br J Nutr. 2006;96(6):1069-1077.

35. Marin C, Ramirez R, Delgado-Lista J, et al. Mediterranean diet reduces endothelial damage and improves the regenerative capacity of endothelium. Am J Clin Nutr. 2011;93(2):267-274.

36. Roelke LH, Rodrigues SL, Lotufo PA, Mill JG. Correlation between the intima-media thickness of the proximal and distal common carotids. Arq Bras Cardiol. 2013;101(3):211-216.

37. Clevert DA, Sommer WH, Helck A, Reiser M. Duplex and contrast enhanced ultrasound (CEUS) in evaluation of in-stent restenosis after carotid stenting. Clin Hemorheol Microcirc. 2011;48(1):199-208.

38. Bauer M, Caviezel S, Teynor A, Erbel R, Mahabadi AA, Schmidt-Trucksass A. Carotid intima-media thickness as a biomarker of subclinical atherosclerosis. Swiss Med Wkly. 2012;142:w13705.

39. Bettinger $\mathrm{T}$, Bussat $\mathrm{P}$, Tardy $\mathrm{I}$, et al. Ultrasound molecular imaging contrast agent binding to both E- and P-selectin in different species. Invest Radiol. 2012;47(9):516-523.

40. Deshpande N, Lutz AM, Ren $\mathrm{Y}$, et al. Quantification and monitoring of inflammation in murine inflammatory bowel disease with targeted contrastenhanced US. Radiology. 2012;262(1):172-180.

41. Fokong S, Fragoso A, Rix A, et al. Ultrasound Molecular Imaging of ESelectin in Tumor Vessels Using Poly n-Butyl Cyanoacrylate Microbubbles Covalently Coupled to a Short Targeting Peptide. Invest Radiol. 2013. 
42. Bachawal SV, Jensen KC, Lutz AM, et al. Earlier detection of breast cancer with ultrasound molecular imaging in a transgenic mouse model. Cancer Res. 2013;73(6):1689-1698.

43. Tardy I, Pochon S, Theraulaz M, et al. Ultrasound molecular imaging of VEGFR2 in a rat prostate tumor model using BR55. Invest Radiol. 2010;45(10):573-578.

44. Wang $\mathrm{H}$, Machtaler $\mathrm{S}$, Bettinger $\mathrm{T}$, et al. Molecular imaging of inflammation in inflammatory bowel disease with a clinically translatable dual-selectintargeted US contrast agent: comparison with FDG PET/CT in a mouse model. Radiology. 2013;267(3):818-829.

45. Davidson BP, Kaufmann BA, Belcik JT, Xie A, Qi Y, Lindner JR. Detection of antecedent myocardial ischemia with multiselectin molecular imaging. J Am Coll Cardiol. 2012;60(17):1690-1697.

46. Cai $Q$, Lanting L, Natarajan R. Interaction of monocytes with vascular smooth muscle cells regulates monocyte survival and differentiation through distinct pathways. Arteriosclerosis, thrombosis, and vascular biology. 2004;24(12):2263-2270. 



\section{Chapter 5}

\section{Molecular ultrasound imaging of \\ JAM-A depicts acute alterations in blood flow and early endothelial dysregulation}

Adelina Curaj, Zhuojun Wu, Marc van Zandvoort, Oliver Gresch, Christian Weber, Twan Lammers, Elisa A. Liehn, Fabian Kiessling Under revision 


\section{Abstract}

Objective: The Junctional Adhesion Molecule (JAM-A) was evaluated as a target for molecularly-targeted ultrasound imaging (molUS) of transient endothelial dysfunction under acute blood flow variations.

Background: JAM-A is a protein physiologically located in inter-endothelial tight junctions, and it focally redistributes to the luminal surface of blood vessels under abnormal shear and flow conditions accompanying atherosclerotic lesion development.

Methods: Endothelial dysfunction was induced in $\mathrm{ApoE}^{-1-}$ mice by carotid partialligation (PL) and wire-injury (WI). JAM-A expression was investigated by molUS using antibody-targeted poly(n-butyl cyanoacrylate) microbubbles (MBJAM-A) and validated with immunohistology. Flow disturbance and arterial remodeling were assessed using functional ultrasound imaging.

Results: PL led to an immediate drop in perfusion at the ligated side and a direct compensatory increase at the healthy side. This was accompanied by a strongly increased JAM-A expression and MBJAM-A binding at the PL side, and a moderate and temporarily increase in the contralateral artery $(\approx 14$ times, $p<0.001$ and $\approx 5$ times, $p<0.001$ respectively, higher than control). WI induced slow plaque growth and slowly decreasing perfusion. In the contralateral artery, perfusion adapted accordingly. Interestingly, only at the WI side increased JAM-A expression and MBJAM-A binding was found while the slow flow changes at the contralateral side did not induce any effects.

Conclusions: Temporary blood flow variations induce an endothelial rearrangement of JAM-A that can be visualized using MBJAM-A. Thus, JAM-A may be considered as marker of acute endothelial activation and dysfunction. Its imaging may facilitate the early detection of cardiovascular risk areas and enables the therapeutic prevention of their progression towards an irreversible pathological state. 


\section{Condensed abstract}

The loss or alteration of endothelial regulatory function is a major risk factor for the development of atherosclerosis and myocardial infarction. The present study shows that arterial blood flow fluctuations determine temporary endothelial dysfunction, inducing JAM-A translocation to the endothelial surface, which can sensitively be detected by molecularly-targeted ultrasound imaging employing JAM-A-targeted microbubbles. Our results suggest JAM-A as a marker of acute endothelial activation and it may be suitable to assess the risk of patients with oscillatory arterial blood pressure to develop atherosclerotic lesions and thrombosis.

\section{Introduction}

The initial hallmark of atherosclerosis is endothelial dysfunction. Vessel bifurcations and curvatures ${ }^{1}$ are predilection sites for the development of atherosclerotic lesions due to natural disturbance of laminar flow ${ }^{2}$. Here, molecular changes in the endothelium may indicate whether alterations in perfusion are of pathological relevance ${ }^{3}$. Therefore, the identification of specific early stage biomarkers in combination with an easy accessible, radiation-free, noninvasive, and cheap diagnostic method is of significant interest.

Advances in molecular targeting ${ }^{4,5}$ of endothelial lesions in big and small arteries have been achieved using different diagnostic probes recognizing activated endothelial cells ${ }^{6-8}$. In this context, molecular ultrasound imaging (molUS) $)^{9}$ was introduced as an extension of conventional ultrasound (US) imaging, which is capable of quantifying flow velocity and back flow within major arteries, providing anatomical and functional information, but which lacks visualization at the molecular level ${ }^{10}$. Prominent inflammation biomarkers such as ICAM-1 ${ }^{11}$, VCAM$1^{7,8,12}$, P-Selectin ${ }^{11}$ and $\alpha_{v} \beta_{3}{ }^{13}$, have already been evaluated for molecular diagnosis of different stages of atherosclerosis by employing targeted radiotracers, liposomes and MB. However, early detection of active endothelial 
sites remains challenging for many targets due to their constitutive luminal availability ${ }^{11,14,15}$, transient upregulation ${ }^{11,14,15}$, target $\operatorname{access}^{16}$, or partial solubility ${ }^{17}$.

JAM-A, has been recently involved in molecular imaging protocols employing targeted USPIOs and $\mathrm{MB}^{18}$. JAM-A proved to be the biological marker most responsive to acute changes in blood flow ${ }^{19}$, being up-regulated specifically on endothelial cells at the flow-dependent predilection sites of atherosclerosis ${ }^{20}$, which are responsible for monocyte recruitment and accumulation in the vascular wall ${ }^{19}$. Junctional adhesion molecules are members of an immunoglobulin subclass, which under non-pathological conditions are located in the tight junctions of endothelial and epithelial cells ${ }^{21}$, inaccessible from the lumen. Recent studies have shown redistribution of JAM-A to the luminal surface upon inflammatory stimuli and endothelial activation, and have uncovered a pivotal role for JAM-A in leukocyte recruitment and transmigration ${ }^{22}$.

The aim of our study was to evaluate JAM-A as a target for molUS of transient endothelial activation under acute blood flow variations. Therefore, we used JAMA-targeted poly(n-butyl cyanoacrylate) microbubbles (MBJAM-A) as a contrast agent for molUS imaging in two mouse models of endothelial activation, wireinjury and partial-ligation, both leading to atherosclerotic plaque development23. We found that MBJAM-A bind specifically to JAM-A on activated endothelium and are able to identify the location of areas with endothelial dysfunction and early plaque development. Furthermore, our data indicate that not only flow reduction due to vessel obstruction, but also flow increase due to compensatory blood flow redistribution in the contralateral carotid, induce luminal exposure of JAM-A, both illustrating the high sensitivity of this marker for assessing acute vascular vulnerability and remodeling.

\section{Materials and methods}

\section{Microbubble synthesis and labeling}


Poly(n-butyl cyanoacrylate) (PBCA) MB were synthesized as described previously ${ }^{11,23}$. For functionalization, MBs were coated with streptavidin then mixed with biotinylated anti-JAM-A antibodies (clone H202-106, AbD Serotec). After 10 minutes incubation, conjugated MBs were separated from excess antibody by 30 minutes flotation, then re-suspended in $50 \mu \mathrm{L}$ HEPES/Triton buffer and prepared for injection. Prior to the intravenous (i.v.) injection, $50 \mu \mathrm{L} 0,9 \%$ saline solution was added to the MBs.

\section{Atherogenic murine model of endothelial dysfunction and arterial remodeling}

All animal experiments were approved by local authorities (LANUV AZ: 84.02.04.2012.A161). Two animal models $(n=87)$ of induced endothelial dysfunction followed by accelerated atherosclerosis were used in this study:

1. Partial-ligation $(P L)$ model $(n=44)$ : to induce a flow-induced atherosclerotic lesion, three out of four branches of the left carotid artery (LCA), the left external and internal carotid arterial branches, and occipital artery, were surgically isolated and ligated, diverting the blood flow solely through the superior thyroid artery. Plaque development was analyzed 1, 2, 3, and 4 weeks ( $n=3-5$ each) after the intervention; 2 . Wire-injury (WI) model $(n=43)$ : arterial denudation was performed using a coated guide wire as previously described ${ }^{16}$. The wire-induced arterial injury and neointima formation was analyzed at 1, 4, 7, 14 and 21 days $(n=3-5$ each) after the procedure. For this, female 8-week-old female apolipoprotein knockout (ApoE-/-) mice were fed an atherogenic diet $(21 \%$ fat, $0.15 \%$ cholesterol, 19.5\% casein; Altromin, Lage, Germany) for 1 week before and 3 and 4 weeks after the intervention, respectively.

\section{In vivo molecular and functional US imaging}

Both animal models were investigated by functional and molUS imaging at different time points $(1,2,3,4$ weeks $(n=24)$ and 1, 4, 7, 14 and 21 days $(n=23)$, respectively). Imaging was performed under anesthesia with $1 \%$ isoflurane 
having the mice placed on a heated table (Vevo Mouse Handling Table) ${ }^{8,11}$. In vivo functional US (funcUS) imaging was performed at first. B-, M- and PW-mode images of both the injured carotids and the contralateral arteries were recorded using a 40MHz US probe on the Vevo770 device (Visualsonics, Toronto). Carotid arteries were imaged in the long axis, having the bifurcation in the field of view. M-mode images were used to quantify the arterial wall thickness. PW-mode data were recorded covering the whole common carotid enabling the assessment of blood flow at i) the bifurcation, ii) arteries' origin and iii) in between. Subsequently, analysis was performed for all 3 sites and mean values are reported.

In vivo molUS measurements were performed as previously described8,11. For competitive binding studies $(n=3)$ at 2 weeks after PL, a 20-fold higher concentration of free anti-JAM-A antibody $(100 \mu \mathrm{g})$ was injected i.v. to each mouse 10 minutes before MBJAM-A administration to block JAM-A binding sites. Seven minutes after MB injection, molUS imaging in contrast mode (Figure 1A) was performed as previously described8,11 to measure the difference in the signal levels before and directly after a destructive pulse24 (Figure 1B).

A

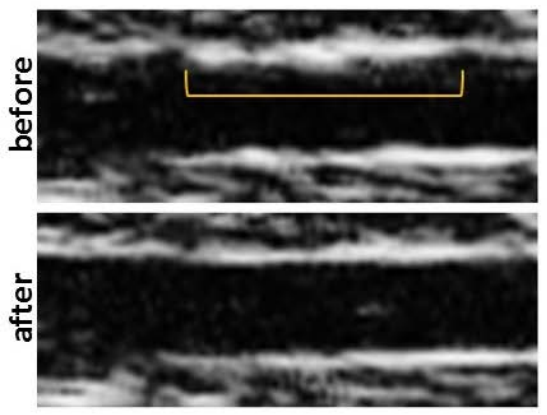

B

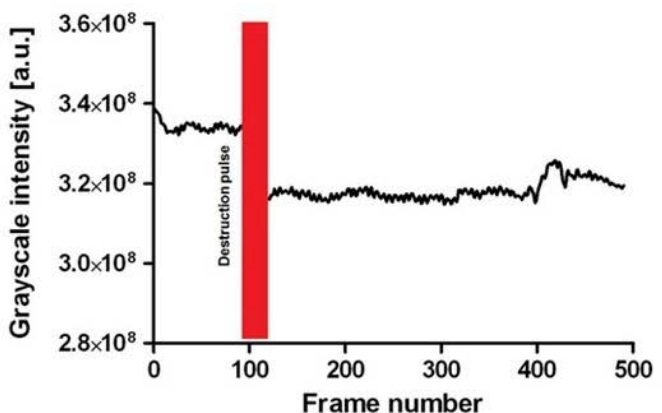

Figure 1. In vivo molUS imaging of carotid arteries subjected to partial-ligation. A) Representative in vivo US images of LCA in the long axis imaging plane before the destructive pulse (upper panel), where the region with $\mathrm{MB}_{\mathrm{JAM}-\mathrm{A}}$ attached to their target on the luminal side of the artery is indicated with orange line. After applying the destructive pulse, $\mathrm{MB}_{\text {JAM-A }}$ are destroyed and cannot be visualized anymore (lower panel). B) An example of a destruction-replenishment curve obtained after post-processing the contrast mode molUS images. Circulation time of MB JAM-A before the destructive pulse was 7 minutes. 


\section{Immunohistology}

At the end of each experiment, mice ( $n=4$ per animal model and time point) were euthanized by intraperitoneal overdose $(400 \mathrm{gm} / \mathrm{kg}$ Ketamine and $40 \mathrm{mg} / \mathrm{kg}$ Xylazine) injection of anesthetic, followed by terminal eye bleeding. Intracardial perfusion with PBS was performed to remove the remaining blood. Then, the common carotid arteries $(6-7 \mathrm{~mm})$ were excised, embedded in Tissue-Tek O.C.T. (4583, Sakura) and snap-frozen. Serial tissue sections $(5 \mu \mathrm{m})$ starting with the bifurcation were obtained from isolated carotids and stained for JAM-A (Serotec) and PECAM-1 (sc-1506) expression. Fluorescein streptavidin (SA5001, Vector Laboratories), and Cy5 conjugated secondary antibodies, respectively, were used for visualization. Sections were analyzed using Diskus software (Hilgers, Germany).

\section{Detection of serum markers}

Quantification of cytokines IL-1 $\beta$, IL-2, IL-4, IL-5, IL-6, IL-10, IL-12, IL-13, IL-17A, IFN- $\gamma$, TNF- $\alpha$ and GM-CSF in murine plasma samples with 1:2 sample dilution was conducted using the LUNARIS Mouse 12-Plex Cytokine Kit from AYOXXA Biosystems (Cat. No. LMC-20121S). The assay was performed according to the manufacturers' instructions.

\section{Statistical analysis}

Differences between groups of ex vivo and in vivo $\mathrm{MB}$ adhesion assays were analyzed using 1-way ANOVA followed by Newman-Keuls post-hoc-test. Pvalues of $<0.05$ were considered significant. Error bars shown on graphs represent the standard deviation. Statistical analysis was performed using GraphPad Prism 6.1 (GraphPad Software). 


\section{Results}

\section{MolUS imaging of JAM-A expression in carotid arteries in the PL-model}

The specificity of MBJAM-A binding was confirmed 2 weeks after PL by competitive binding experiments using a 20 -fold higher concentration of free anti-JAM-A antibody administered before MBJAM-A injection. Compared to normal binding conditions, blocking JAM-A lead to a reduction of MBJAM-A binding by $94 \%$ $(p=0.0008)$.

The longitudinal assessment of JAM-A expression by molUS revealed a slightly enhanced binding of MBJAM-A to the luminal side of the LCA at 1 week post-PL, followed by a strong increase after 2 weeks. Subsequently, MBJAM-A binding decreased to a stable level 3 and 4 weeks post-ligation (Figure 2A). Interestingly, JAM-A upregulation was also detected in the contralateral carotid 2 weeks after $\mathrm{PL}$, followed by a significant decrease in MBJAM-A binding at the 3 weeks time point and a normalization 4 weeks post-intervention (Figure 2B).

\section{Immunohistology of carotid arteries in the PL-model}

In order to determine the expression pattern and localization of JAM-A during endothelial activation and plaque development, JAM-A and PECAM-1 were stained at 1, 2, 3 and 4 weeks post-surgery (Figure 2C). One week after PL of the LCA, only focal areas of endothelia with JAM-A upregulation could be observed compared to sham animals. By week 2, JAM-A expression drastically increased in both the endothelial and sub-endothelial layer, reaching a peak in luminal translocation.

Despite the lack of ligation (but in line with molUS) also in the right carotid artery (RCA) an upregulation and luminal exposure of JAM-A was found 2 weeks after ligation of the LCA (Figure 2D), which subsequently decreased reaching baseline levels 4 weeks post-surgery. 
A

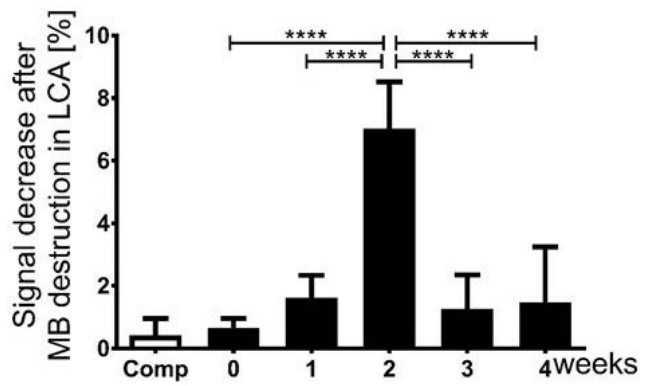

C

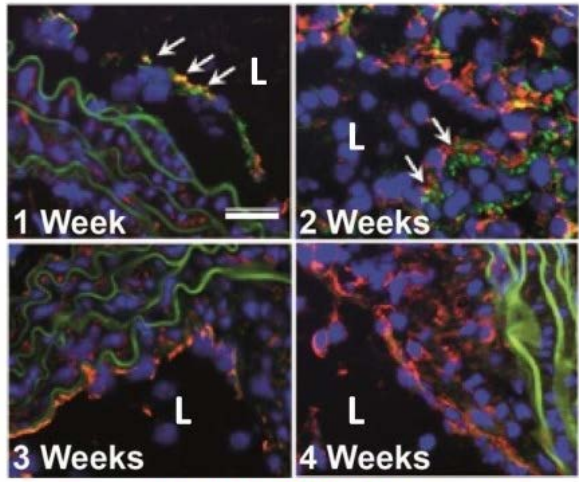

green - JAM-A; red - PECAM-1; blue - DAPI
B

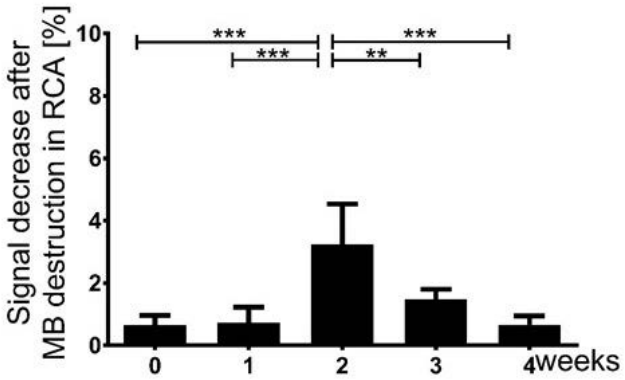

D

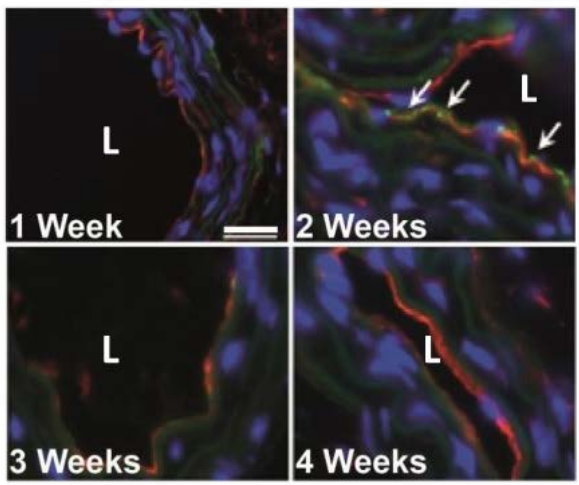

Figure 2. Quantification of JAM-A after partial-ligation. A) US data analysis of MB JAM-A $_{\text {A }}$ binding in the LCA ( $n=24)$ after PL compared with control (pre-operation) and competition (Comp) conditions.

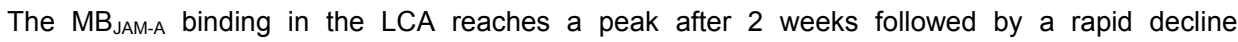
$\left({ }^{* * * *} p<0.001\right)$. B) The corresponding US signal detected in the RCA $(n=24)$ indicated also a transient increase of MB JAM-A binding 2 weeks post-PL, which was also in line with the immunohistology $\left({ }^{* *} p<0.01,{ }^{* * *} p<0.001\right)$. C) Immunohistological staining of JAM-A (green) in the LCA (arrows) ( $n=4$ per time point) shows the expression of JAM-A on the surface of endothelial cells (stained with PECAM-

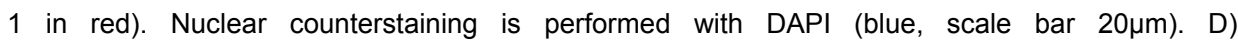
Immunohistological staining of JAM-A in the RCA ( $n=4 /$ time point) (scale bar $20 \mu \mathrm{m}$ ). L marks the arterial lumen.

\section{Morphological and functional measures of arterial remodeling in the PL-model}

In order to investigate the accompanying effects of JAM-A upregulation in both LCA and RCA, vascular wall thickness was quantified by US measurements. The vascular wall thickness in the LCA continuously increased over the examination 
period with most pronounced changes at the early time points (Figure 3A), indicating a progressive luminal stenosis (Figure 3B).

A

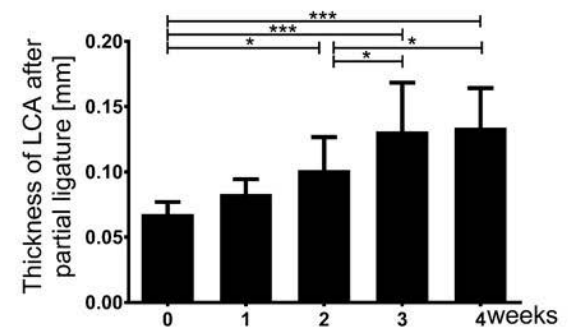

B

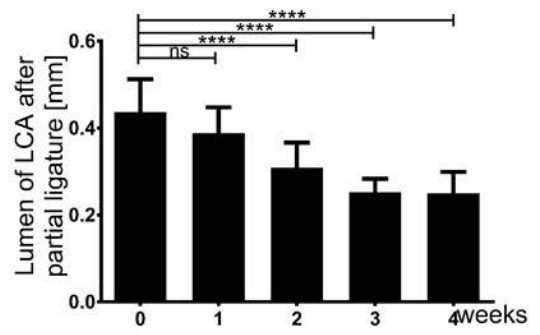

C

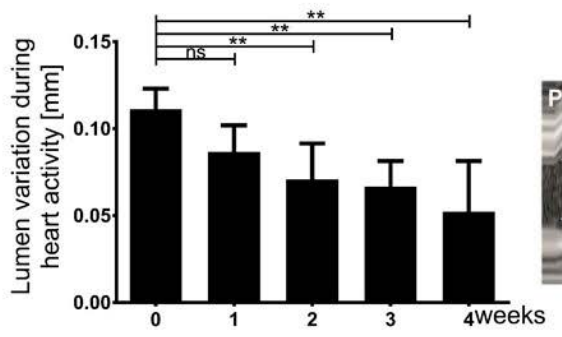

D

$\mathbf{E}$
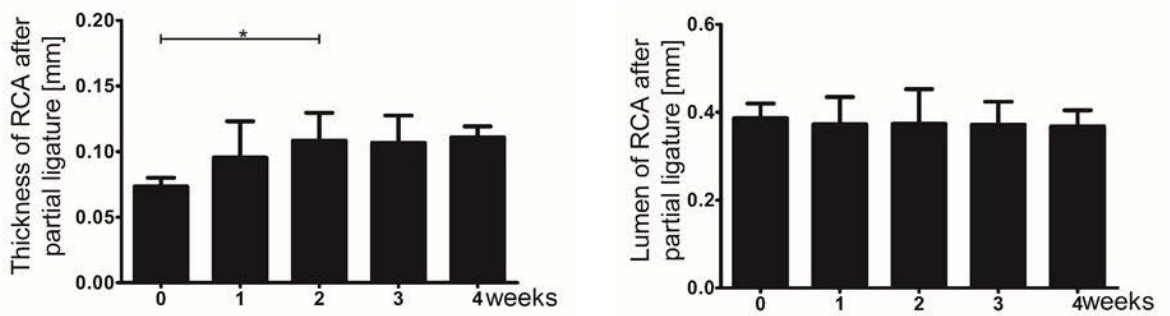

Figure 3. Arterial remodeling after partial-ligation. A) US quantification of vessel wall thickness $(n=21)$ revealed an initial significant increase 2 weeks after $P L$, which peaks at later time points $\left({ }^{*} p<0.05,{ }^{* * *} p<0.001\right)$. B) The early growth of an atherosclerotic plaque corresponds with a progressive luminal stenosis $\left({ }^{* * *} p<0.001\right)$. C) The elasticity of the vessel decreased progressively, along with the plaque development and arterial narrowing $\left({ }^{* *} p<0.01\right)$. Representative M-Mode images are shown, before surgery, 1 and 4 weeks post-surgery, as indicated. White arrows indicate the lumen variation during systole (S) and diastole (D). D) Also in the contralateral carotid artery $(n=21)$, an increase in thickness of the arterial wall was observed 2 weeks after PL $\left({ }^{*} p<0.05\right)$. However, the lumen of right carotid artery remained stable over the time of the experiment $E$ ). 
Along with the plaque development and arterial narrowing, a decreased elasticity was observed, as shown by the quantification of lumen variation during systole and diastole (Figure 3C).

Interestingly, also in the contralateral carotid a significant increase in arterial wall thickness was observed 2 weeks after PL, which, however, did not further progress at later time points (Figure $3 \mathrm{D}$ ). In addition, this increase was not associated with a narrowing of the vessel lumen (Figure 3E) or a change in arterial wall elasticity during heart activity (data not shown).

Blood flow assessed by Doppler US was reduced by 56 to $69 \%$ over the timespan of 4 weeks in the LCA after PL (Figure 4). As a compensatory response, the blood flow in the RCA increased reaching a stable value after 3 weeks, suggesting a hemodynamic adjustment of the blood flow after PL.

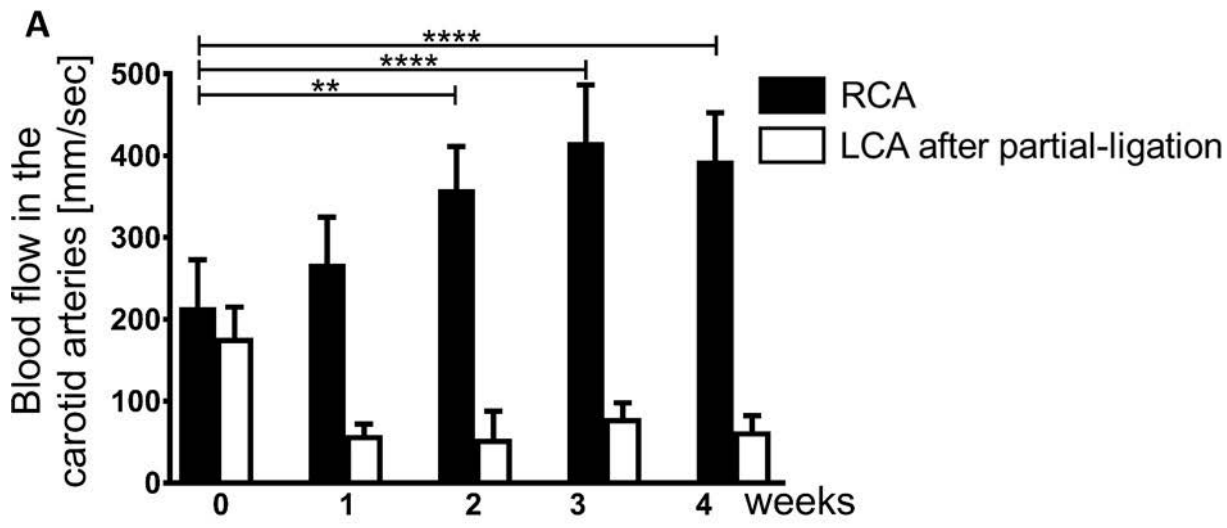

B
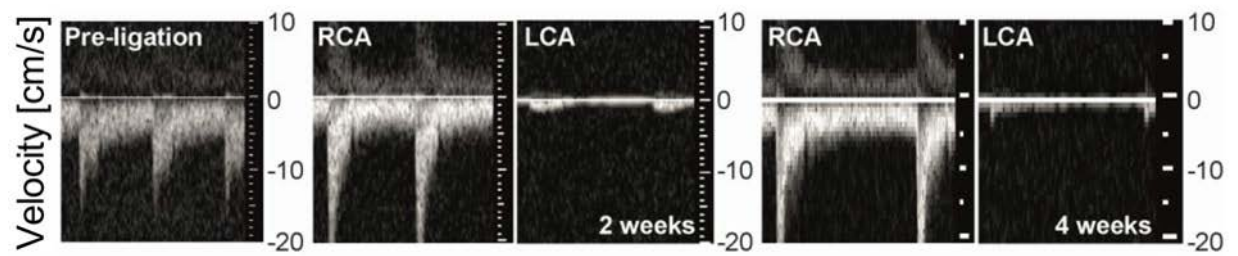

Figure 4. Blood flow hemodynamic after partial-ligation. A) Blood flow is reduced in the left carotid artery after partial-ligation (white bars), while blood flow in the right carotid artery adjusts progressively to a higher velocity, stabilizing after 3 weeks $\left({ }^{* *} p<0.01,{ }^{* * *} p<0.001\right)(n=21)$. B) Representative ultrasound images of flow velocity in carotid arteries before, as well as 2 weeks and 4 weeks after partial-ligation. 


\section{Inflammatory serum markers after PL}

To determine if hemodynamic changes induce systemic inflammation and vascular vulnerability, inflammation markers and immune cell content of blood samples were quantified at different time points. The absolute numbers of leukocytes, neutrophils, monocytes, and lymphocytes did not significantly vary during the experiments (Table 1). Moreover, general inflammatory and antiinflammatory markers, such as interferon (IFN) $\mathrm{\gamma}$, tumor necrosis factor (TNF) $\alpha$, granulocyte-macrophage colony-stimulating factor (GM-CSF), interleukin $1 \mathrm{~b}$, interleukin 2, interleukin 4, interleukin 5, interleukin 6, interleukin 10, interleukin 12, interleukin 13, and interleukin 17 did not show significant changes in serum of the mice at any time point after PL (Table 2), indicating the absence of general inflammatory condition.

Table 1. Immune cells in blood at different time points after partial ligature

\begin{tabular}{|l|l|l|l|l|l|l|}
\hline \multirow{2}{*}{ Cell type } & Before & \multicolumn{4}{c|}{ After partial ligature } & \multirow{2}{*}{ P Value } \\
\cline { 5 - 7 } & surgery & 1 week & 2 weeks & 3 weeks & 4 weeks & \\
\hline Leukocytes $\left(\times 10^{3} / \mu l\right)$ & $8.0 \pm 1.9$ & $6.5 \pm 1.2$ & $6.4 \pm 1.7$ & $7.9 \pm 1.4$ & $6.2 \pm 1.3$ & n.s. \\
\hline Neutrophils (\%) & $14 \pm 2.2$ & $18 \pm 6.7$ & $13 \pm 2.4$ & $17 \pm 3.9$ & $13 \pm 2.7$ & n.s. \\
\hline Monocytes (\%) & $4.8 \pm 1.3$ & $5.2 \pm 1.3$ & $4.7 \pm 0.9$ & $5.2 \pm 1.5$ & $3.0 \pm 1.4$ & n.s. \\
\hline Lymphocytes (\%) & $76 \pm 2.7$ & $71 \pm 5.5$ & $75 \pm 2.5$ & $72 \pm 4.1$ & $79 \pm 0.9$ & n.s. \\
\hline
\end{tabular}

\section{JAM-A expression and imaging in the WI-model}

To show that JAM-A is a sensitive target of endothelial activation and dysfunction, we induced a mechanic endothelial injury of the LCA, which lead to a local accelerated atherosclerotic plaque formation. MolUS imaging with MBJAM-A, demonstrated a transient increase of JAM-A on the luminal surface of the LCA, with a peak at 4 days after WI (Figure 5A). In contrast to the PL-model, no increased binding of MBJAM-A was found in the RCA (Figure 5B) although there was also a significant increase in blood flow (Figure 5D) and an increase in wall thickness over a period of 3 weeks (Figure 5E). 
Table 2. Serum markers at different time points after partial ligature

\begin{tabular}{|c|c|c|c|c|c|c|}
\hline \multirow{2}{*}{$\begin{array}{l}\text { Serum } \\
\text { marker }\end{array}$} & \multirow{2}{*}{$\begin{array}{l}\text { Before } \\
\text { surgery }\end{array}$} & \multicolumn{4}{|c|}{ After partial ligature } & \multirow{2}{*}{ P Value } \\
\hline & & 1 week & 2 weeks & 3 weeks & 4 weeks & \\
\hline$I F N-\mathrm{Y}$ & $0.2 \pm 0.3$ & $0.0 \pm 0.0$ & $1.7 \pm 3.4$ & $0.0 \pm 0.0$ & $0.0 \pm 0.1$ & n.s. \\
\hline$T N F-\alpha$ & $0.4 \pm 0.5$ & $0.1 \pm 0.1$ & $0.2 \pm 0.1$ & $0.5 \pm 0.9$ & $0.5 \pm 0.3$ & n.s. \\
\hline GM-CSF & $0.4 \pm 0.1$ & $0.3 \pm 0.1$ & $0.2 \pm 0.1$ & $0.5 \pm 0.3$ & $0.3 \pm 0.1$ & n.s. \\
\hline$I L 1 b$ & $5.0 \pm 8.1$ & $1.3 \pm 1.7$ & $3.0 \pm 4.9$ & $13.9 \pm 22.2$ & $3.7 \pm 7.4$ & n.s. \\
\hline$I L 2$ & $0.0 \pm 0.0$ & $0.0 \pm 0.0$ & $0.0 \pm 0.0$ & $0.9 \pm 1.9$ & $0.1 \pm 0.3$ & n.s. \\
\hline IL 4 & $5.9 \pm 7.9$ & $0.0 \pm 0.0$ & $0.0 \pm 0.0$ & $12.8 \pm 25.6$ & $3.3 \pm 6.6$ & n.s. \\
\hline$I L 5$ & $1.8 \pm 1.9$ & $2.4 \pm 0.5$ & $1.3 \pm 0.8$ & $2.1 \pm 2.3$ & $3.2 \pm 4.3$ & n.s. \\
\hline$I L 6$ & $7.0 \pm 8.9$ & $7.3 \pm 6.3$ & $8.6 \pm 8.1$ & $15.8 \pm 24.7$ & $14.6 \pm 15.8$ & n.s. \\
\hline IL 10 & $7.9 \pm 8.5$ & $12.5 \pm 17.5$ & $2.7 \pm 4.9$ & $10.7 \pm 19.0$ & $16.0 \pm 21.3$ & n.s. \\
\hline IL 12 & $2.3 \pm 3.7$ & $0.9 \pm 1.6$ & $0.9 \pm 1.9$ & $8.7 \pm 12.2$ & $3.6 \pm 2.8$ & n.s. \\
\hline IL 13 & 3.3. \pm 6.6 & $5.0 \pm 10.0$ & $0.0 \pm 0.0$ & $24.2 \pm 48.4$ & $0.0 \pm 0.0$ & n.s. \\
\hline IL 17 & $3.6 \pm 4.4$ & $0.0 \pm 0.0$ & $0.0 \pm 0.0$ & $1.8 \pm 3.1$ & $3.6 \pm 4.9$ & n.s. \\
\hline
\end{tabular}

However, it is a major difference to the PL-model that in the WI-model, the vascular obstruction of the LCA occurs slowly, which is reflected by the continuously increasing wall thickness (Figure $5 \mathrm{C}$ ). The immunohistologically determined endothelial JAM-A expression was in strong agreement with the molUS data (Figure $5 \mathrm{~F}$ ). 
A

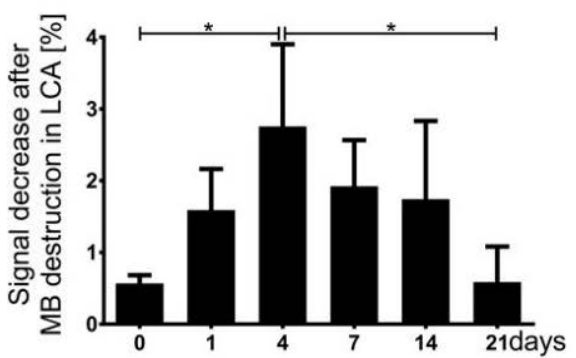

C

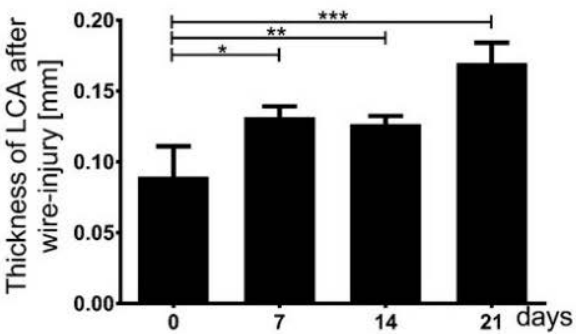

B

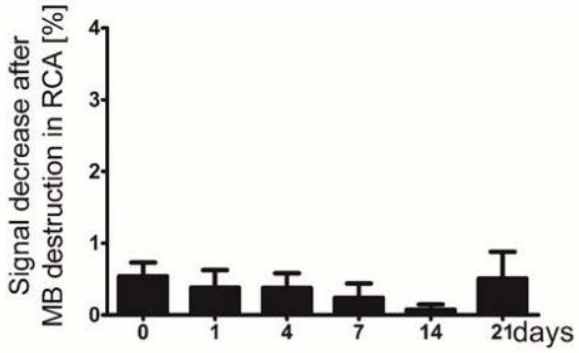

D

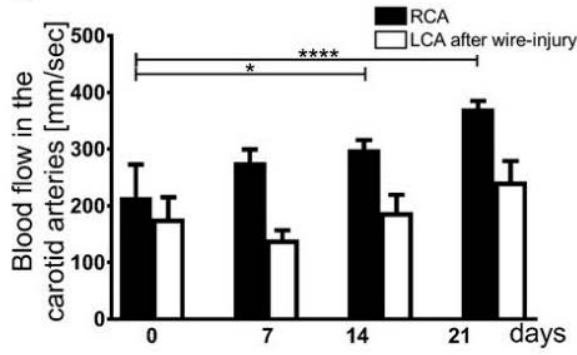

$\mathbf{E}$

$\mathbf{F}$
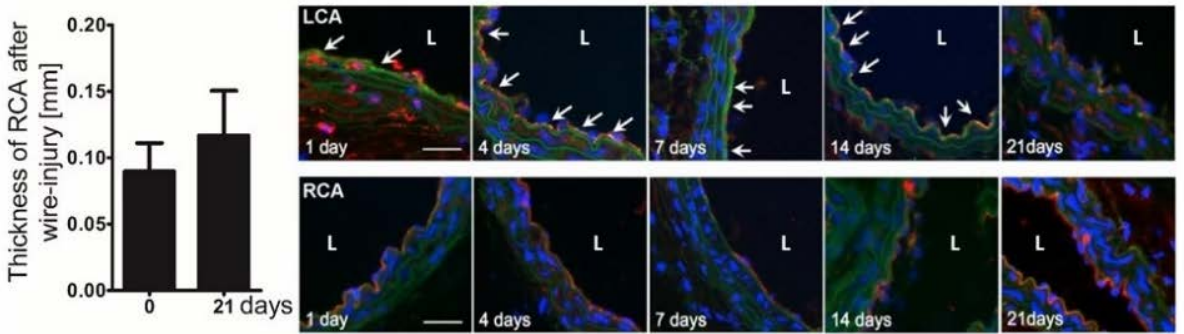

Figure 5. Quantification of JAM-A expression after wire-injury. A) US data analysis before and at indicated time points after WI using MB JAM-A in LCA ( $n=23)$. MB binding peaks after 4 days $\left({ }^{*} p<0.05\right)$. B) The RCA ( $n=23$ ) showed no significant changes during the experiment. C) US quantification of vessel wall thickness revealed an initial significant increase 7 days after $\mathrm{WI}$, which continues to grow until the 21 days after intervention $\left({ }^{*} p<0.05,{ }^{* *} p<0.01,{ }^{* * *} p<0.001\right)$. D) Blood flow is not significantly reduced in the LCA after WI (white bars), while blood flow in the RCA adjusts progressively to a higher velocity, being significantly increased after 14 days $\left({ }^{*} p<0.05\right.$, $\left.{ }^{* * * *} p<0.001\right)$. E) US quantification of vessel wall thickness of the RCA revealed an increase, but not significant, 21 days after intervention. F) Immunohistological staining of JAM-A (green, arrows) confirms the expression of JAM-A on the surface of endothelial cells (stained with PECAM-1 in red) in the LCA (upper panel) and its absence in the RCA (lower panel) ( $n=4$ per time point). Nuclear counterstaining was performed with DAPI (blue, scale bar $20 \mu \mathrm{m})$. L marks the arterial lumen. 


\section{Discussion}

In this study, we used JAM-A-targeted PBCA MB as contrast agent for molUS imaging in order to identify early inflammatory endothelial sites which could develop towards atherosclerotic areas. The luminal availability of JAM-A significantly increased on the endothelium after acute decrease in blood flow in the LCA due to PL. As a result of the flow alteration in the LCA and local inflammation, atherosclerotic plaque growth was induced, leading to progressive lumen narrowing. Moreover, atherosclerotic lesion formation was associated with an increase in vascular wall thickness and a decrease in vascular wall elasticity during systole and diastole, resembling the characteristics of atherosclerotic vessels in humans. The decreased perfusion as well as oscillatory flow ${ }^{3}$ represents a major local risk for the development of atherosclerotic lesions, which also induces a low-level inflammatory state ${ }^{25}$. The onset of plaque growth and endothelial activation was sensitively detected using MBJAM-A 2 weeks after vessel ligation. The inflammation was locally restricted as systemic inflammation markers did not change during the entire timeframe of the experiment.

Due to blood flow restriction through the PL carotid, a redistribution of blood flow through the RCA occurs, increasing the perfusion and by these means increasing the pressure and flow associated with a compensatory arterial wall thickness as an adaptation mechanism. It has already been described by Fry et al. ${ }^{26}$ that high wall shear stress has an athero-protective role, but that a sudden change in flow, determining oscillatory shear stress ${ }^{27}$ after $\mathrm{PL}$, is the promotor of atherosclerosis. This explains our findings in the RCA, where an acute increase in blood flow from normal state to a high state induces transitory endothelial dysfunction that was faithfully detected with MBJAM-A (2 weeks after PL) until the adaptation of the vessel was completed ( 3 weeks after PL) and high flow became protective. This hypothesis is further supported by the lack of elevated systemic inflammation markers that would suggest a systemic inflammatory reaction. Thus, we postulate that endothelial cells are able to react towards temporal shear stress fluctuations ${ }^{25,28}$, but also to adapt when the blood flow stabilizes ${ }^{28}$. In this context, 
JAM-A expression and imaging faithfully depict the biochemical changes taking place at the endothelial level, highlighting the potential of JAM-A as a versatile biochemical marker for endothelial activation.

In the WI-model of endothelial denudation, we confirmed JAM-A as a marker of endothelial activation and we were able to follow endothelial activation throughout the entire regeneration process using MBJAM-A-enhanced molUS. JAM-A was luminally exposed during endothelial regeneration starting on the first day postdenudation. However, arterial remodeling of the contralateral carotid and the adaptation of blood flow was not accompanied by a temporary endothelial JAMA translocation as in the PL-model. This can be explained by the fact that the stenosis of the LCA develops slowly in the WI-model and thus there is no acute change in pressure and oscillatory flow on the contralateral vessel, which would cause a temporarily enhance JAM-A expression.

Despite these promising results there is still optimization required for the molUS method. Although it is principally favorable that short circulation times and high flow conditions keep unspecific binding of MB to the glycocalyx and internalization by macrophages low ${ }^{29}$, it also limits the time span during which MB can bind to their target. Additionally, due to the fast blood flow in the arteries, the retention of $M B$ at the target is relatively short, which can make it difficult to investigate larger vessel areas by US. However, compared to our study in mice, a clinical setup would benefit from the larger vessel diameters, the higher vessel surface area, and the slower arterial flow. Moreover, by using smaller targeting moieties and by increasing the number of targeting ligands on the MB surface, e.g. by MB functionalization with dendrimeric spacers ${ }^{30}$, the target affinity and binding strength of MB could be improved.

Another limitation of US is its restriction to segmental body imaging, while PET, SPECT and MRI could be applied for whole body screening for predilection sites of atherosclerosis. Therefore, JAM-A-targeted probes for PET, SPECT and MRI may be generated. For this purpose, the same binding moiety may be used highlighting the broad scope of the presented molecular imaging approach. 
In conclusion, we demonstrate that non-invasive molUS with JAM-A-targeted MB is capable of detecting focal areas of transient endothelial activation triggered by the oscillatory blood flow condition. Thus, molUS with JAM-A-targeted MB may have the capacity to indicate predisposed areas of atherosclerotic plaque development, the onset of atherosclerosis and its normalization during therapy.

\section{Clinical competencies}

Acute blood flow fluctuations in healthy arteries induce transitory endothelial dysfunction leading to temporary JAM-A expression, which can be faithfully detected using molUS imaging employing JAM-A-targeted microbubbles. As JAM-A is suggested as a valuable imaging biomarker to assess early stages of atherosclerotic plaque formation, molUS of JAM-A may have the capacity to delineate predisposed areas of atherosclerotic plaque development, and the alternation in the onset and remission of atherosclerosis.

\section{Translational outlook}

In order to translate molUS of JAM-A to the clinic, the MB composition needs to be modified and the biotin-streptavidin-based conjugation of targeting ligands replaced to avoid immunological reactions in humans. Furthermore, for whole body screening approaches, JAM-A-targeted probes may be developed for other imaging modalities like PET and SPECT.

\section{Acknowledgments}

This Research was supported by the Deutsche Forschungsgemeinschaft (Forschergruppe FOR809-TP4/TP6/TP12, Sonderforschungsbereich 1123-A1), I3TM (SF_15_05_04), and IZKF Aachen (Junior Research Group to Dr. Liehn and K7-6/IA 531415 Prof. Kiessling/ Prof. van Zandvoort).

\section{Disclosures}

None. 


\section{References}

1. Liehn EA, Zernecke A, Postea O, Weber C. Chemokines: inflammatory mediators of atherosclerosis. Archives of physiology and biochemistry. 2006;112(4-5):229-238.

2. Kumar S, Kim CW, Simmons RD, Jo H. Role of flow-sensitive microRNAs in endothelial dysfunction and atherosclerosis: mechanosensitive athero-miRs. Arteriosclerosis, thrombosis, and vascular biology. 2014;34(10):2206-2216.

3. Peiffer V, Sherwin SJ, Weinberg PD. Does low and oscillatory wall shear stress correlate spatially with early atherosclerosis? A systematic review. Cardiovascular research. 2013;99(2):242-250.

4. Perez-Medina C, Binderup T, Lobatto ME, et al. In Vivo PET Imaging of HDL in Multiple Atherosclerosis Models. J Am Coll Cardiol Img. 2016;9(8):950961.

5. Danad I, Fayad ZA, Willemink MJ, Min JK. New Applications of Cardiac Computed Tomography: Dual-Energy, Spectral, and Molecular CT Imaging. J Am Coll Cardiol Img. 2015;8(6):710-723.

6. Pagoto A, Stefania R, Garello F, et al. Paramagnetic Phospholipid-Based Micelles Targeting VCAM-1 Receptors for MRI Visualization of Inflammation. Bioconjugate chemistry. 2016.

7. Kaufmann BA, Carr CL, Belcik JT, et al. Molecular imaging of the initial inflammatory response in atherosclerosis: implications for early detection of disease. Arteriosclerosis, thrombosis, and vascular biology. 2010;30(1):5459.

8. Curaj A, Wu Z, Fokong S, et al. Noninvasive molecular ultrasound monitoring of vessel healing after intravascular surgical procedures in a preclinical setup. Arteriosclerosis, thrombosis, and vascular biology. 2015;35(6):13661373.

9. Abou-Elkacem L, Bachawal SV, Willmann JK. Ultrasound molecular imaging: Moving toward clinical translation. Eur J Radiol. 2015;84(9):1685-1693.

10. Rix A, Fokong $S$, Heringer $S$, et al. Molecular Ultrasound Imaging of alphavbeta3-Integrin Expression in Carotid Arteries of Pigs After Vessel Injury. Investigative radiology. 2016. 
11. Wu Z, Curaj A, Fokong $\mathrm{S}$, et al. Rhodamine-loaded intercellular adhesion molecule-1-targeted microbubbles for dual-modality imaging under controlled shear stresses. Circulation. Cardiovascular imaging. 2013;6(6):974-981.

12. Nahrendorf $\mathrm{M}$, Keliher $\mathrm{E}$, Panizzi $\mathrm{P}$, et al. 18F-4V for PET-CT imaging of VCAM-1 expression in atherosclerosis. J Am Coll Cardiol Img. 2009;2(10):1213-1222.

13. Daeichin V, Kooiman K, Skachkov I, et al. Quantification of Endothelial alphavbeta3 Expression with High-Frequency Ultrasound and Targeted Microbubbles: In Vitro and In Vivo Studies. Ultrasound in medicine \& biology. 2016.

14. Davies MJ, Gordon JL, Gearing AJ, et al. The expression of the adhesion molecules ICAM-1, VCAM-1, PECAM, and E-selectin in human atherosclerosis. J Pathol. 1993;171(3):223-229.

15. Lindner V, Collins T. Expression of NF-kappa B and I kappa B-alpha by aortic endothelium in an arterial injury model. Am J Pathol. 1996;148(2):427-438.

16. Zeiffer U, Schober A, Lietz M, et al. Neointimal smooth muscle cells display a proinflammatory phenotype resulting in increased leukocyte recruitment mediated by P-selectin and chemokines. Circ Res. 2004;94(6):776-784.

17. Videm V, Albrigtsen M. Soluble ICAM-1 and VCAM-1 as markers of endothelial activation. Scandinavian journal of immunology. 2008;67(5):523531.

18. Zhang YJ, Bai DN, Du JX, et al. Ultrasound-guided imaging of junctional adhesion molecule-A-targeted microbubbles identifies vulnerable plaque in rabbits. Biomaterials. 2016;94:20-30.

19. Schmitt MM, Megens RT, Zernecke A, et al. Endothelial junctional adhesion molecule-a guides monocytes into flow-dependent predilection sites of atherosclerosis. Circulation. 2014;129(1):66-76.

20. Ostermann G, Weber KS, Zernecke A, Schroder A, Weber C. JAM-1 is a ligand of the beta(2) integrin LFA-1 involved in transendothelial migration of leukocytes. Nature immunology. 2002;3(2):151-158.

21. Bazzoni G. The JAM family of junctional adhesion molecules. Current opinion in cell biology. 2003;15(5):525-530. 
22. Schmitt MM, Fraemohs L, Hackeng TM, Weber C, Koenen RR. Atherogenic mononuclear cell recruitment is facilitated by oxidized lipoprotein-induced endothelial junctional adhesion molecule-A redistribution. Atherosclerosis. 2014;234(2):254-264.

23. Fokong S, Siepmann M, Liu Z, Schmitz G, Kiessling F, Gatjens J. Advanced characterization and refinement of poly N-butyl cyanoacrylate microbubbles for ultrasound imaging. Ultrasound in medicine \& biology. 2011;37(10):16221634.

24. Kiessling F, Bzyl J, Fokong S, Siepmann M, Schmitz G, Palmowski M. Targeted ultrasound imaging of cancer: an emerging technology on its way to clinics. Curr Pharm Des. 2012;18(15):2184-2199.

25. Davies PF, Civelek M, Fang Y, Fleming I. The atherosusceptible endothelium: endothelial phenotypes in complex haemodynamic shear stress regions in vivo. Cardiovascular research. 2013;99(2):315-327.

26. Caro CG, Fitz-Gerald JM, Schroter RC. Atheroma and arterial wall shear. Observation, correlation and proposal of a shear dependent mass transfer mechanism for atherogenesis. Proceedings of the Royal Society of London. Series B, Biological sciences. 1971;177(1046):109-159.

27. Ku DN, Giddens DP, Zarins CK, Glagov S. Pulsatile flow and atherosclerosis in the human carotid bifurcation. Positive correlation between plaque location and low oscillating shear stress. Arteriosclerosis. 1985;5(3):293-302.

28. Davies PF, Dewey CF, Jr., Bussolari SR, Gordon EJ, Gimbrone MA, Jr. Influence of hemodynamic forces on vascular endothelial function. In vitro studies of shear stress and pinocytosis in bovine aortic cells. The Journal of clinical investigation. 1984;73(4):1121-1129.

29. Bioley G, Bussat P, Lassus A, Schneider M, Terrettaz J, Corthesy B. The phagocytosis of gas-filled microbubbles by human and murine antigenpresenting cells. Biomaterials. 2012;33(1):333-342.

30. Barge A, Caporaso M, Cravotto G, et al. Design and Synthesis of a gamma(1)beta(8)-Cyclodextrin Oligomer: A New Platform with Potential Application as a Dendrimeric Multicarrier. Chemistry. 2013;19(36):1208612092. 
Molecular ultrasound imaging of JAM-A 



\section{Chapter 6}

General discussion 


\section{Murine models for studying atherosclerosis}

Atherosclerosis is the main pathology underlying cardiovascular diseases, therefore it is of great interest to understand the etiology, pathophysiology, and complications of the disease in order to improve current treatment and to develop new therapeutic strategies. Since studies on human subjects have obvious ethical and experimental limitations, a variety of animal models, such as rabbits ${ }^{1,2}$, rats $^{3}$, mice $^{4}$, swine ${ }^{5}$, guinea pigs ${ }^{6}$, dogs $^{7}$, and non-human primates ${ }^{8}$, have been used for investigating the mechanisms involved in the induction, progression, and regression of atherosclerotic lesions. An ideal animal model for studying human disease, in particular atherosclerosis, should possess several features including: 1) the close resemblance to human conditions, 2) the capability to develop various stages of the disease, including fatty streaks, vulnerable and stable plaques, as well as 3) the presence of relevant complications such as calcification, ulceration, hemorrhage, plaque rapture, thrombosis, stenosis, neointima formation, and aneurysms. Even though animal models that replicate human atherosclerosis have been generated, each of current animal models has various limitations ${ }^{9}$ and there is no unanimity concerning the ideal animal model.

The first mouse model for induced atherosclerosis was developed by Wissler and coworkers in $1960 \mathrm{~s}^{10}$, and since then, murine atherosclerosis has been intensively investigated. Initial limitations of the mouse model, such as their natural resistance to the atherosclerotic disease due to a lower plasma cholesterol levels of 60-100 mg/dl compared to humans, were quickly removed with the introduction of transgenic hyperlipidemic animals which develop atherosclerotic lesions. The high genetic similarities to humans, variations and combinations of transgenic models, ease of genetic manipulation, and the relatively short time frame for the development of atherosclerosis, make mice the currently most extensively used model in cardiovascular research. The small size of mice also has an advantage in that only low amounts of antibodies and drugs are needed for interventions and imaging, which is financially important. Moreover, there are several other advantages such as the relative low-cost to 
purchase, breed, feed and maintain, ease to handle, and controllable diet and environmental risk factors, which make them the leaders in experimental use.

\section{Atherosclerotic disease of the carotid artery}

Carotid arteries have been intensively used for studying biological processes involved in atherosclerosis ${ }^{11-16}$, but they have not been into focus of imaging protocols. Carotids are particularly of high interest to be studied due to the existence of experimental models for developing both native atherosclerosis, and neointima following revascularization procedures.

Particularly, in the present studies carotid arteries have been chosen to be investigated for several reasons. Firstly, carotid arteries are superficial arteries and therefore are easily accessible to TPLSM for in vivo analysis. Secondly, carotid US imaging is routinely applied in the clinical practice and therefore the imaging protocols could be more easily translated from bench-side to clinical use. Moreover, murine carotids are very attractive due to their size which is even smaller than the human coronary arteries ${ }^{17}$. Therefore, molecular imaging protocols applied for murine carotid arteries could be translated for human coronary arteries investigation.

\section{Advances in molecular imaging of arteries subjected to high wall shear stress}

Imaging has become an indispensable tool both in cardiovascular research and clinical practice. Various imaging technologies which are now available as clinical diagnostic tools, are mostly restricted to morphological and functional imaging to illustrate the anatomy and quantify the degree of vessel obstruction. However, the anatomical severity of stenosis does not sufficiently determine the risk of vascular events. Therefore, multiple approaches with focus on molecular imaging are been pursued, aiming specifically at the detection and characterization of various biological conditions. Imaging modalities including US, PET, SPECT, 
MRI, CT, and optical imaging, such as TPLSM, have been used for imaging various animal models of atherosclerosis, primarily $\mathrm{ApoE}^{-/-}$and $\mathrm{LDLr}^{-/-}$mice and hyperlipidemic rabbits ${ }^{18}$. So far, studies have concentrated on the atherosclerotic plaque composition and the detection of vulnerable plaques, and these protocols are slowly being translated into clinical application ${ }^{18,19}$.

However, there are some fundamental technical aspects which keep many of these imaging modalities away from clinical implementation. Optical imaging technologies are not feasible for human whole-body imaging, as it is for small laboratory animals, because light does not propagate deeply into skin and tissues. PET as a routine clinical diagnostic tool has been impeded by the need for an on-site cyclotron and a radiochemistry unit for the production of the shortlived radiotracer ${ }^{20}$. Moreover, the main limitation to $18 \mathrm{~F}$-fluorodeoxyglucose (18FFDG) PET imaging of the coronary arteries is myocardial uptake ${ }^{21}$. Alternatively, molecular US imaging is the most accessible imaging modality to be translated into clinical diagnostics, due to the already trained medical personnel, broad availability of the device, and contrast tracers, which have already been included in the clinical practice 22,23 . However, molecular US imaging protocols need to be first validated with sensitive methods which can analyze tissues at molecular level. Optical imaging modalities such as TPLSM offer high spatial resolution and proper tissue penetration and can visualize target availability on cell surface as well single targeted MB coupled with its endothelial receptor. Therefore, TPLSM can be used as an imaging tool to overcome the sensitivity issue of US imaging, validating imaging protocols before their clinical translation. Hence, the two imaging modalities have been employed as main investigation tools in the studies included in Chapter 2 and Chapter 4.

In vivo molecular imaging of carotid atherosclerosis is challenging as the vessels move rapidly with heart beat and respiration, which can cause image distortions and artifacts. Additionally, in big arteries such as the aorta, carotid, renal, and iliac arteries, the wall shear stress is high as the blood flow is generally laminar. Only at the vessel curvatures and bifurcations, the flow becomes turbulent and shear stress is low. As the high wall shear stress makes molecular imaging of 
endothelial markers difficult, inflammation in arteries subjected to high wall shear stress ${ }^{24-26}$ was intensively investigated in certain areas with decreased shear stress such as the aortic root ${ }^{26,27}$ and $\operatorname{arch}^{28}$, which are as well relative large areas to be examined. Even though, very attractive as models for atherosclerotic pathology, with high clinical implications, carotid arteries have not been in the focus of imaging protocols. Molecular imaging of endothelial dysfunction in the common carotid artery, where the shear stress is high, is very challenging. In such areas, the bond between endothelial receptors and molecular tracers should be able to resist the shear stress and mechanical forces applied by circulating blood cells.

This thesis offers a structured approach on the molecular TPLSM/US carotid artery imaging of luminally exposed endothelial markers which upregulate with endothelial dysfunction during different stages of atherosclerotic disease. First, targeted MB specific retention under high shear and flow conditions in the mouse carotid artery, and their capacity to resist the shear stress and mechanical forces applied by circulating blood cells was evaluated with TPLSM and US imaging by employing two molecular targets, namely ICAM-1 (Chapter 2) and VCAM-1 (Chapter 4). For this purpose, the ex vivo binding studies have been performed by injecting the MB suspension in whole blood under anticoagulatory conditions at $37^{\circ} \mathrm{C}$. Hence, we show MB binding capacity at physiological shear stress conditions of 35-60 dyn $/ \mathrm{cm}^{2}$.

Next, molecular TPLSM/US imaging was applied to evaluate specific atherosclerotic lesions developed in the carotid arteries, considering two highimpact clinical situations: revascularization procedures (Chapter 4) and patients with hypercholesterolemia and oscillatory arterial blood pressure (Chapter 5). Therefore, two animal models, the $\mathrm{WI}$ and the $\mathrm{PL}$, mimicking these clinically relevant situations were investigated, by employing molecular US (Chapter 4 and Chapter 5) and TPLSM imaging (Chapter 4).

The challenges of my current studies were to find high-potential matches between the ligand-receptor and the mimicking clinical situation, the neointima formation (Chapter 4) or flow induced endothelial activation (Chapter 5). Moreover, given 
the case of endothelial denudation followed by neointima formation (Chapter 4), the target for the molecular tracer should fulfil several criteria: 1) subendothelial expression after endothelial denudation (e.g. expression by luminally exposed vascular SMC); 2) expression by the activated regenerating $E C$; and 3 ) absence/low constitutive expression after reendothelialization. Several wellknown atherosclerotic markers such as PECAM-1, ICAM-1, VCAM-1, and P- and E-Selectin have been considered. From those, VCAM-1 was chosen due to several reasons: 1) VCAM-1 is one of the most well-known atherosclerotic markers $\left.{ }^{29} ; 2\right)$ it is an acute inflammatory marker that is highly expressed after vascular injury, and it plays a major role in the formation of neointima and restenosis $^{30}$; 3) more importantly, VCAM-1 was previously described to be expressed by both $\mathrm{EC}^{31}$ and vascular $\mathrm{SMC}^{32,33}$ in atherosclerotic arteries. Additionally, the other markers (PECAM-1, ICAM-1, P- and E-Selectin) were excluded, since they are known to have a constitutive expression level ${ }^{34-38}$ while some of them might either be not sufficiently expressed on activated vascular SMC (PECAM-1, E-Selectin, P-Selectin) ${ }^{37,39,40}$ or remain to be upregulated for a prolonged period of time after complete endothelial structural recovery.

Further, in Chapter 5, targeted MB binding capacity, which was proven in Chapter 2 and Chapter 4, was applied for advancing an imaging protocol in the second clinically-related situation, the transient endothelial activation and dysfunctionality triggered by the oscillatory blood flow condition accompanied by hyperlipidemia. JAM-A is the biological marker most responsive to acute changes in blood flow ${ }^{41}$, being up-regulated specifically on EC at the flow-dependent predilection sites of atherosclerosis ${ }^{42}$. Therefore, JAM-A was chosen as biological marker associated to flow-induced endothelial dysfunction and employed in an US imaging protocol for monitoring endothelial reactivity to blood flow disturbances. In this study, we show the sensitive reaction of JAM-A upregulation and translocation to the endothelial surface following both a sudden decrease (in the PL carotid) and increase (in the contralateral carotid) of blood flow in the carotid arteries, and the capacity of molecular US imaging to detect and monitor this biological process. Thus, molecular US with JAM-A targeted MB may have the capacity to indicate predisposed areas of atherosclerotic plaque 
development in big arteries, the onset of atherosclerosis, and its normalization during therapy.

Although we refer to carotids as big arteries, the diameter of a mouse carotid artery is approximately $0.3-0.5 \mathrm{~mm}$, while the length that is accessible for in vivo imaging with tools such as TPLSM and US is limited to approximately 9-12 $\mathrm{mm}$. The overall small size of the artery, the high heart rate (300-500 bpm), and the fast respiration movements make imaging particularly difficult and challenging. However, these obstacles have been managed by 1 ) increasing the group size included in the study to obtain statistically relevant results, 2) usage of resonant scanning ultrafast intravital TPLSM, 3) training in handling the imaging devices, and 4) by finding biological targets best suitable for the purpose of each study. Hence, with this work, the horizon of molecular US applicability has been enlarged from imaging primary tumors ${ }^{43-47}$, metastasis ${ }^{43}$ or carcinoma xenografts $^{48,49}$, drug delivery ${ }^{50,51}$, myocardial ischemia in mice ${ }^{52}$, skeletal muscle vascularization $^{53-55}$, and vascular endothelium of areas subjected to low shear stress in small and big arteries ${ }^{28,56}$ to molecular imaging of vascular endothelium in arteries subjected to high shear stress. Furthermore, the use of targeted MB in combination with TPLSM offers new perspectives on microscopy imaging, in addition to the routine use of TPLSM for imaging liver ${ }^{57}$, bladder ${ }^{58-60}$ and ureter ${ }^{61}$ tissues, lung fibrosis ${ }^{62}$, and collagen activity in wounded skin $^{63}$.

\section{Future perspectives}

In the current studies, two high-impact clinical situations: 1) revascularization procedures (Chapter 3 and Chapter $\mathbf{4}$ ) and 2) patients with hypercholesterolemia and transitory oscillatory arterial blood pressure (Chapter 5) have been addressed from the concept to the application point of view. However, one further aspect with high clinical significance on early diagnosis should be investigated next: molecular imaging of in-stent neointima formation. In-stent molecular US imaging would have a great impact on the clinical practice. So far, the limitation of this approach is the lack of echogenic compatible stents for the mouse model. 


\section{Chapter 6}

Therefore, the development of small size echogenic stents would be of high interest and would offer new possibilities for non-invasive long-term monitoring of patient recovery.

In addition, clinical implementation of molecular US by employing ICAM-1, VCAM-1, or JAM-A-targeted MB requires MB modification to avoid the currently used biotin-streptavidin-based conjugation of targeting ligands in order to prevent immunological reactions in humans.

Furthermore, for whole body screening approaches, JAM-A targeted probes may be developed for other imaging modalities like PET and SPECT. 


\section{References}

1. Aliev G, Burnstock G. Watanabe rabbits with heritable hypercholesterolaemia: a model of atherosclerosis. Histol Histopathol. 1998;13(3):797-817.

2. Brousseau ME, Hoeg JM. Transgenic rabbits as models for atherosclerosis research. J Lipid Res. 1999;40(3):365-375.

3. Joris I, Zand T, Nunnari JJ, Krolikowski FJ, Majno G. Studies on the pathogenesis of atherosclerosis. I. Adhesion and emigration of mononuclear cells in the aorta of hypercholesterolemic rats. Am $\mathrm{J}$ Pathol. 1983;113(3):341-358.

4. Stewart-Phillips JL, Lough J. Pathology of atherosclerosis in cholesterol-fed, susceptible mice. Atherosclerosis. 1991;90(2-3):211-218.

5. Luginbuhl H, Pauli B, Ratcliffe HL. Atherosclerosis in swine and swine as a model for the study of atherosclerosis. Adv Cardiol. 1974;13:119-126.

6. Fernandez ML, Volek JS. Guinea pigs: a suitable animal model to study lipoprotein metabolism, atherosclerosis and inflammation. Nutr Metab (Lond). 2006;3:17.

7. Mahley RW, Weisgraber $\mathrm{KH}$, Innerarity T. Canine lipoproteins and atherosclerosis. II. Characterization of the plasma lipoproteins associated with atherogenic and nonatherogenic hyperlipidemia. Circ Res. 1974;35(5):722-733.

8. Bullock BC, Lehner ND, Clarkson TB, Feldner MA, Wagner WD, Lofland HB. Comparative primate atherosclerosis. I. Tissue cholesterol concentration and pathologic anatomy. Exp Mol Pathol. 1975;22(2):151-175.

9. Kapourchali FR, Surendiran G, Chen L, Uitz E, Bahadori B, Moghadasian $\mathrm{MH}$. Animal models of atherosclerosis. World J Clin Cases. 2014;2(5):126132.

10. Vesselinovitch D, Wissler RW, Doull J. Experimental production of atherosclerosis in mice. 1. Effect of various synthetic diets and radiation on survival time, food consumption and body weight in mice. J Atheroscler Res. 1968;8(3):483-495. 
11. Simsekyilmaz S, Schreiber F, Weinandy S, Gremse F, Sonmez TT, Liehn EA. A murine model of stent implantation in the carotid artery for the study of restenosis. J Vis Exp. 2013(75):e50233.

12. Simsekyilmaz S, Liehn EA, Weinandy S, et al. Targeting In-Stent-Stenosis with RGD- and CXCL1-Coated Mini-Stents in Mice. PLoS One. 2016;11(5):e0155829.

13. Noels H, Zhou B, Tilstam PV, et al. Deficiency of endothelial CXCR4 reduces reendothelialization and enhances neointimal hyperplasia after vascular injury in atherosclerosis-prone mice. Arterioscler Thromb Vasc Biol. 2014;34(6):1209-1220.

14. Simsekyilmaz S, Cabrera-Fuentes HA, Meiler S, et al. Role of extracellular RNA in atherosclerotic plaque formation in mice. Circulation. 2014;129(5):598-606.

15. Liehn EA, Piccinini AM, Koenen RR, et al. A new monocyte chemotactic protein-1/chemokine CC motif ligand-2 competitor limiting neointima formation and myocardial ischemia/reperfusion injury in mice. J Am Coll Cardiol. 2010;56(22):1847-1857.

16. Zernecke A, Liehn EA, Fraemohs L, et al. Importance of junctional adhesion molecule-A for neointimal lesion formation and infiltration in atherosclerosisprone mice. Arterioscler Thromb Vasc Biol. 2006;26(2):e10-13.

17. Leung WH, Stadius ML, Alderman EL. Determinants of normal coronary artery dimensions in humans. Circulation. 1991;84(6):2294-2306.

18. Leuschner F, Nahrendorf M. Molecular imaging of coronary atherosclerosis and myocardial infarction: considerations for the bench and perspectives for the clinic. Circ Res. 2011;108(5):593-606.

19. Quillard T, Libby P. Molecular imaging of atherosclerosis for improving diagnostic and therapeutic development. Circ Res. 2012;111(2):231-244.

20. Pichler BJ, Wehrl HF, Judenhofer MS. Latest advances in molecular imaging instrumentation. J Nucl Med. 2008;49 Suppl 2:5S-23S.

21. Rosenbaum D, Millon A, Fayad ZA. Molecular imaging in atherosclerosis: FDG PET. Curr Atheroscler Rep. 2012;14(5):429-437.

22. Imai K, Kotani T, Tsuda H, Nakano T, Hirakawa A, Kikkawa F. A Novel Approach to Detecting Postpartum Hemorrhage Using Contrast-Enhanced Ultrasound. Ultrasound Med Biol. 2016. 
23. Tan Y, Xie X, Lin Y, Huang T, Huang G. Hepatic epithelioid angiomyolipoma: clinical features and imaging findings of contrast-enhanced ultrasound and CT. Clin Radiol. 2016.

24. Dinkelborg LM, Duda SH, Hanke H, Tepe G, Hilger CS, Semmler W. Molecular imaging of atherosclerosis using a technetium-99m-labeled endothelin derivative. J Nucl Med. 1998;39(10):1819-1822.

25. Winter PM, Morawski AM, Caruthers SD, et al. Molecular imaging of angiogenesis in early-stage atherosclerosis with alpha(v)beta3-integrintargeted nanoparticles. Circulation. 2003;108(18):2270-2274.

26. Nahrendorf M, Jaffer FA, Kelly KA, et al. Noninvasive vascular cell adhesion molecule-1 imaging identifies inflammatory activation of cells in atherosclerosis. Circulation. 2006;114(14):1504-1511.

27. Nahrendorf $\mathrm{M}$, Keliher E, Panizzi $\mathrm{P}$, et al. 18F-4V for PET-CT imaging of VCAM-1 expression in atherosclerosis. JACC Cardiovasc Imaging. 2009;2(10):1213-1222.

28. Kaufmann BA, Sanders JM, Davis C, et al. Molecular imaging of inflammation in atherosclerosis with targeted ultrasound detection of vascular cell adhesion molecule-1. Circulation. 2007;116(3):276-284.

29. Cybulsky MI, liyama K, Li H, et al. A major role for VCAM-1, but not ICAM-1, in early atherosclerosis. J Clin Invest. 2001;107(10):1255-1262.

30. Qu $\mathrm{Y}$, Shi $\mathrm{X}$, Zhang $\mathrm{H}$, et al. VCAM-1 siRNA reduces neointimal formation after surgical mechanical injury of the rat carotid artery. J Vasc Surg. 2009;50(6):1452-1458.

31. Nakashima Y, Raines EW, Plump AS, Breslow JL, Ross R. Upregulation of VCAM-1 and ICAM-1 at atherosclerosis-prone sites on the endothelium in the ApoE-deficient mouse. Arterioscler Thromb Vasc Biol. 1998;18(5):842851.

32. Bobryshev YV, Lord RS, Rainer SP, Munro VF. VCAM-1 expression and network of VCAM-1 positive vascular dendritic cells in advanced atherosclerotic lesions of carotid arteries and aortas. Acta Histochem. 1996;98(2):185-194.

33. Hastings NE, Feaver RE, Lee MY, Wamhoff BR, Blackman BR. Human IL-8 regulates smooth muscle cell VCAM-1 expression in response to endothelial 
cells exposed to atheroprone flow. Arterioscler Thromb Vasc Biol. 2009;29(5):725-731.

34. Lindner V, Collins T. Expression of NF-kappa B and I kappa B-alpha by aortic endothelium in an arterial injury model. Am J Pathol. 1996;148(2):427-438.

35. Wu Z, Curaj A, Fokong S, et al. Rhodamine-loaded intercellular adhesion molecule-1-targeted microbubbles for dual-modality imaging under controlled shear stresses. Circ Cardiovasc Imaging. 2013;6(6):974-981.

36. Davies MJ, Gordon JL, Gearing AJ, et al. The expression of the adhesion molecules ICAM-1, VCAM-1, PECAM, and E-selectin in human atherosclerosis. J Pathol. 1993;171(3):223-229.

37. Ilan N, Madri JA. PECAM-1: old friend, new partners. Curr Opin Cell Biol. 2003;15(5):515-524.

38. Zeiffer U, Schober A, Lietz M, et al. Neointimal smooth muscle cells display a proinflammatory phenotype resulting in increased leukocyte recruitment mediated by P-selectin and chemokines. Circ Res. 2004;94(6):776-784.

39. Schober A, Zernecke A, Liehn EA, et al. Crucial role of the CCL2/CCR2 axis in neointimal hyperplasia after arterial injury in hyperlipidemic mice involves early monocyte recruitment and CCL2 presentation on platelets. Circ Res. 2004;95(11):1125-1133.

40. Kennedy S, McPhaden AR, Wadsworth RM, Wainwright CL. Correlation of leukocyte adhesiveness, adhesion molecule expression and leukocyteinduced contraction following balloon angioplasty. $\mathrm{Br} J$ Pharmacol. 2000;130(1):95-103.

41. Schmitt MM, Megens RT, Zernecke A, et al. Endothelial junctional adhesion molecule-a guides monocytes into flow-dependent predilection sites of atherosclerosis. Circulation. 2014;129(1):66-76.

42. Ostermann G, Weber KS, Zernecke A, Schroder A, Weber C. JAM-1 is a ligand of the beta(2) integrin LFA-1 involved in transendothelial migration of leukocytes. Nat Immunol. 2002;3(2):151-158.

43. Bartolotta TV, Taibbi A, Picone D, Anastasi A, Midiri M, Lagalla R. Detection of liver metastases in cancer patients with geographic fatty infiltration of the liver: the added value of contrast-enhanced sonography. Ultrasonography. 2016. 
44. Tzou DT, Weinstein S, Usawachintachit M, Mongan J, Greene KL, Chi T. Contrast Enhanced Ultrasound Detects Recurrent Renal Cell Carcinoma in the Setting of Chronic Renal Insufficiency. Clin Genitourin Cancer. 2016.

45. van Sloun R, Demi L, Postema A, de la Rosette J, Wijkstra H, Mischi M. Entropy of Ultrasound-Contrast-Agent Velocity Fields for Angiogenesis Imaging in Prostate Cancer. IEEE Trans Med Imaging. 2016.

46. Schalk S, Demi L, Bouhouch N, et al. Contrast-enhanced Ultrasound Angiogenesis Imaging by Mutual Information Analysis for Prostate Cancer Localization. IEEE Trans Biomed Eng. 2016.

47. Bzyl J, Palmowski M, Rix A, et al. The high angiogenic activity in very early breast cancer enables reliable imaging with VEGFR2-targeted microbubbles (BR55). Eur Radiol. 2013;23(2):468-475.

48. Spivak I, Rix A, Schmitz G, et al. Low-Dose Molecular Ultrasound Imaging with E-Selectin-Targeted PBCA Microbubbles. Mol Imaging Biol. 2016;18(2):180-190.

49. Baetke SC, Rix A, Tranquart F, et al. Squamous Cell Carcinoma Xenografts: Use of VEGFR2-targeted Microbubbles for Combined Functional and Molecular US to Monitor Antiangiogenic Therapy Effects. Radiology. 2016;278(2):430-440.

50. Dasgupta A, Liu M, Ojha T, Storm G, Kiessling F, Lammers T. Ultrasoundmediated drug delivery to the brain: principles, progress and prospects. Drug Discov Today Technol. 2016;20:41-48.

51. Unger E, Porter T, Lindner J, Grayburn P. Cardiovascular drug delivery with ultrasound and microbubbles. Adv Drug Deliv Rev. 2014;72:110-126.

52. Mott B, Packwood W, Xie A, et al. Echocardiographic Ischemic Memory Imaging Through Complement-Mediated Vascular Adhesion of Phosphatidylserine-Containing Microbubbles. JACC Cardiovasc Imaging. 2016;9(8):937-946.

53. Wu MD, Belcik JT, Qi Y, et al. Abnormal Regulation of Microvascular Tone in a Murine Model of Sickle Cell Disease Assessed by Contrast Ultrasound. J Am Soc Echocardiogr. 2015;28(9):1122-1128.

54. Belcik JT, Davidson BP, Foster T, et al. Contrast-enhanced ultrasound assessment of impaired adipose tissue and muscle perfusion in insulinresistant mice. Circ Cardiovasc Imaging. 2015;8(4). 
55. Seol SH, Davidson BP, Belcik JT, et al. Real-time contrast ultrasound muscle perfusion imaging with intermediate-power imaging coupled with acoustically durable microbubbles. J Am Soc Echocardiogr. 2015;28(6):718-726 e712.

56. Davidson BP, Chadderdon SM, Belcik JT, Gupta S, Lindner JR. Ischemic memory imaging in nonhuman primates with echocardiographic molecular imaging of selectin expression. J Am Soc Echocardiogr. 2014;27(7):786-793 e782.

57. Heymann F, Niemietz PM, Peusquens J, et al. Long term intravital multiphoton microscopy imaging of immune cells in healthy and diseased liver using CXCR6.Gfp reporter mice. J Vis Exp. 2015(97).

58. Schueth A, van Zandvoort MA, Buurman WA, van Koeveringe GA. Murine bladder imaging by 2-photon microscopy: an experimental study of morphology. J Urol. 2014;192(3):973-980.

59. Schueth A, Spronck B, van Zandvoort MA, van Koeveringe GA. Age-related changes in murine bladder structure and sensory innervation: a multiphoton microscopy quantitative analysis. Age (Dordr). 2016;38(1):17.

60. Klinger A, Orzekowsky-Schroeder R, von Smolinski D, et al. Complex morphology and functional dynamics of vital murine intestinal mucosa revealed by autofluorescence 2-photon microscopy. Histochem Cell Biol. 2012;137(3):269-278.

61. Spronck B, Merken JJ, Reesink KD, Kroon W, Delhaas T. Ureter smooth muscle cell orientation in rat is predominantly longitudinal. PLoS One. 2014;9(1):e86207.

62. Pena AM, Fabre A, Debarre D, et al. Three-dimensional investigation and scoring of extracellular matrix remodeling during lung fibrosis using multiphoton microscopy. Microsc Res Tech. 2007;70(2):162-170.

63. Torkian BA, Yeh AT, Engel R, Sun CH, Tromberg BJ, Wong BJ. Modeling aberrant wound healing using tissue-engineered skin constructs and multiphoton microscopy. Arch Facial Plast Surg. 2004;6(3):180-187. 
General discussion 



\section{Summary}

The endothelium is the inner lining of blood vessel walls with direct contact to the blood circulation. It plays a major role in all stages of atherosclerosis, from its initiation to plaque rupture and thrombosis. Endothelial dysfunction, triggered by abnormal shear stress, local inflammation, and chemokine release is followed by i) the upregulation of surface adhesion molecules, such as intercellular adhesion molecule (ICAM)-1, vascular cell adhesion molecule (VCAM)-1, and selectins, and ii) the translocation of platelet endothelial cell adhesion molecule (PECAM)-1 and junctional adhesion molecule (JAM)-A, which facilitate the transmigration of inflammatory cells from circulation into the arterial wall.

Oscillatory arterial blood pressure and hyperlipidemia, or injured endothelium following revascularization procedures predispose patients to develop atherosclerotic lesions and neointima, respectively. During revascularization procedures (Chapter 3 and Chapter $\mathbf{4}$ ), such as balloon angioplasty with/without stent implantation or endarterectomy, the endothelial layer is severely injured and partially or fully removed. Throughout the process of endothelial regeneration, the blood vessel is vulnerable to thrombus formation and restenosis. Recovering endothelial cells expose biomarkers on the luminal surface, which can be used as potential target for imaging and early diagnosis. Similarly, oscillatory arterial blood pressure and hyperlipidemia cause endothelial activation with luminal exposure of biological markers (Chapter 5).

In this study, the main aim was to establish a disease-specific molecular imaging vascular contrast agent that can withstand the physiological flow and shear stress conditions in major arteries. Therefore, two clinically related murine models of endothelial dysfunction mimicking endothelial denudation (Chapter 3 and Chapter 4) or oscillatory arterial blood pressure (Chapter 5) were used: the wireinjury $(\mathrm{WI})$ and the partial-ligation $(\mathrm{PL})$, respectively. The arteries were screened by immunohistology for particular biological markers. As contrast agent, polymerbased air-filled microbubbles (MB) with the diameter of 1-2 $\mu \mathrm{m}$ were employed. 
MB functionalization was performed by conjugation of specific antibodies to the MB surface. Fluorescent loading of the MB shell enabled the bimodal detection of the particles using both two-photon laser scanning microscopy (TPLSM) and molecular ultrasound (US). Clinical imaging modalities, such as US imaging, lack resolution for studying the interaction of microbubbles with the vascular wall. Therefore, TPLSM was applied as a deep-tissue imaging modality for the characterization of surface marker expression and binding kinetics of rhodamineloaded MB as a preclinical validation step.

Firstly, using TPLSM imaging on TNF- $\alpha$ stimulated explanted murine carotid arteries in an ex vivo flow chamber system, the shear stress resistance of ICAM-1-targeted MB (MBІСАM-1) was characterized at shear rates matching and exceeding physiological parameters (Chapter 2). ICAM-1 was chosen as target for endothelial inflammation after immunohistological screening of TNF- $\alpha$-stimulated carotids for several biological markers. TPLSM results were further used as validation of ex vivo and in vivo molecular US imaging employing MBICAM-1 in the same experimental animal model.

Next (Chapter 4), the endothelial recovery after arterial denudation was monitored by employing VCAM-1-targeted MB (MBVCAM-1). VCAM-1 is expressed immediately after endothelial denudation on the medial smooth muscle cells. During early vascular recovery, it is expressed on both smooth muscle cells and regenerating endothelium, but it disappears from the endothelial layer after its structural recovery. This makes it the perfect molecular marker to track endothelial healing. Using in vivo TPLSM, single MB bound to the vascular lumen were imaged and quantified showing specific retention. Translation into molecular US confirmed injury-specific MB retention and accurate assessment of the endothelial regeneration state in correlation with immunohistology and TPLSM imaging of the luminal surface.

Lastly, transient endothelial activation under acute blood flow variations was investigated in vivo by employing JAM-A-targeted MB (MBJAM-A) for molecular US imaging of carotid arteries subjected to PL (Chapter 5). JAM-A is an interendothelial marker which redistributes to the luminal surface upon inflammatory 
stimuli and endothelial activation. MBJAM-A bound specifically to JAM-A on activated endothelium and are able to identify the location of areas with endothelial dysfunction upon acute changes in blood flow. Our data indicate that not only flow reduction due to vessel obstruction in the partially ligated artery, but also acute flow increase due to compensatory blood flow redistribution in the contralateral carotid, induce luminal exposure of JAM-A. This illustrates the high sensitivity of this marker for assessing acute vascular vulnerability and remodeling.

In conclusion, this thesis provides new insights into molecular imaging of atherosclerosis and clinically relevant associated biological processes, specifically neointima formation and flow-induced endothelial activation. After further refinements, these imaging methods may become important tools in both the scientific and the clinical environment. 



\section{Valorization addendum}

\section{Relevance for society}

Cardiovascular disease is the leading cause of morbidity and mortality in the modern society ${ }^{1}$. Patients with clinical manifestation of atherosclerosis are regularly subjected to revascularization procedures such as balloon angioplasty with or without stent implantation or endarterectomy. Post-procedure, patients receive long term dual antiplatelet therapy to ensure thrombosis prophylaxis until full endothelial recovery. Meanwhile, secondary interventional procedures with high risk of bleeding, such as dental extractions or surgical interventions in multimorbid patients are denied. It is of high clinical relevance to develop a screening protocol to monitor early endothelial recovery which could help in personalization of medication and deciding the cessation of otherwise unnecessarily prolonged medication.

Additionally, cardiovascular patients with subclinical atherosclerosis usually have accompanying pathologies such as oscillatory arterial blood pressure. Our results suggest that molecular ultrasound imaging of acute endothelial activation may be suitable to assess the risk of patients with oscillatory arterial blood pressure to develop biologically-active atherosclerotic lesions and thrombosis.

\section{Target groups}

Target groups of the research studies included in this dissertation are 1) patients with symptomatic atherosclerotic carotid arteries, which are subjected to revascularization procedures; 2) cardiologists; 3) the community of scientists in basic research; and 4) pharmaceutical and medical industry.

The advances in knowledge presented within this thesis may be beneficial for the development of new imaging protocols and contrast agents for patients subjected to carotid artery revascularization procedures or patients with oscillatory arterial 
blood pressure and hypercholesterolemia. These new imaging approaches could be easily performed by cardiologists with general US imaging competences. Moreover, advancing low cost diagnostic methods, which could partially replace expensive diagnostic imaging approaches, such as PET, CT, and MRI, would have both health and socio-economic impact, as it decreases the financial burden on the healthcare system and simultaneously increases US's potential as a routine medical prevention modality. Additionally, this thesis reports findings and results for scientists, as well as pharmaceutical and medical industry, that may further use these innovative methods and principles as basis for future research and to develop new diagnostic and therapeutic agents.

\section{Activity/Products}

Several new findings have been developed and described in this dissertation. An ex vivo TPLSM protocol for imaging explanted murine carotid arteries has been described and implemented ${ }^{2}$ in Chapter 2. Moreover, TPLSM was combined with molecular US imaging and the combination is presented as a multiscale diagnostic approach.

Finally, different targeted MB have been generated that can be used to monitor endothelial regeneration (Chapter 2 and Chapter 4 ) or to evaluate transient endothelial activation under acute blood flow variations (Chapter 5).

\section{Innovation}

The studies included in this thesis are innovative thanks to several reasons. Multiscale molecular vascular imaging with TPLSM and US using targeted MB was successfully applied as new approach to examine endothelial dysfunction. Moreover, dual TPLSM/US molecular imaging of carotid arteries, subjected to high shear stress, can be described as novel and innovative. Additionally, the idea to use molecular US to evaluate vascular damage and repair after 
revascularization procedures, or to assess early vascular dysfunction triggered by oscillatory arterial blood pressure is as well original.

\section{Implementation}

The research results of this thesis have been implemented and shared in numerous presentations on national and international conferences, receiving international recognition and a best poster award at the 81st Congress of the European Atherosclerosis Society, Lyon 2-5 June, 2013, France.

Moreover, the research results have been published in international peerreviewed journals with high impact factor.

\section{References}

1. Celermajer DS, Chow CK, Marijon E, Anstey NM, Woo KS. Cardiovascular disease in the developing world: prevalences, patterns, and the potential of early disease detection. J Am Coll Cardiol. 2012;60(14):1207-1216.

2. Wu Z, Curaj $A$, Fokong $S$, et al. Rhodamine-loaded intercellular adhesion molecule-1-targeted microbubbles for dual-modality imaging under controlled shear stresses. Circ Cardiovasc Imaging. 2013;6(6):974-981. 



\section{List of abbreviations}

AM: Amplitude modulation

ApoE: Apolipoprotein E

a.u: Arbitrary units

CEUS: Contrast-Enhanced Ultrasound

DAPI: 4',6-diamidino-2-phenylindole

d: Days

D: Dyastole

$\mathrm{dB}$ : Decibels

EC: Endothelial cells

ECGM II: Endothelial cell growth medium

EDTA: Ethylenediaminetetraacetic acid

funcUS: Functional ultrasound

GM-CSF: Granulocyte-macrophage colony-stimulating factor

HEPES: 4-(2-hydroxyethyl)-1-piperazineethanesulfonic acid

HFD: Atherogenic diet

HUVEC: Human umbilical vein endothelial cells

Hz: Hertz

ICAM-1: Intercellular adhesion molecule -1

IFN-y: Interferon-y

IL: Interleukin

JAM-A: Junctional adhesion molecule -A 
$\mathrm{L}$ : Arterial lumen

LCA: Left carotid artery

LDL: Low density lipoprotein

LDLr: Low density lipoprotein receptor

LPS: Lipopolysaccharides

M: Macrophages

MB: Microbubbles

$\mathrm{MB}_{\mathrm{CTR}}$ : Control microbubbles

MB ${ }_{\text {ICAM-1: ICAM-1-targeted microbubbles }}$

MBJAM-A: JAM-A-targeted microbubbles

MBVCAM-1: VCAM-1-targeted microbubbles

M-CSF: macrophage-colony stimulating factor

$\mathrm{MHz}$ : Megahertz

min: Minutes

M-mode: Motion mode

molUS: Molecularly-targeted ultrasound

MRI: Magnetic resonance imaging

NA: numeric aperture

NIR: (Near) infrared

NO: Nitric oxide

PBCA: Poly(n-butyl cyanoacrylate)

PECAM-1: Platelet endothelial cell adhesion molecule -1

PET: Positron emission tomography 
PI: Pulse inversion

PL: Partial-ligation

PWD: Pulse Wave Doppler

PW-mode: Pulse wave mode

RBC: Red blood cells

RCA: Right carotid artery

S: Systole

SHG: Second harmonic generation

SM: smooth muscle

SMC: smooth muscle cells

SPECT: Single-photon emission computed tomography

THG: Third harmonic generation

TNF-a: Tumor necrosis factor $\alpha$

USPIOs: Ultra-small Iron oxide particles

VCAM-1: Vascular cell adhesion molecule -1

WI: Wire-injury

wt: Wild type

18F-FDG: 18F-fluorodeoxyglucose 



\section{Biography}

Adelina Băleanu-Curaj (married, one son and one daughter) was born on May 14, 1985 in Bucharest, Romania. She studied medicine at the "Carol Davila" University of Medicine and Pharmacy of Bucharest from 2004-2010. During her studies, she was a student in practice at Department for Cellular and Molecular Medicine - "Carol Davila“ Medical Faculty under the supervision of Dr. Mircea Leabu. Meanwhile, she was carrying out research for her Diploma thesis under the same supervision. In 2007, she did an internship in the Institute for Molecular Cardiovascular Research, RWTH Aachen University, under the supervision of Dr. Elisa Liehn. In 2014, she obtained a German PhD title (Dr. med) equivalent to an international master degree in the Institute for Experimental Molecular Imaging and Institute for Molecular Cardiovascular Research, RWTH Aachen University, under the supervision of Prof. Dr. Fabian Kiessling and Dr. Elisa Liehn. Since 2014 she started her PhD trajectory in the School for Oncology and Developmental Biology, Maastricht University, under the supervision of Prof. Dr. Frans Ramaekers and Prof. Dr. Marc van Zandvoort, and the Institute for Experimental Molecular Imaging, RWTH Aachen University, under the supervision of Prof. Dr. Fabian Kiessling. During her PhD trajectory, she was performing ex vivo and in vivo molecular ultrasound imaging in murine models for endothelial activation and consequent atherosclerotic plaque development. With this thesis, she now completes the project entitled "Molecular ultrasound imaging - a powerful tool for the diagnosis of endothelial dysfunction and arterial inflammation". With an ongoing fascination for basic research in the field of cardiovascular imaging, she continues to work as a post-doctoral researcher in the Institute for Molecular Cardiovascular Research, RWTH Aachen University. 



\section{List of publications}

Curaj A, Wu Z, van Zandvoort M, Gresch M, Weber C, Lammers T, Liehn E, Kiessling F. Molecular ultrasound imaging of JAM-A depicts acute alterations in blood flow and early endothelial dysregulation. Under revision in ATVB.

Curaj A, Wu Z, Staudt M, Liehn E. Induction of accelerated atherosclerosis in mice: the "wire-injury" model. J Vis Exp. Accepted for publication.

Curaj A, Simsekylmaz S, Staudt M, Liehn E. Minimal invasive surgical procedure of inducing myocardial infarction in mice. J Vis Exp. 2015, 4(99).

Curaj A, Wu Z, Fokong S, Liehn E, Weber C, Burlacu A, Lammers T, Kiessling $F$, van Zandvoort M, Kiessling F. Noninvasive molecular ultrasound monitoring of vessel healing after intravascular surgical procedures in a preclinical setup. ATVB 2015, 35(6), 1366-73.

Wu $Z^{*}$, Curaj $\mathbf{A}^{*}$, Fokong $\mathrm{S}$, Liehn E, Weber $\mathrm{C}$, Lammers $\mathrm{T}$, Kiessling F, van Zandvoort M. Rhodamine-loaded intercellular adhesion molecule-1-targeted microbubbles for dual-modality imaging under controlled shear stresses. Circ Cardiovasc Imaging 2013, 6, 974-81. (*equal contribution)

Fokong S, Fragoso A, Rix A, Curaj A, Wu Z, Lederle W, Iranzo O, Gätjens J, Kiessling F, Palmowski M. Ultrasound Molecular Imaging of E-Selectin in Tumor Vessels Using Poly n-Butyl Cyanoacrylate Microbubbles Covalently Coupled to a Short Targeting Peptide.Invest Radiol. 2013, 48(12):843-50.

Oral H, Kanzler I, Tuchscheerer N, Curaj A, Simsekyilmaz S, Sönmez TT, Radu E, Postea O, Weber C, Schuh A, Liehn EA. CXC chemokine KC fails to induce neutrophil infiltration and neoangiogenesis in a mouse model of myocardial infarction. J Mol Cell Cardiol. 2013, 60:1-7.

Koczera P, Wu Z, Fokong S, Theek B, Appold L, Jorge S, Möckel D, Liu Z, Curaj A, Storm G, van Zandvoort M, Kiessling F, Lammers T. Fluorescently labeled 
microbubbles for facilitating translational molecular ultrasound studies. Drug Deliv TransI Res. 2012, 2(1):56-64.

Liehn EA, Postea O, Curaj A, Marx N. Repair after myocardial infarction, between fantasy and reality the role of chemokines. J Am Coll Cardiol. 2011, 58(23):235762.

Liehn EA, Piccinini AM, Koenen RR, Soehnlein O, Adage T, Fatu R, Curaj A, Popescu A, Zernecke A, Kungl AJ, Weber C.A. New Monocyte Chemotactic Protein-1/Chemokine CC Motif Ligand-2 Competitor Limiting Neointima Formation and Myocardial Ischemia/Reperfusion Injury in Mice. J Am Coll Cardiol. 2010, 56(22):1847-57.

Rosu T, Pahontu E, Pasculescu S, Georgescu R, Stanica N, Curaj A, Popescu $A$, Leabu M. Synthesis and characterization of novel $\mathrm{Cu}(\mathrm{II})$ and $\mathrm{Pd}(\mathrm{II})$ complexes with 2-hydroxy-8-R-tricyclo[7.3.1.0.(2,7)]tridecane-13-one thiosemicarbazone. Study on biological activity. Eur J Med Chem. 2010, 45(4):1627-34. 
List of publications 



\section{Acknowledgements}

I cannot believe that I am now writing the acknowledgements of my $\mathrm{PhD}$ thesis. It has been a difficult jurney, but with the support of my supervisors, mentors, colleagues, friends and family, everything was finally possible. Therefore, I would like to express my sincere gratitude to everyone who brought contribution in completing my PhD trajectory.

First, I would like to thank Prof. Dr. Frans Ramaekers, my first supervisor, for allowing me to complete my $\mathrm{PhD}$ trajectory under his supervision. Starting with the first talk about the $\mathrm{PhD}$ trajectory I had a positive felling that our collaboration will be successful. Several things I certainly learned from you: 1) trust people and offer them chances; 2 ) rely on your team members.

Furthermore, I am very grateful to Prof. Dr. Marc van Zandvoort for all his support. Dear Marc, you played a major role in making my PhD trajectory possible. Since the first day we met, I knew that such a possitive person can only be a motivator. I did not only developed professionally by being part of the 2-photon team, but I aslo lerned from you to value personal things in life.

I would like to express my special appreciation and thanks to Prof. Dr. Fabian Kießling, for being my mentor and supervisor. I always felt your support. With every discussion we had, I felt pushed one step further into completing my goal. Under your close supervision, I have developed myself as a scientist. Thank you for all your support and for trusting me. Moreover, thank you for all your pieces of advice. Many of them were very important for me, in making life decisions.

Likewise, I am very grateful to Priv.-Doz. Dr. Elisa Liehn, from which I have learned how to make science with passion. She was always beside me to teach me and to guide me. She offered her support not only professionally but also in my private life. Thanks to the discussions we had, I took some important decisions in my private and professional life. Dear Elisa, thank you so much for always being there to motivate me. 
I would like to thank one of my best friends, Dr. Wu Zhuojun. Dear Wu, you are trouly a wonderful person to work with. I have learned so many things from you, and I think you had much contribution in my professional development. Thank you for being patient and for trusting me. I hope we will sonn be a team again.

Next, I would like to thank my friends Roya Soltan for all her support in the lab, and all her love; Santosh Vijaian for all the motivatory talkes we had, and for the nice moments we have spent together; Baixue Zhou for shearing life experience in Lyon and not only, and for all the fun we had by traveling together; Mrs. Anni Mayer for her enourmous care; Mareike Staudt for her support in the lab and German lessons.

I would like to thank my aunt, Stefania lancu for her support when I needed time to concentrate on writing the thesis. As well, I would like to thank my friend, Cosmin Holeab for his assistance in finalizing such a wonderful cover for my thesis.

Furthermore I would like to thank my family members for their unconditional love, and balance in my life. My dad, Adrian Curaj, who always supported and guided me in my carrier and to whom I owe the decision of starting my $\mathrm{PhD}$ abroad. My mom, Aurelia Curaj, who was always carrying and supporting me, trying to keep her feelings away from me, not to distract me from the target. My sister, Alice, my broder-in-low, Paul, and my nephew,Tudor, for being next to me all these years.

My father-in-low, Stelian Băleanu, and Daniela Cristescu, for all their love and support. Together, we have passed two mile-stones in my life. Thank you for your enormous care.

My mother-in-low, loana Stancu, for all her love and support.

At last, but not least, I thank my husband Bogdan, for his support, pieces of advice, and all his love; and my kids, Philip and Emma, who are the most patient and wonderful kids. You were next to me day and night. Without you three, I would have not make it. You are fantastic! 
